TRANSACTIONS OF THE

AMERICAN MATHEMATICAL SOCIETY

Volume 358, Number 7 , Pages 3169-3206

S 0002-9947(06)03834-7

Article electronically published on February 20, 2006

\title{
LAYERS AND SPIKES IN NON-HOMOGENEOUS BISTABLE REACTION-DIFFUSION EQUATIONS
}

\author{
SHANGBING AI, XINFU CHEN, AND STUART P. HASTINGS
}

\begin{abstract}
We study $\varepsilon^{2} \ddot{u}=f(u, x)=A u(1-u)(\phi-u)$, where $A=A(u, x)>$ $0, \phi=\phi(x) \in(0,1)$, and $\varepsilon>0$ is sufficiently small, on an interval $[0, L]$ with boundary conditions $\dot{u}=0$ at $x=0, L$. All solutions with an $\varepsilon$ independent number of oscillations are analyzed. Existence of complicated patterns of layers and spikes is proved, and their Morse index is determined. It is observed that the results extend to $f=A(u, x)\left(u-\phi_{-}\right)(u-\phi)\left(u-\phi_{+}\right)$with $\phi_{-}(x)<$ $\phi(x)<\phi_{+}(x)$ and also to an infinite interval.
\end{abstract}

\section{INTRODUCTION}

In this paper we are concerned with the existence and stability of equilibrium solutions for reaction diffusion equations of the form

$$
u_{t}=\varepsilon^{2} u_{x x}-f(u, x)
$$

on an interval $(a, b)$ with Neumann boundary conditions $u_{x}(t, a)=u_{x}(t, b)=0$. Such problems have a considerable history since they arise in a variety of physical contexts, as can be seen by consulting the citations in [FR2. An early mathematical reference is the paper AMPP, which studied all of the stable solutions in the case where $f=u(1-u)(\phi-u)$. Subsequent results on "single layer" unstable solutions were obtained in $[\mathrm{HS}]$. In $[\mathrm{AH}]$ the problem was studied with $f(u, x)=$ $u^{3}-\lambda u+\cos x, \lambda>3 / \sqrt[3]{4} ; \mathrm{AH}$ includes some of the conclusions on existence in this paper for the special case considered there. However here we consider a more general nonlinearity, obtaining an even richer collection of solutions since the "multiple spikes" found below are not present for the model in $\mathrm{AH}$. Similar results were studied in $\mathrm{A}$ and $\mathrm{HM}$, although in both of these cases the nonlinearities were quadratic rather than cubic in nature. Other related papers include [CP], FR1], HASL, HSG], $\mathrm{KU}$ ] and [RO].

The main focus of this paper, however, is the Morse index of unstable solutions. An early related result is in $\overline{\mathrm{ABF}}$, where solutions with arbitrary given Morse index are shown to exist for arbitrarily small $\epsilon$. Here, after constructing layer and spike type solutions by a shooting method, we are able to determine all of their Morse indices. The first paper to do this for models of this kind was by Nakashima [N], who considered the "balanced" case $f=A(x) u(1-u)\left(\frac{1}{2}-u\right)$. Her methods were variational (and so very different from ours). Our results do not apply to her model.

Received by the editors April 17, 2002 and, in revised form, September 1, 2004.

2000 Mathematics Subject Classification. Primary 35K57.

The second author thanks the National Science Foundation Grant DMS-0203991 for their support. 
Subsequently she again used variational methods to study layers in the unbalanced case, obtaining results similar to some of ours (Nakashima, private communication). As far as we know, variational methods have not been successfully applied to study the Morse index of spike solutions. We mention as well that many of the results in our paper were independently conjectured by Matano (private communication). We will see below that the computations needed to determine the Morse indices of layers and spikes in this problem are quite delicate.

To obtain steady state solutions, we consider the boundary value problem

$$
\left\{\begin{array}{l}
\varepsilon^{2} \ddot{u}=f(u, x), \quad x \in \mathrm{I}:=(a, b), \\
\dot{u}(a)=\dot{u}(b)=0,
\end{array}\right.
$$

for a sufficiently small positive parameter $\varepsilon$, where ${ }^{\cdot}=\frac{d}{d x}$ and $f=\frac{\partial F}{\partial u}=: F_{u}$ is the derivative of a smooth bistable potential $F$. More precisely,

$$
\left\{\begin{array}{c}
\{u \in \mathbb{R} \mid f(u, x)=0\}=\{0, \phi(x), 1\} \quad \forall x \in \overline{\mathrm{I}}, \\
0<\phi(x)<1, \quad f_{u}(0, x)>0, \quad f_{u}(1, x)>0 \quad \forall x \in \overline{\mathrm{I}} .
\end{array}\right.
$$

At the end of the paper we will show that a transformation takes

$$
f=A(u, x)\left[u-\phi_{-}(x)\right][u-\phi(x)]\left[u-\phi_{+}(x)\right],
$$

where $A>0$ and $\phi_{-}<\phi<\phi_{+}$, to a slight extension of the standard form (2), the difference being that the new function $f$ depends as well on $\varepsilon$. Our results extend to the type of functions obtained by such a transformation.

Solutions to (11) correspond to critical points of the energy functional

$$
\mathcal{E}_{\varepsilon}(u)=\int_{a}^{b}\left\{\frac{\varepsilon}{2} u_{x}^{2}(x)+\frac{1}{\varepsilon} F(u(x), x)\right\} d x=\int_{\frac{(a-\xi)}{\varepsilon}}^{\frac{(b-\xi)}{\varepsilon}}\left[\frac{1}{2} U_{y}^{2}(y)+F(U(y), \xi+\varepsilon y)\right] d y,
$$

where $U(y):=u(\xi+\varepsilon y)$ and $\xi \in(a, b)$. We are only interested in solutions that have an $\varepsilon$ independent bounded number of oscillations in any bounded interval, namely, solutions in the set

$\mathcal{S}_{N, \varepsilon}=\{u \mid u$ solves (10), $\dot{u}=0$ has at most $N$ roots in any interval of length $\leq 1\}$.

With such a definition, we can allow the length $b-a$ to be arbitrarily large.

To study solutions in $\mathcal{S}_{N, \varepsilon}$, we introduce

$$
\begin{gathered}
J(x):=\int_{0}^{1} f(s, x) d s, \quad \phi_{0}(x):= \begin{cases}0 & \text { if } J(x)<0, \\
1 & \text { if } J(x)>0,\end{cases} \\
F(u, x):=\int_{\phi_{0}(x)}^{u} f(s, x) d s, \quad \phi^{*}(x) \in[0,1] \backslash\left\{\phi_{0}(x)\right\}: F\left(\phi^{*}, x\right)=0, \\
S(x):=\frac{d}{d x} \int_{\phi_{0}(x)}^{\phi^{*}(x)} \sqrt{2 F(s, x)} d s=\int_{\phi_{0}}^{\phi^{*}} \frac{F_{x}}{\sqrt{2 F}} d s \quad \text { if } J(x) \neq 0 .
\end{gathered}
$$

Note that $\phi$ lies between $\phi_{0}$ and $\phi^{*}$ and $F(s, x)>0$ for all $s$ between $\phi_{0}$ and $\phi^{*}$.

As $\varepsilon$ is small, near each $x_{0} \in \mathbf{I}$, any solution to (1) can be approximated by $U\left(\left(x-x_{0}\right) / \varepsilon\right)$, where

$$
U^{\prime \prime}(y)=f\left(U(y), x_{0}\right) \quad \forall y \in \mathbb{R} .
$$

Note that $U \equiv 0$ and $U \equiv 1$ are the only stable constant solutions. Physically they correspond to two phases, which we call 0 and 1 . When $J\left(x_{0}\right) \neq 0$, the 
potential energy density $F\left(1, x_{0}\right)$ and $F\left(0, x_{0}\right)$ of the two phases are different. In our definition, $\phi_{0}\left(x_{0}\right)$ is the non-minimal potential energy phase, whereas the other $\left(0\right.$ if $\phi_{0}=1$ and 1 if $\left.\phi_{0}=0\right)$ is the minimal energy phase.

On the phase plane, all the trajectories are given by $U^{\prime 2}-2 F\left(U, x_{0}\right)=C$. Since we are only interested in solutions to (1) that have finitely many (independent of $\varepsilon$ ) oscillations, only the trajectories corresponding to $C=0$ will be relevant (cf. Lemma 2.1 below).

If $J\left(x_{0}\right)=0$, the trajectory $U^{\prime 2}=2 F\left(U, x_{0}\right)$ gives a heteroclinic orbit connecting 0 and 1 , which will be called a (phase transition) layer or an interface. The interfacial energy associated with a layer is

$$
\mathcal{E}\left(x_{0}\right) \equiv \int_{-\infty}^{\infty}\left[\frac{1}{2} U^{\prime 2}(y)+F\left(U(y), x_{0}\right)\right] d y=\left|\int_{\phi_{0}\left(x_{0}\right)}^{\phi^{*}\left(x_{0}\right)} \sqrt{2 F\left(s, x_{0}\right)} d s\right| .
$$

To study layered solutions to (11), we will assume that all roots of $J=0$ are nondegenerate, namely,

$$
J^{2}(x)+\dot{J}(x)^{2}>0 \quad \forall x \in \overline{\mathrm{I}} .
$$

Suppose that $J\left(x_{0}\right)=0>\dot{J}\left(x_{0}\right)$. Then the minimal potential energy phase switches from 0 to 1 as $x$ passes the position $x_{0}$. Hence if $U(-\infty)=0$ and $U(\infty)=1$, the phase transition is from the minimal energy phase before $x_{0}$ to the minimal energy phase after $x_{0}$. On the other hand, if $U(-\infty)=1$ and $U(\infty)=0$, the transition is from the non-minimal energy phase to the non-minimal energy phase.

When $J\left(x_{0}\right) \neq 0$, the trajectory $U^{\prime 2}=2 F\left(U, x_{0}\right)$ gives a homoclinic orbit which we will call a spike, with base $\phi_{0}\left(x_{0}\right)$ and peak $\phi^{*}\left(x_{0}\right)$. Regarding a spike as two layers, the excess energy a spike adds to a ground constant state $u=\phi_{0}(x)$ is then twice $\mathcal{E}\left(x_{0}\right)$, defined in (3). It turns out that spikes can only appear near those points where $S=0$, e.g. near the critical point of $\mathcal{E}(x)$ or near the boundaries. To study these solutions, we assume that zeros of $S$ are non-degenerate, i.e.,

$$
S^{2}(x)+\dot{S}^{2}(x)>0 \quad \forall x \in\{z \in \overline{\mathrm{I}} \mid J(z) \neq 0\} .
$$

For an illustration of the functions $J$ and $S$, one can calculate that when $f=$ $u(u-1)(u-\phi)$,

$$
J(x)=\frac{1}{6}\left(\phi-\frac{1}{2}\right), \quad S \propto \dot{\phi} .
$$

Hence, for this particular non-linearity, the set where $S=0$ is the set of all critical points of $\phi$.

Any solution to (11) is necessarily bounded between 0 and 1 . Hence, it is concave in the set $\{u>\phi\}$ and convex in $\{u<\phi\}$. An oscillation will then be referred to as a root to $u=\phi$.

This paper is divided into three parts.

In Part I, we investigate the location of oscillations of solutions in $\mathcal{S}_{N, \varepsilon}$. We show that oscillations are either layers or spikes. Layers appear only near $Z_{J}$ and spikes only near $Z_{S}$ where

$$
Z_{J}=\{z \mid J(z)=0\}, \quad Z_{S}:=\left\{z \notin Z_{J} \mid(z-a)(z-b) S(z)=0\right\} .
$$

Multiple layers can exist, but only in transitions from a non-minimal potential energy phase to a non-minimal energy phase (i.e., from $\phi_{0}\left(x_{0}-\right)$ to $\phi_{0}\left(x_{0}+\right)$ ). Multiple spikes can exist, but only at those interior points where $S=0<J \dot{S}$, or 
at the boundary point a if $\{J(a) S(a)>0$ or $S(a)=0<J(a) \dot{S}(a)\}$, or at $b$ if $\{J(b) S(b)<0$ or $S(b)=0<J(b) \dot{S}(b)\}$.

In Part II, we show that there exist solutions with arbitrarily prescribed numbers of oscillations at places allowed by the italicized sentences in the previous paragraph.

In Part III, we study the dimension of the unstable manifold, i.e., the Morse index, of an arbitrary solution in $\mathcal{S}_{N, \varepsilon}$. We show that the Morse index is equal to $n-n_{s l}-n_{s s}$, where $n$ is the total number of oscillations, $n_{s l}$ is the number of minimal energy phase to minimal energy phase transitions, and $n_{s s}$ is the number of interior spikes near which $J \dot{S}<0$.

Our analysis would have been much simpler if boundary oscillations were not addressed. For simplicity, the reader can skip the analysis for boundary oscillations.

Throughout this paper, we always assume (2), (4), and (5). For simplicity, we consider only the case that $a, b$, and $f$ are independent of $\varepsilon$. Nevertheless, when $a, b$, and $f$ depend on $\varepsilon$, all the proofs remain unchanged, provided that $f$ is uniformly smooth, and that $\phi, 1-\phi, S^{2}+\dot{S}^{2}$ and $J^{2}+\dot{J}^{2}$ are uniformly positive. For boundary layers or spikes, we need to assume that either $a \in Z:=\{z \in \mathbb{R} \mid J(z) S(z)=0\}$ or $\operatorname{dist}(a, Z) \geq \sqrt{\varepsilon}$, and either $b \in Z$ or $\operatorname{dist}(b, Z) \geq \sqrt{\varepsilon}$.

Finally, we remark that assumption (2) implies, for all $x \in \overline{\mathrm{I}}$, the following:

$$
\begin{gathered}
\left|f(s, x), \quad f_{x}(s, x), \quad f_{x x}(s, x)\right| \leq K s(1-s) \quad \forall s \in[0,1], \\
\left|G(s, \hat{s}, x), \quad G_{x}(s, \hat{s}, x), \quad G_{x x}(s, \hat{s}, x)\right| \\
\leq K \begin{cases}s^{2}-\hat{s}^{2} & \text { if } 0 \leq \hat{s}<s \leq \phi(x), \\
(1-s)^{2}-(1-\hat{s})^{2} & \text { if } 1 \geq \hat{s} \geq s \geq \phi(x),\end{cases} \\
G(s, \hat{s}, x) \geq \frac{1}{K} \begin{cases}s^{2}-\hat{s}^{2} & \text { if } \phi(x) \geq s \geq \hat{s} \in\left[0, \frac{1}{2} \phi\right), \\
(1-s)^{2}-(1-\hat{s})^{2} & \text { if } \phi(x) \leq s \leq \hat{s} \in\left(\frac{1}{2}(1+\phi), 1\right],\end{cases}
\end{gathered}
$$

where $K$ is a positive constant and

$$
G(s, \hat{s}, x):=F(s, x)-F(\hat{s}, x)=\int_{\hat{s}}^{s} f(v, x) d v .
$$

\section{Part I: Solutions in $\mathcal{S}_{N, \varepsilon}$}

In this part we study a general solution in $\mathcal{S}_{N, \varepsilon}$. When needed, the following even (about $a$ and $b$ ) and periodic (with period 2(b-a)) extensions are implicitly assumed:

$$
\begin{aligned}
f(s, a+y) & =f(s, a-y), \quad f(s, x)=f(s, 2(b-a)+x), \quad \forall y \in[a-b, 0], x \in \mathbb{R} . \\
u(a+y) & =u(a-y), \quad u(x)=u(2(b-a)+x)
\end{aligned}
$$

With such an extension, $f$ is continuous and piecewise smooth, and $u$ solves $\varepsilon^{2} \ddot{u}=f$ on $\mathbb{R}$.

\section{LOCATIONS OF OSCILLATIONS}

Lemma 2.1. Let $N \geq 1$ be a given integer. Then

$$
\lim _{\varepsilon \searrow 0} \sup _{u \in \mathcal{S}_{N, \varepsilon}} \max _{x \in \overline{\mathrm{I}}}\left|\left[\varepsilon^{2} \dot{u}^{2}-2 F(u, x)\right] u(1-u)\right|=0 .
$$


Proof. By a maximum principle, if $u \not \equiv 0$ and $u \not \equiv 1$, then $0<u<1$ in $\overline{\mathrm{I}}$.

Suppose that the assertion is not true. Then there exist $\delta>0$ and a sequence $\left\{\varepsilon_{i}, u^{i}, x_{i}\right\}_{i=1}^{\infty}$ such that $\lim _{i \rightarrow \infty} \varepsilon_{i}=0$ and for each $i \geq 1$, there holds $\varepsilon_{i}>0$, $u^{i} \in \mathcal{S}_{N, \varepsilon_{i}}$, and $\left.\left|\varepsilon_{i}^{2} \dot{u}^{i 2}-F\left(u^{i}, x_{i}\right)\right| u^{i}\left(1-u^{i}\right)\right|_{x=x_{i}} \geq \delta$. By taking a subsequence if necessary, we can assume that $\lim _{i \rightarrow \infty} x_{i}=x_{*} \in \overline{\mathrm{I}}$.

Define $U_{i}(y)=u^{i}\left(x_{i}+\varepsilon y\right)$ for $y \in\left[-(b-a) / \varepsilon_{i},(b-a) / \varepsilon_{i}\right]$. Then $U_{i}^{\prime \prime}=$ $f\left(U_{i}, x_{i}+\varepsilon y\right)\left({ }^{\prime}:=\frac{d}{d y}\right)$. Hence $\left\{U_{i}\right\}_{i=1}^{\infty}$ is a bounded family in $C^{2}$, and, by taking a subsequence if necessary, there exists $U \in C^{2}(\mathbb{R})$ such that $\lim _{i \rightarrow \infty}\left(U_{i}, U_{i}^{\prime}\right)=$ $\left(U, U^{\prime}\right)$ uniformly in any compact subset of $\mathbb{R}$. In addition $U^{\prime \prime}=f\left(U, x_{*}\right)$, so that $U^{\prime 2}=2 F\left(U, x_{*}\right)+C$ for some $C$. One notes that if $C>0$, then the solution is unbounded on $\mathbb{R}$, and if $C<0$, then the solution is periodic. But both are impossible, since $U_{i}$ is bounded and can have at most $2 N+1$ oscillations in $\left[-1 / \varepsilon_{i}, 1 / \varepsilon_{i}\right]$. Hence, we must have $C=0$. But this implies that $\lim _{i \rightarrow \infty}\left|\varepsilon_{i}^{2} \dot{u}^{i 2}\left(x_{i}\right)-F\left(u^{i}, x_{i}\right)\right|=\lim _{i \rightarrow \infty}\left|U_{i}^{\prime 2}(0)-F\left(U_{i}(0), x_{i}\right)\right|=0$. This completes the proof.

We remark that if $b-a$ is not bounded, then the subsequence is taken such that $\lim _{i \rightarrow \infty} F\left(\cdot, x_{i}\right)=F^{*}(\cdot)$ for some smooth bistable potential $F^{*}$.

Lemma 2.2. For each integer $N \geq 1$, there exists $\varepsilon(N)>0$ such that for each $u \in \mathcal{S}_{N, \varepsilon}$ with $\varepsilon \in(0, \varepsilon(N)]$, there are an integer $n \geq 1$ and points $\tau_{0}, \cdots, \tau_{n}$ and $z_{1 / 2}, \cdots, z_{n-1 / 2}$ such that

$$
\begin{gathered}
\{x \in \overline{\mathrm{I}} \mid \dot{u}(x)=0\}=\left\{\tau_{0}, \cdots, \tau_{n}\right\}, \\
\{x \in \overline{\mathrm{I}} \mid u(x)=\phi(x)\}=\left\{z_{1 / 2}, \cdots, z_{n-1 / 2}\right\}, \\
a=\tau_{0}<z_{1 / 2}<\tau_{1}<\cdots<z_{n-1 / 2}<\tau_{n}=b .
\end{gathered}
$$

Proof. If $u(z)=\phi(z)$, then $\varepsilon^{2} \dot{u}^{2} \approx 2 F(\phi, z)>0$, so that $\left.\frac{d}{d x}(u-\phi)\right|_{x=z} \neq 0$. Hence the number of roots of $u(x)=\phi(x)$ is finite. In addition, between each pair of neighboring roots of $u=\phi, \varepsilon^{2} \ddot{u}=f \neq 0$, so $u$ is either convex or concave, and hence there is a unique local extreme, i.e., a unique $\tau$ such that $\dot{u}(\tau)=0$. The assertion of the lemma thus follows.

In the sequel, $N \geq 1$ is a fixed integer and $u \in \mathcal{S}_{N, \varepsilon}$ with $\varepsilon$ sufficiently small. We use the notation in Lemma 2.2. With the even and periodic extension, $\tau_{i}, z_{i+1 / 2}$ are well defined for all integers $i$.

Definition 1. Let $l$ and $k$ be integers satisfying $-N \leq l<n, 0<k \leq n+N$, $0<k-l \leq 2 N$.

We call $\left(\tau_{l}, \tau_{k}\right)$ an interval with a cluster of oscillations if

$z_{k-1 / 2}-z_{l+1 / 2} \leq(k-l-1) \sqrt{\varepsilon}, \quad z_{l-1 / 2}+\sqrt{\varepsilon}<z_{l+1 / 2}, \quad z_{k-1 / 2}+\sqrt{\varepsilon}<z_{k+1 / 2}$.

A cluster is referred to as interior if $0 \leq l<k \leq n$. Otherwise it is called a boundary cluster.

An interior cluster is called a cluster of spikes if $k-l$ is even, and layers if $k-l$ is odd.

A boundary cluster is called a boundary cluster of layers if $J=0$ at the boundary point; otherwise it is called a boundary cluster of spikes.

A cluster of spikes is called multiple if $k-l \geq 4$ and single if $k-l=2$. Similarly, a cluster of layers is called multiple if $k-l \geq 2$ and single if $k-l=1$. 
Note that for boundary clusters, either $k+l=0$ or $k+l=2 n$, so that $k-l$ is always even. Consequently, boundary layers are always multiple. Restricted to the interval $[a, b]$, a single boundary spike will be called a half boundary spike. (In this terminology, a half boundary layer exists only for Dirichlet boundary conditions.)

If we define an equivalence relation $z_{i+1 / 2} \sim z_{j+1 / 2}$ if $\left|z_{i+1 / 2}-z_{j-1 / 2}\right| \leq|i-j| \sqrt{\varepsilon}$, then each equivalence class $\left\{z_{i+1 / 2}\right\}_{i=l}^{k-1}$ can be regarded as a cluster, and belongs to an interval $\left(\tau_{l}, \tau_{k}\right)$ with a cluster. In this manner, the decomposition is unique. Also, each cluster is either of spikes or of transition layers.

Theorem 1. Assume that $f$ satisfies (21), (4) and (5), that $N$ is a fixed positive integer and $\varepsilon>0$ is sufficiently small. Define $Z_{J}$ and $Z_{S}$ as in (6).

Let $u \in \mathcal{S}_{N, \varepsilon}$ be arbitrary and let $\left(\tau_{l}, \tau_{k}\right)$ be a cluster of oscillation of $u$. Then there exists $z^{*} \in Z_{J} \cup Z_{S}$ such that $z_{i+1 / 2}=z^{*}+O(\varepsilon|\ln \varepsilon|)$ for all $i=l, \cdots, k-1$. In addition:

(i) if $k-l=1$, then $z^{*} \in(a, b)$ and $J\left(z^{*}\right)=0$;

(ii) if $k-l>1$, then $u\left(\tau_{l}\right)=\phi_{0}\left(z^{*}-\right)+O\left(e^{-1 / \sqrt{\varepsilon}}\right)$ and $u\left(\tau_{k}\right)=\phi_{0}\left(z^{*}+\right)+$ $O\left(e^{-1 / \sqrt{\varepsilon}}\right)$;

(iii) if $k-l>2$, then $J S>0$ in $\left(z^{*}, z^{*}+\sqrt{\varepsilon}\right)$ and $J S<0$ in $\left(z^{*}-\sqrt{\varepsilon}, z^{*}\right)$.

Remark 2.1. (1) If $z^{*} \in(a, b) \cap Z_{J}$, then $\phi_{0}\left(z^{*}-\right) \neq \phi_{0}\left(z^{*}+\right)$, so that $k-l$ is odd. On the other hand, if $z^{*} \in Z_{S} \cup\{a, b\}$, then $\phi_{0}\left(z^{*}+\right)=\phi_{0}\left(z^{*}-\right)$, so that $k-l$ is even. Hence, transition layers appear only near $Z_{J}$ and spikes only near $Z_{S}$.

(2) If $z^{*}=a$, then $l=-k$, and if $z^{*}=b$, then $k=2 n-l$.

(3) From (ii), multiple layers exist only in transitions from non-minimal phase to non-minimal phase.

(4) From (iii), multiple spikes exist only near those $z^{*} \in[a, b]$ at which $S\left(z^{*}\right)=$ $0<J\left(z^{*}\right) \dot{S}\left(z^{*}\right)$, or $z^{*}=a$ and $J S>0$ in $(a, a+\sqrt{\varepsilon})$, or $z^{*}=b$ and $J S<0$ in $(b-\sqrt{\varepsilon}, b)$. (Note that $J(\cdot)$ is even and $S(\cdot)$ is odd.)

(5) The definition of $S$ implies that $\lim _{x \rightarrow \xi} S(x)=\infty$ if $J(\xi)=0<\dot{J}(\xi)$ and $\lim _{x \rightarrow \xi} S(x)=-\infty$ if $J(\xi)=0>\dot{J}(\xi)$. Hence, for some positive $\delta$ depending only on $f$,

$$
\text { if } J\left(z^{*}\right)=0 \text {, then } J S<0 \text { in }\left[z^{*}-2 \delta, z^{*}\right) \text { and } J S>0 \text { in }\left(z^{*}, z^{*}+2 \delta\right] .
$$

Later on, we shall provide accurate estimates on the location of oscillations, as well as the potential energy density at points of local minimum and maximum. The following lemma plays a key role in our estimates. We use the notation

$$
\begin{aligned}
& u_{i}=u\left(\tau_{i}\right), \quad f_{i}=f\left(u_{i}, \tau_{i}\right), \quad \phi_{i}=\phi\left(\tau_{i}\right), \quad \phi_{i}^{*}=\phi^{*}\left(\tau_{i}\right), \quad \dot{u}_{i+1 / 2}=\dot{u}\left(z_{i+1 / 2}\right), \\
& \alpha(x)= \begin{cases}\sqrt{f_{u}(1, x)} & \text { if } u(x)>\phi(x), \\
\sqrt{f_{u}(0, x)} & \text { if } u(x)<\phi(x),\end{cases} \\
& E_{i}= \begin{cases}\int_{0}^{u_{i}} f\left(s, \tau_{i}\right) d s & \text { if } u_{i}<\phi_{i}, \\
\int_{1}^{u_{i}} f\left(s, \tau_{i}\right) d s & \text { if } u_{i}>\phi_{i},\end{cases} \\
& \alpha_{i}=\alpha\left(\tau_{i}\right), \quad F_{i}=F\left(u_{i}, \tau_{i}\right) .
\end{aligned}
$$

Lemma 2.3. For each $i=0, \cdots, n$,

$$
\begin{aligned}
\varepsilon^{2} \dot{u}^{2}(x) & =[2+O(\varepsilon)] G\left(u(x), u_{i}, x\right) \quad \forall x \in\left[z_{i-1 / 2}, z_{i+1 / 2}\right], \\
\varepsilon\left|\ln f_{i}^{2}\right| & =2 \alpha_{i}\left|\tau_{i}-z_{i \pm 1 / 2}\right|+O\left(\varepsilon+\left(\tau_{i}-z_{i \pm 1 / 2}\right)^{2}\right), \\
E_{i}-E_{i+1} & =J\left(z_{i+1 / 2}\right) \mathrm{H}\left(\dot{u}_{i+1 / 2}\right)+O(\varepsilon),
\end{aligned}
$$


where $\mathrm{H}(\cdot)$ is the signature function, i.e., $\mathrm{H}(s)=1$ for $s>0,=-1$ for $s<0$ and $=0$ for $s=0$.

Proof. By symmetry, we need only consider the case where $u_{i}$ is a local minimum. Then $\dot{u}>0$ and $f>0$ in $\left(\tau_{i}, z_{i+1 / 2}\right)$. Set $\nu:=\min _{x \in \mathrm{I}, s \in(0, \phi / 2]}\left\{\frac{f(s, x)}{s}\right\}>0$. Then $\varepsilon^{2} \ddot{u} \dot{u}=f \dot{u} \geq \nu u \dot{u} \chi_{\{u<\phi / 2\}}$ in $\left[\tau_{i}, z_{i+1 / 2}\right]$. After an integration, we then obtain, for some positive constant $c_{0}$ independent of $\varepsilon$,

$$
\varepsilon^{2} \dot{u}^{2}(x) \geq c_{0}\left(u^{2}(x)-u_{i}^{2}\right) \quad \forall x \in\left[\tau_{i}, z_{i+1 / 2}\right] .
$$

Now integrating $\frac{d}{d t}\left\{\frac{1}{2} \varepsilon^{2} \dot{u}^{2}(t)-G\left(u(t), u_{i}, t\right)\right\}=-G_{x}\left(u(t), u_{i}, t\right)$ and using $d t=$ $\frac{O(\varepsilon)}{\sqrt{u^{2}-u_{i}^{2}}} d u$ and $\left|G_{x}\left(u, u_{i}, t\right)\right|=O(1)\left(u^{2}-u_{i}^{2}\right)$, we obtain

$$
\begin{aligned}
& \frac{1}{2} \varepsilon^{2} \dot{u}^{2}(x)-G\left(u(x), u_{i}, x\right)=-\int_{\tau_{i}}^{x} G_{x}\left(u, u_{i}, t\right) d t \\
= & O(\varepsilon) \int_{u_{i}}^{u(x)} \frac{s^{2}-u_{i}^{2}}{\left(s^{2}-u_{i}^{2}\right)^{1 / 2}} d s=O(\varepsilon)\left(u^{2}-u_{i}^{2}\right)=O(\varepsilon) G\left(u, u_{i}, x\right),
\end{aligned}
$$

from which (8) follows.

Note that $2 G\left(u, u_{i}, x\right)=\left[2+O\left(x-\tau_{i}\right)\right] G\left(u, u_{i}, \tau_{i}\right)$. Integrating $d x=\frac{\varepsilon}{\sqrt{\varepsilon^{2} \dot{u}^{2}}}|d u|$ gives

$\varepsilon \int_{u_{i}}^{u(x)} \frac{1}{\sqrt{2 G\left(s, u_{i}, \tau_{i}\right)}} d s=\int_{\tau_{i}}^{x}[1+O(\varepsilon)]\left[1+O\left(x-\tau_{i}\right)\right] d x=x-\tau_{i}+O\left(\varepsilon+\left(x-\tau_{i}\right)^{2}\right)$.

Since $2 G\left(u, u_{i}, \tau_{i}\right)=f_{u}\left(0, \tau_{i}\right)\left(u^{2}-u_{i}^{2}\right)(1+O(u))$ and $\alpha_{i}=\sqrt{f_{u}\left(0, \tau_{i}\right)}$, we have, at $x=z_{i+1 / 2}$,

$$
\begin{aligned}
\int_{u_{i}}^{u_{i+1 / 2}} \frac{1}{\sqrt{2 G\left(s, u_{i}, \tau_{i}\right)}} d s & =\int_{u_{i}}^{\phi_{i+1 / 2}} \frac{1+O(s)}{\alpha_{i}\left(s^{2}-u_{i}^{2}\right)^{1 / 2}} d s \\
& =-\frac{\ln u_{i}}{\alpha_{i}}+O(1)=-\frac{\ln f_{i}^{2}}{2 \alpha_{i}}+O(1)
\end{aligned}
$$

since $f_{i}^{2}=\alpha_{i}^{4} u_{i}^{2}\left[1+O\left(u_{i}\right)\right]$. This gives (9). It remains to prove (10).

Integrating $\frac{d}{d t}\left(\frac{1}{2} \varepsilon^{2} \dot{u}^{2}(t)-G(u(t), 0, t)\right)=-G_{x}(u, 0, t)=O\left(u^{2}\right)$ over $t \in\left[\tau_{i}, z_{i+1 / 2}\right]$ gives

$$
\begin{aligned}
\frac{1}{2} \varepsilon^{2} \dot{u}_{i+1 / 2}^{2}+E_{i}-G\left(u_{i+1 / 2}, 0, z_{i+1 / 2}\right) & =-\int_{\tau_{i}}^{z_{i+1 / 2}} G_{x} d t \\
& =\int_{u_{i}}^{u_{i+1 / 2}} \frac{O(\varepsilon) s^{2}}{\left(s^{2}-u_{i}^{2}\right)^{1 / 2}} d s=O(\varepsilon) .
\end{aligned}
$$

Similarly, integrating $\frac{d}{d t}\left(\frac{1}{2} \varepsilon^{2} \dot{u}^{2}(t)-G(u(t), 1, t)\right)$ over $\left[z_{i+1 / 2}, \tau_{i+1}\right]$ gives us

$$
\frac{1}{2} \varepsilon^{2} \dot{u}_{i+1 / 2}^{2}+E_{i+1}-G\left(u_{i+1 / 2}, 1, z_{i+1 / 2}\right)=O(\varepsilon) .
$$

Hence, taking the difference, we obtain

$E_{i}-E_{i+1}=O(\varepsilon)+G\left(u_{i+1 / 2}, 0, z_{i+1 / 2}\right)-G\left(u_{i+1 / 2}, 1, z_{i+1 / 2}\right)=O(\varepsilon)+J\left(z_{i+1 / 2}\right)$.

This completes the proof.

From formula (9), we immediately obtain the following:

Corollary 2.4. There exists a positive constant $\mu$ that is independent of $\varepsilon$ such that if $\left(\tau_{l}, \tau_{k}\right)$ is an interval with a cluster, then $\left|f_{l}\right|+\left|f_{k}\right| \leq e^{-\mu / \sqrt{\varepsilon}}$. 
For simplicity, in the sequel, we assume $\mu=1$; otherwise, we modify the definition of clusters by replacing $\sqrt{\varepsilon}$ with $\sqrt{\varepsilon} / \mu$.

\section{TRANSITION LAYERS}

Let $z^{*} \in Z_{J}$ be fixed. In this section, we consider a generic cluster of oscillations near $z^{*}$; namely,

$$
\left[z_{l+1 / 2}, z_{k-1 / 2}\right] \in\left[z^{*}-2 \delta, z^{*}+2 \delta\right], \quad z_{k-1 / 2}<z_{k+1 / 2}-\sqrt{\varepsilon}, \quad z_{l-1 / 2}<z_{l+1 / 2}-\sqrt{\varepsilon}
$$

where $\delta$ is a small positive constant (independent of $\varepsilon$ ). By (10), (91), and Corollary 2.4 ,

$$
\left\{\begin{array}{c}
E_{i}-E_{i+1}=(-1)^{i-l} \mathrm{H}\left(\dot{u}_{l+1 / 2}\right) J\left(z_{i+1 / 2}\right)+O(\varepsilon), \quad i=l, \cdots, k-1 \\
\left(z_{i+1 / 2}-z_{i-1 / 2}\right)+O\left(\left(z_{i-1 / 2}-z_{i+1 / 2}\right)^{2}\right)=\frac{\varepsilon}{\alpha_{i}}\left|\ln f_{i}^{2}\right|+O(\varepsilon) \quad \forall i \\
\left|f_{l}\right|+f_{k} \mid=O\left(e^{-1 / \sqrt{\varepsilon}}\right) .
\end{array}\right.
$$

We now solve, asymptotically for small positive $\varepsilon$, this "algebraic" system for $E_{i}$ and $z_{i+1 / 2}$. For definiteness, we assume that $\dot{u}\left(z_{l+1 / 2}\right)>0$.

Note that $\delta$ small implies $J=o(1)$ in $\left[z^{*}-2 \delta, z^{*}+2 \delta\right]$, so that $E_{i}=o(1)$ and $\tau_{i}-z_{i \pm 1 / 2} \gg \varepsilon$ for all $i=l, \cdots, k$.

3.1. Interior single layer. Suppose $k-l=1$. Then $\left|f_{l}\right|+\left|f_{l+1}\right|=O\left(e^{-1 / \sqrt{\varepsilon}}\right)$ so that $J\left(z_{l+1 / 2}\right)=O(\varepsilon)$. Since $\dot{J}\left(z^{*}\right) \neq 0$, we must have $z_{l+1 / 2}=z^{*}+O(\varepsilon)$. In this case we must have $z^{*} \in(a, b)$, since otherwise we would have $k-l \geq 2$.

3.2. Interior multiple layers. Suppose $0 \leq l, k \leq n$ and $k-l>1$. Then $z^{*} \in(a, b)$. As $z_{l+3 / 2}-z_{l+1 / 2} \gg \varepsilon$,

$$
E_{l+2}-E_{l}=J\left(z_{l+3 / 2}\right)-J\left(z_{l+1 / 2}\right)+O(\varepsilon)=\left\{\dot{J}\left(z^{*}\right)+o(1)\right\}\left(z_{l+3 / 2}-z_{l+1 / 2}\right) .
$$

From $E_{l}=O\left(e^{-1 / \sqrt{\varepsilon}}\right)$, we conclude that $\dot{J}\left(z^{*}\right)>0$. As $J\left(z^{*}\right)=0$, we see that $\phi_{0}\left(z^{*}-\right)=0$ and $\phi_{0}\left(z^{*}+\right)=1$. Since $\dot{u}_{l+1 / 2}>0$, we conclude that $u\left(\tau_{l}\right)=$ $\phi_{0}\left(z^{*}-\right)+O\left(e^{-1 / \sqrt{\varepsilon}}\right)$. In a similar manner, we have $u\left(\tau_{k}\right)=\phi_{0}\left(z^{*}+\right)+O\left(e^{-1 / \sqrt{\varepsilon}}\right)$. Thus, $k-l$ is odd.

A mathematical induction gives, for all positive integers $m \leq(k-l-1) / 2$,

$$
\begin{aligned}
E_{l+2 m} & =\left\{\dot{J}\left(z^{*}\right)+o(1)\right\} \sum_{s=1}^{m}\left(z_{l+2 s-1 / 2}-z_{l+2 s-3 / 2}\right), \\
E_{k-2 m} & =\left\{\dot{J}\left(z^{*}\right)+o(1)\right\} \sum_{s=1}^{m}\left(z_{k-2 s+3 / 2}-z_{k-2 s+1 / 2}\right) .
\end{aligned}
$$

Note that $f_{i}^{2}=2 \alpha_{i}^{2} E_{i}\left(1+O\left(\sqrt{E_{i}}\right)\right)$. Hence,

$$
\begin{aligned}
E_{i}=o(1) & \Longrightarrow z_{i+1 / 2}-z_{i-1 / 2} \gg \varepsilon \Longrightarrow E_{i}>\varepsilon \\
& \Longrightarrow z_{i+1 / 2}-z_{i-1 / 2}=\frac{\varepsilon}{\alpha_{i}}\left|\ln f_{i}^{2}\right|+O(\varepsilon) \leq O(1) \varepsilon|\ln \varepsilon| \\
& \Longrightarrow E_{i} \leq O(1) \varepsilon|\ln \varepsilon| \Longrightarrow \varepsilon \leq E_{i} \leq O(1) \varepsilon|\ln \varepsilon| \\
& \Longrightarrow \frac{\left|\ln E_{i}\right|}{|\ln \varepsilon|}=1+\frac{O(\ln |\ln \varepsilon|)}{|\ln \varepsilon|}=1+o(1) \\
& \Longrightarrow\left|\ln f_{i}^{2}\right|=[1+o(1)]|\ln \varepsilon|
\end{aligned}
$$


for all $i=l+1, \cdots, k-1$. Therefore, $z_{i+1 / 2}-z_{i-1 / 2}=\varepsilon|\ln \varepsilon|\left\{\frac{1}{\alpha_{i}}+o(1)\right\}$, and

$$
\left\{\begin{array}{l}
f_{l+2 m}^{2}=2 m \varepsilon|\ln \varepsilon|\left\{\frac{\alpha_{l+2}^{2}}{\alpha_{l+1}} \dot{J}\left(z^{*}\right)+o(1)\right\}, \\
f_{k-2 m}^{2}=2 m \varepsilon|\ln \varepsilon|\left\{\frac{\alpha_{k-2}^{2}}{\alpha_{k-1}} \dot{J}\left(z^{*}\right)+o(1)\right\},
\end{array} \quad m=1, \cdots,(k-l-1) / 2 .\right.
$$

Finally, using $E_{l+1}=\left[\dot{J}\left(z^{*}\right)+o(1)\right]\left(z^{*}-z_{l+1 / 2}\right)+O(\varepsilon)$ we derive that

$$
z_{i-1 / 2}-z^{*}=\varepsilon|\ln \varepsilon|\left\{o(1)+\left[\frac{i-k}{2}\right] \frac{1}{\alpha_{k-1}}+\left[\frac{i-l}{2}\right] \frac{1}{\alpha_{l+1}}\right\}, \quad i=l+1, \cdots, k,
$$

where $[x]$ represents the largest integer no bigger than $x$. In particular, when $f^{u}\left(0, z^{*}\right)=f^{u}\left(1, z^{*}\right)=\alpha^{2}$, we have

$$
\begin{aligned}
& z_{j}=z^{*}+\frac{\varepsilon|\ln \varepsilon|}{\alpha}\left\{j-\frac{k+l}{2}+o(1)\right\} \text { for } j=l+\frac{1}{2}, l+\frac{3}{2}, \ldots, k-\frac{1}{2}, \\
& \tau_{j}=z^{*}+\frac{\varepsilon|\ln \varepsilon|}{\alpha}\left\{j-\frac{k+l}{2}+o(1)\right\} \text { for } j=l+1, \ldots, k-1 .
\end{aligned}
$$

3.3. Boundary layers. Finally, we consider the case $k=-l$. Then necessarily, we must have $z^{*}=a$. Restricting to the interval $[a, b]$, we have the "boundary conditions"

$$
E_{k}=O\left(e^{-1 / \sqrt{\varepsilon}}\right), \quad z_{1 / 2}=a+\left\{\frac{1}{2 \alpha_{0}}+o(1)\right\} \varepsilon\left|\ln f_{0}^{2}\right| .
$$

Following a similar argument as for interior layers, we conclude that $\dot{J}(a) \dot{u}_{k-1 / 2}>0$ or $u\left(\tau_{k}\right)=\phi_{0}(a+)+O\left(e^{-1 / \sqrt{\varepsilon}}\right)$. Also for $i=1, \cdots, k$ and $m=0, \cdots,\left[\frac{k-1}{2}\right]$,

$$
\begin{aligned}
& z_{i-1 / 2}-a=\left\{o(1)+\left(\left[\frac{i+1}{2}\right]-\frac{1}{2}\right) \frac{1}{\alpha_{0}}+\left[\frac{i}{2}\right] \frac{1}{\alpha_{1}}\right\} \varepsilon|\ln \varepsilon|, \\
& f_{k-2 m}^{2}=2 \alpha_{k-2}^{2}|\dot{J}(a)|\left\{\frac{m}{\alpha_{k-1}}+o(1)\right\} \varepsilon|\ln \varepsilon|, \\
& f_{k-2 m-1}^{2}=2 \alpha_{k-1}^{2}|\dot{J}(a)|\left\{\frac{k}{2 \alpha_{k-1}}+\frac{k-2 m-1}{2 \alpha_{k-2}}+o(1)\right\} \varepsilon|\ln \varepsilon| .
\end{aligned}
$$

In summary, we have the following:

Theorem 2. Let $N$ be a given positive integer. There exist positive constants $\delta^{*}$ and $\varepsilon^{*}$ such that for each $z^{*} \in Z_{J}$, the following hold:

Let $\varepsilon \in\left(0, \varepsilon^{*}\right]$ and $u \in \mathcal{S}_{N, \varepsilon}$ be arbitrary. Suppose that $\left(\tau_{l}, \tau_{k}\right)$ is an interval with a cluster for $u$ such that $\left[z_{l+1 / 2}, z_{k-1 / 2}\right] \cap\left[z^{*}-\delta^{*}, z^{*}+\delta^{*}\right]$ is non-empty. Then the following hold:

(i) If $z^{*} \in(a, b)$ and $k-l=1$, then $z_{l+1 / 2}=z^{*}+O(\varepsilon)$.

(ii) If $z^{*} \in(a, b)$ and $k-l>1$, then $k-l$ must be odd, $\dot{J}\left(z^{*}\right) \dot{u}_{l+1 / 2}>0$, and (11) -(12) holds.

(iii) If $z^{*}=a$, then $l=-k, \dot{J}\left(a^{*}\right) \dot{u}_{k-1 / 2}>0$, and (13) holds.

(iv) If $z^{*}=b$, then $k=2 n-l, \dot{J}\left(b^{*}\right) \dot{u}_{l+1 / 2}>0$, and relations analogous to (13) hold.

Remark 3.1. The estimates (11) or (13) imply that the minima and maxima of $u$ in $\left[\tau_{l}, \tau_{k}\right] \cap[a, b]$ are monotonic, which is a key observation used in $\underline{\mathrm{AH}}$.

\section{SPIKES}

Theorem 2 implies that if $\left(\tau_{l}, \tau_{k}\right)$ is an interval with a cluster, then either $\left[z_{l+1 / 2}, z_{k-1 / 2}\right] \subset[z-O(\varepsilon|\ln \varepsilon|), z+O(\varepsilon|\ln \varepsilon|)]$ for some $z \in Z_{J}$ or $\left[z_{l+1 / 2}, z_{k-1 / 2}\right] \cap$ $\left(\bigcup_{z \in Z_{J}}\left[z-\delta^{*}, z+\delta^{*}\right]\right)=\emptyset$. In this section, we consider the latter case, i.e., an interval $\left(\tau_{l}, \tau_{k}\right)$ of a cluster such that

$$
\left[z_{l+1 / 2}, z_{k-1 / 2}\right] \cap\left(\bigcup_{z \in Z_{J}}\left[z-\delta^{*}, z+\delta^{*}\right]\right)=\emptyset .
$$


Note that there exists a positive constant $\eta$ which is independent of $\varepsilon \in\left(0, \varepsilon^{*}\right]$ such that either $J>\eta$ in $\left[z_{l+1 / 2}, z_{k-1 / 2}\right]$ or $J<-\eta$ in $\left[z_{l+1 / 2}, z_{k-1 / 2}\right]$. It follows from Lemma 2.1 that $\varepsilon^{2} \dot{u}^{2}(x)=F(u(x), x)+o(1)$ for all $x \in\left[\tau_{l}, \tau_{k}\right]$. Hence, $k-l$ must be even, $u_{l+2 m-1}=\phi^{*}{ }_{l+2 m-1}+o(1)$ and $z_{l+2 m-1 / 2}-z_{l+2 m-3 / 2}=O(\varepsilon)$ for all $m=1, \cdots,(k-l) / 2$, and for all $m=0, \cdots,(k-l) / 2, u_{l+2 m}=o(1)$ if $J<0$ or $u_{l+2 m}=1-o(1)$ if $J>0$.

For definiteness, we shall assume that $J<-\eta$ in $\left[z_{l+1 / 2}, z_{k-1 / 2}\right]$ and define $F(v, x)=\int_{0}^{v} f(s, x) d s$ for all $x \in\left[\tau_{l}, \tau_{k}\right]$.

Lemma 4.1. For each integer $i=l+1, l+3, \cdots, k-1$ (i.e., $\tau_{i}$ is the peak of a spike),

$$
\begin{gathered}
2 \alpha_{i \pm 1}\left|\tau_{i}-\tau_{i \pm 1}\right|+O\left(\left|\tau_{i}-\tau_{i \pm 1}\right|^{2}\right)=\varepsilon\left|\ln F_{i \pm 1}\right|+O(\varepsilon), \\
F_{i}-F_{i \pm 1}= \pm \varepsilon \mathrm{H}\left(J\left(\tau_{i}\right)\right) S\left(\tau_{i}\right)+O\left(\varepsilon^{2}+\varepsilon F_{i \pm 1}\left|\ln F_{i \pm 1}\right|\right) .
\end{gathered}
$$

Proof. Since $J<-\eta$ in $\left[z_{l+1 / 2}, z_{k-1 / 2}\right]$ and $u_{i}=\phi_{i}^{*}+o(1), f_{i}<-\tilde{\eta}$ and $\tau_{i}$ is a local maximum. By (9), $z_{i+1 / 2}-z_{i-1 / 2}=O(\varepsilon)$. As $\ln f_{i \pm 1}^{2}=\ln F_{i \pm 1}+O(1)$, (15) follows from (91). It remains to prove (16).

Integrating $\frac{d}{d t}\left(F(u(t), t)-\frac{1}{2} \varepsilon^{2} \dot{u}^{2}(t)\right)=F_{x}(u(t), t)$ over $\left(\tau_{i-1}, \tau_{i}\right)$, we obtain

$$
F_{i}-F_{i-1}=\left(\int_{\tau_{i-1}}^{z_{i-1 / 2}}+\int_{z_{i-1 / 2}}^{\tau_{i}}\right) F_{x}(u(t), t) d t .
$$

In the interval $t \in\left[\tau_{i-1}, z_{i-1 / 2}\right]$, we use $F_{x}(u, t)=F_{x}\left(u, \tau_{i}\right)+O\left(u^{2}\right)\left(\tau_{i}-t\right)=$ $O\left(u^{2}\right), d t=\varepsilon \frac{1+O(\varepsilon)}{\sqrt{2 G\left(u, u_{i-1}, t\right)}} d u(t), G\left(u, u_{i-1}, t\right)=G\left(u, u_{i-1}, \tau_{i}\right)\left[1+O\left(\tau_{i}-t\right)\right], \tau_{i}-t=$ $O(\varepsilon)+z_{i-1 / 2}-t$, and $z_{i-1 / 2}-t=O(\varepsilon) \int_{u}^{\phi} \frac{1}{\sqrt{s^{2}-u_{i-1}^{2}}} d s=O(\varepsilon) \ln u$ to conclude that

$$
\begin{aligned}
& \int_{\tau_{i-1}}^{z_{i-1 / 2}} F_{x}(u(t), t) d t=\varepsilon \int_{u_{i-1}}^{\phi_{i-1 / 2}}\left\{\frac{F_{x}\left(s, \tau_{i}\right)}{\left(2 G\left(s, u_{i-1}, \tau_{i}\right)\right)^{1 / 2}}+\frac{O(\varepsilon) s^{2} \ln s}{\left(s^{2}-u_{i-1}^{2}\right)^{1 / 2}}\right\} d s \\
= & \varepsilon\left\{\int_{0}^{\phi_{i-1 / 2}}-\int_{0}^{u_{i-1}}\right\} \frac{F_{x}\left(s, \tau_{i}\right)}{\sqrt{2 F\left(s, \tau_{i}\right)}} d s \\
& +\varepsilon \int_{u_{i-1}}^{\phi_{i-1 / 2}} F_{x}\left(s, \tau_{i}\right)\left\{\frac{1}{\sqrt{2 G\left(s, u_{i-1}, \tau_{i}\right)}}-\frac{1}{\sqrt{2 F\left(s, \tau_{i}\right)}}\right\} d s+O\left(\varepsilon^{2}\right) \\
= & \varepsilon \int_{0}^{\phi_{i-1 / 2}} \frac{F_{x}\left(s, \tau_{i}\right)}{\sqrt{2 F\left(s, \tau_{i}\right)}} d s+O(\varepsilon) u_{i-1}^{2} \ln u_{i-1}+O\left(\varepsilon^{2}\right)
\end{aligned}
$$

since $\left|\frac{F_{x}(s, x)}{\sqrt{2 F(s, x)}}\right|=O(s),\left|F_{x}\right|\left|\frac{1}{\sqrt{G}}-\frac{1}{\sqrt{F}}\right| \leq \frac{\left|F_{x} \| F-G\right|}{F \sqrt{G}}=O(1) \frac{u_{i-1}^{2}}{\sqrt{s^{2}-u_{i-1}^{2}}}$. It then follows that

$$
\int_{\tau_{i-1}}^{z_{i-1 / 2}} F_{x}(u(t), t) d t=\varepsilon \int_{0}^{\phi_{i-1 / 2}} \frac{F_{x}\left(s, \tau_{i}\right)}{\sqrt{2 F\left(s, \tau_{i}\right)}} d s+O\left(\varepsilon^{2}\right)+O\left(\varepsilon F_{i-1}\left|\ln F_{i-1}\right|\right) .
$$

To estimate $\int_{z_{i-1 / 2}}^{\tau_{i}} F_{x} d t$, we first note that the integral is $O(\varepsilon)$ since $\left|\tau_{i}-z_{i-1 / 2}\right|=$ $O(\varepsilon)$. This implies that $F_{i}=F_{i-1}+O(\varepsilon)$. An induction then gives us $F_{j}=O(\varepsilon)$ for all $j=l, \cdots, k$. Consequently $u_{i}=\phi^{*}\left(\tau_{i}\right)+O(\varepsilon)$. We next show that

$$
\int_{z_{i-1 / 2}}^{\tau_{i}} F_{x}(u(t), t) d t=\varepsilon \int_{\phi_{i-1 / 2}}^{\phi_{i}^{*}} \frac{F_{x}\left(s, \tau_{i}\right)}{\sqrt{2 F\left(s, \tau_{i}\right)}} d s+O\left(\varepsilon^{2}\right) .
$$


We first note that, for $t \in\left[z_{i-1 / 2}, \tau_{i}\right], G\left(u, u_{i}, t\right) \propto\left(u_{i}-u\right), F_{x}(u, t)=F_{x}\left(u, \tau_{i}\right)+$ $O(\varepsilon)$ and $G\left(u, u_{i}, t\right)=G\left(u, u_{i}, \tau_{i}\right)[1+O(\varepsilon)]$. It then follows that

$$
\int_{z_{i-1 / 2}}^{\tau_{i}} F_{x}(u(t), t) d t=\varepsilon \int_{u_{i-1 / 2}}^{u_{i}} \frac{F_{x}\left(s, \tau_{i}\right)}{\sqrt{2 G\left(s, u_{i}, \tau_{i}\right)}} d s+O\left(\varepsilon^{2}\right) .
$$

Now letting $\hat{s}=s+\phi_{i}^{*}-u_{i}$ yields

$$
\int_{z_{i-1 / 2}}^{\tau_{i}} F_{x}(u(t), t) d t=\varepsilon \int_{u_{i-1 / 2}+\phi_{i}^{*}-u_{i}}^{\phi_{i}^{*}} \frac{F_{x}\left(\hat{s}-\phi_{i}^{*}+u_{i}, \tau_{i}\right)}{\sqrt{2 G\left(\hat{s}-\phi_{i}^{*}+u_{i}, u_{i}, \tau_{i}\right)}} d \hat{s}+O\left(\varepsilon^{2}\right) .
$$

Note that $F_{x}\left(\hat{s}-\phi_{i}^{*}+u_{i}, \tau_{i}\right)=F_{x}\left(\hat{s}, \tau_{i}\right)+O(\varepsilon), G\left(\hat{s}-\phi_{i}^{*}+u_{i}, u_{i}, \tau_{i}\right)=G\left(\hat{s}, \phi_{i}^{*}, \tau_{i}\right)=$ $[1+O(\varepsilon)] F\left(\hat{s}, \tau_{i}\right)$ and $u_{i-1 / 2}=\phi_{i-1 / 2}$. We have

$$
\begin{aligned}
\int_{z_{i-1 / 2}}^{\tau_{i}} F_{x}(u(t), t) d t=\varepsilon & \int_{\phi_{i-1 / 2}}^{\phi_{i}^{*}} \frac{F_{x}\left(\hat{s}, \tau_{i}\right)}{\sqrt{2 F\left(\hat{s}, \tau_{i}\right)}} d \hat{s} \\
& +\varepsilon \int_{\phi_{i-1 / 2}+\phi_{i}^{*}-u_{i}}^{\phi_{i-1 / 2}} \frac{F_{x}\left(\hat{s}, \tau_{i}\right)}{\sqrt{2 F\left(\hat{s}, \tau_{i}\right)}} d \hat{s}+O\left(\varepsilon^{2}\right),
\end{aligned}
$$

and then (17) since

$$
\int_{\phi_{i-1 / 2}+\phi_{i}^{*}-u_{i}}^{\phi_{i-1 / 2}} \frac{F_{x}\left(\hat{s}, \tau_{i}\right)}{\sqrt{2 F\left(\hat{s}, \tau_{i}\right)}} d \hat{s}=O\left(\left|\phi_{i}^{*}-u_{i}\right|\right)=O(\varepsilon) .
$$

Therefore, we have $F_{i}-F_{i-1}=\varepsilon \int_{0}^{\phi^{*}{ }_{i}} \frac{F_{x}\left(s, \tau_{i}\right)}{\sqrt{2 F\left(s, \tau_{i}\right)}} d s+O\left(\varepsilon^{2}+\varepsilon F_{i-1}\left|\ln F_{i-1}\right|\right)=$ $-\varepsilon \mathrm{H}\left(J\left(\tau_{i}\right)\right) S\left(\tau_{i}\right)+O\left(\varepsilon^{2}+\varepsilon F_{i-1}\left|\ln F_{i-1}\right|\right)$. The cases for $F_{i}-F_{i+1}$ and for $J>\eta$ can be similarly derived. This completes the proof.

We now study a generic cluster away from $Z_{J}$.

4.1. Interior single spike. Suppose $0 \leq l$ and $k=l+2 \leq n$. Then $F_{l}=F_{l+2}=$ $O\left(e^{-c / \sqrt{\varepsilon}}\right)$, so that by (16),

$$
2 \varepsilon \mathrm{H}\left(J\left(\tau_{l+1}\right)\right) S\left(\tau_{l+1}\right)=F_{l}-F_{l+2}+O\left(\varepsilon^{2}+\varepsilon F_{l}\left|\ln F_{l}\right|+\varepsilon F_{l+2}\left|\ln F_{l+2}\right|\right)=O\left(\varepsilon^{2}\right) .
$$

Therefore, we must have

$$
S\left(\tau_{l+1}\right)=O(\varepsilon), \quad F_{l+1}=O\left(\varepsilon^{2}\right) .
$$

This implies that there exists $z^{*} \in[a, b]$ such that $S\left(z^{*}\right)=0$ and $\tau_{l+1}=z^{*}+O(\varepsilon)$. In addition, since $u$ is even about $a$ and $b$, we must have $z^{*} \in(a, b)$.

4.2. Interior multiple spikes. Next we consider the case $0 \leq l$ and $l+2<k \leq n$. One can proceed step by step to derive the following:

1) $F_{i}=O(\varepsilon)$ for all $i=l, \cdots, k$. Consequently, $\tau_{i+1}-\tau_{i} \gg \varepsilon$ for all $i=$ $l, \cdots, k-1$.

2) There must hold $\left|S\left(\tau_{l+2 m-1}\right)\right|<2 \sqrt{\varepsilon}$ for all $m=1, \cdots,(k-l) / 2$. Indeed, if $S\left(\tau_{l+2 m-1}\right)>2 \sqrt{\varepsilon}$, then $S(x)>\sqrt{\varepsilon}$ in $\left[\tau_{l+2 m-1}-\varepsilon^{3 / 4}, \tau_{l+2 m-1}+\varepsilon^{3 / 4}\right]$. This implies, by an induction, that $F\left(\tau_{l+2 m+i}\right)>\varepsilon^{3 / 2}$ and $\tau_{l+2 m+i+1}-\tau_{l+2 m+i} \leq C \varepsilon|\ln \varepsilon|$ for $i=1,2, \cdots$ all the way up to $\tau_{l+2 m+i+1}-\tau_{l+2 m}>\varepsilon^{3 / 4}$. Hence, $u$ will have at least $\varepsilon^{3 / 4} /(C \varepsilon|\ln \varepsilon|)$ oscillations, contradicting our assumption that $u \in \mathcal{S}_{N, \varepsilon}$. Similarly, we can exclude the case $S\left(\tau_{l+2 m-1}\right)<-2 \sqrt{\varepsilon}$. Hence, we must have $\left|S\left(\tau_{l+2 m-1}\right)\right|<2 \sqrt{\varepsilon}$ for all $m=1, \cdots,(k-l) / 2$. Hence, there exists $z^{*} \in[a, b]$ such that $S\left(z^{*}\right)=0$ and $\tau_{i}=z^{*}+O(\sqrt{\varepsilon})$ for all $i=1+1, \cdots, k-1$. As $0 \leq l$ and $k \leq n$, we must have $z^{*} \in(a, b)$. 
For definiteness, we assume that $J\left(z^{*}\right)<-\eta$, so that $f_{l+2 m-1}<0$ for $m=$ $1, \cdots,(k-l) / 2$.

3) From Step 2 and (16), we see that $F_{i}=O\left(\varepsilon^{3 / 2}\right)$ for all $i=l, \cdots, k$. Consequently,

$$
\left\{\begin{array}{l}
F_{l+2 m}-F_{l+2 m-1}=\varepsilon S\left(\tau_{l+2 m-1}\right)+O\left(\varepsilon^{2}\right), \\
F_{l+2 m-1}-F_{l+2 m-2}=\varepsilon S\left(\tau_{l+2 m-1}\right)+O\left(\varepsilon^{2}\right), \quad \forall m=1, \cdots,(k-l) / 2 . \\
F_{l+2 m}-F_{l+2 m-2}=2 \varepsilon S\left(\tau_{l+2 m-1}\right)+O\left(\varepsilon^{2}\right)
\end{array}\right.
$$

From this, we conclude that $\dot{S}\left(z^{*}\right)<0$, i.e., $J\left(z^{*}\right) \dot{S}\left(z^{*}\right)>0$, since $0<F_{l+2}+$ $F_{k-2}=2 \varepsilon S\left(\tau_{l+1}\right)-2 \varepsilon S\left(\tau_{k-1}\right)+O\left(\varepsilon^{2}\right)=\left\{o(1)-2 \dot{S}\left(z^{*}\right)\right\} \varepsilon\left(\tau_{k-1}-\tau_{l+1}\right)$ (recalling $\left.\tau_{i+1}-\tau_{i} \gg \varepsilon\right)$.

4) For all $m=1, \cdots,(k-l) / 2-1$,

$$
\begin{aligned}
0<F_{l+2 m+2}+F_{l+2 m-2} & =2 F_{l+2 m}+2 \varepsilon\left\{S\left(\tau_{l+2 m+1}\right)-S\left(\tau_{l+2 m-1}\right\}\right)+O\left(\varepsilon^{2}\right) \\
& =2 F_{l+2 m}+2 \varepsilon\left[\dot{S}\left(z^{*}\right)+o(1)\right]\left(\tau_{l+2 m+1}-\tau_{l+2 m-1}\right) .
\end{aligned}
$$

It then follows that $F_{l+2 m} \gg \varepsilon^{2}$ for all $m=1, \cdots,(k-l) / 2-1$, since $\dot{S}\left(z^{*}\right)<0$.

Once we know $\varepsilon^{2}<F_{l+2 m}$, we can conclude from (15) that $\left|\tau_{l+2 m \pm 1}-\tau_{l+2 m}\right| \leq$ $C \varepsilon|\ln \varepsilon|$ and $F_{l+2 m+2}+F_{l+2 m-2}-2 F_{l+2 m}=O\left(\varepsilon^{2}|\ln \varepsilon|\right)$. The "boundary condition" $F_{l}, F_{k}=O\left(e^{-1 / \sqrt{\varepsilon}}\right)$ then gives us $F_{l+2 m}=O\left(\varepsilon^{2} \ln \varepsilon\right)$ for all $m=0, \cdots,(k-l) / 2$.

5) From $\varepsilon^{2}<F_{l+2 m}<O(1) \varepsilon^{2}|\ln \varepsilon|$, i.e., $\left|\ln F_{l+2 m}\right|=\{1+o(1)\} 2|\ln \varepsilon|$ for all $m=1, \cdots, k / 2-1$, we derive from (15) that $\tau_{i+1}-\tau_{i}=\left\{\frac{1}{\alpha^{*}}+o(1)\right\} \varepsilon|\ln \varepsilon|$ for all $i=1+1, \cdots, k-2$, where $\alpha^{*}=\sqrt{f_{u}\left(0, z^{*}\right)}\left(=\sqrt{f_{u}\left(\phi_{0}\left(z^{*}\right), z^{*}\right)}\right)$.

6) From step 5), we see that

$$
\begin{aligned}
F_{l+2 m+2}+F_{l+2 m-2}-2 F_{l+2 m} & =2 \varepsilon\left\{S\left(\tau_{l+2 m+1}\right)-S\left(\tau_{l+2 m-1}\right)\right\}+O\left(\varepsilon^{2}\right) \\
& =\left\{\frac{4}{\alpha^{*}} \dot{S}\left(z^{*}\right)+o(1)\right\} \varepsilon^{2}|\ln \varepsilon|
\end{aligned}
$$

for $m=1, \cdots,(k-l-2) / 2$. Using $F_{l}, F_{k}=O\left(e^{-c / \sqrt{\varepsilon}}\right)$ we then conclude that (18)

$$
F_{i}= \begin{cases}\left\{\frac{(k-i)(i-l)}{2 \alpha^{*}}\left|\dot{S}\left(z^{*}\right)\right|+o(1)\right\} \varepsilon^{2}|\ln \varepsilon|, & i=l, l+2, \cdots, k, \\ \left\{\frac{(k-i)(i-l)-1}{2 \alpha^{*}}\left|\dot{S}\left(z^{*}\right)\right|+o(1)\right\} \varepsilon^{2}|\ln \varepsilon|, & i=l+1, l+3, \cdots, k-1 .\end{cases}
$$

Consequently, using $F_{l+2}=2 \varepsilon S\left(\tau_{l+1}\right)+O\left(\varepsilon^{2}\right)=2 \varepsilon\left\{o(1)+S\left(z^{*}\right)\right\}\left(\tau_{l+1}-z^{*}\right)+$ $O\left(\varepsilon^{2}\right)$ we then conclude that

$$
\tau_{i}=z^{*}+\left\{\frac{2 i-(k+l)}{2 \alpha^{*}}+o(1)\right\} \varepsilon|\ln \varepsilon|, \quad i=l+1, \cdots, k-1 .
$$

4.3. Boundary spikes. Finally we consider the case $l=-k$.

Suppose $S(a)=0$. Then $S(\cdot)$ is $C^{1}$ in a neighborhood of $a$ since $S$ is an odd function. Hence, the same conclusion as for the interior spikes holds.

Suppose $S(a) \neq 0$. From Lemma 4.1 and $F_{k}=O\left(e^{-1 / \sqrt{\varepsilon}}\right)$ we then derive that

$$
\left\{\begin{array}{l}
F_{i}=\varepsilon(k-i) \mathrm{H}(J(a)) S(a)+O\left(\varepsilon^{2}|\ln \varepsilon|\right), \quad \forall i=0, \cdots, k-1, \\
\tau_{i}=a+\left\{\frac{i}{2 \alpha^{*}}+o(1)\right\} \varepsilon|\ln \varepsilon|
\end{array}\right.
$$

where $\alpha^{*}=\sqrt{\left.f_{u}\left(\phi_{0}(a), a\right), a\right)}$. One notes that if $k \geq 2$, we must have $J(a) S(a)>0$. (The analogous relation at $b$ is $F_{i}=\varepsilon(l-i) \mathrm{H}(J(b)) S(b)+O\left(\varepsilon^{2}|\ln \varepsilon|\right)$ for $i=l, \cdots, n$ and $J(b) S(b)<0$ when $l \leq n-2$.) 
In summary, we have the following theorem.

Theorem 3. Assume (2), (41), and (5). Let $N$ be fixed, and let $\delta^{*}$ and $\varepsilon^{*}$ be as in Theorem 2. Then there exist $\tilde{\varepsilon}^{*} \in\left(0, \varepsilon^{*}\right]$ such that the following is true.

Let $u \in \mathcal{S}_{N, \varepsilon}$ with $\varepsilon \in\left(0, \tilde{\varepsilon}^{*}\right]$, and let $\left(\tau_{l}, \tau_{k}\right)$ be an interval with a cluster such that $\left[z_{l+1 / 2}, z_{k-1 / 2}\right] \cap\left[z-\delta^{*}, z+\delta^{*}\right]$ is empty for all $z \in Z_{J}$. Then $k-l$ is even and there exists $z^{*} \in Z_{S}$ such that $u\left(\tau_{l}\right)=\phi_{0}\left(z^{*}\right)+O\left(e^{-1 / \sqrt{\varepsilon}}\right)$ and $u\left(\tau_{k}\right)=\phi_{0}\left(z^{*}\right)+$ $O\left(e^{-1 / \sqrt{\varepsilon}}\right)$. In addition,

(i) if $k-l=2$, then $\tau_{l+1}=z^{*}+O(\varepsilon), u_{l+1}=\phi^{*}\left(\tau_{l+1}\right)+O\left(\varepsilon^{2}\right)$, and $F_{l+1}=O\left(\varepsilon^{2}\right)$;

(ii) if $z^{*} \in(a, b)$ and $k-l>2$, then $J\left(z^{*}\right) \dot{S}\left(z^{*}\right)>0=S\left(z^{*}\right)$, and (18) and (19)) hold;

(iii) if $z^{*}=a$ and $k=-l>1$, then either $S(a)=0<J(a) \dot{S}(a)$ and (18) and (19) hold or $J(a) S(a+)>0$ and (20) holds;

(vii) if $z^{*}=b$ and $k=2 n-l>n+1$, then either $J(b) \dot{S}(b)>0=S(b)$ or $J(b) S(b-)<0$ and analogous to (18), and (19) or (20) holds.

Clearly, Theorem 1 follows from Theorems 2 and 3 .

\section{Part II: Existence}

Existence of solutions with the properties above, specifically, solutions in $\mathcal{S}_{N, \varepsilon}$ with arbitrarily prescribed numbers of layers and spikes at the places allowed by Theorem 1, will be proved using a shooting technique and induction. We will start with an interval $[0, L]$ and then observe that the technique can be extended to a function $f(u, x)$ with appropriate properties defined on $(-\infty, \infty)$. This is possible because all of the estimates used in the proof of Theorem 1 are local.

For convenience, we assume that, for some positive constant $M$,

$$
\begin{gathered}
\|f\|_{C^{2}([0,1] \times \mathbb{R})}+\|\phi\|_{C^{2}(\mathbb{R})} \leq M, \\
f_{u}(0, x) \geq \frac{1}{M}, \quad f_{u}(1, x)>\frac{1}{M}, \quad 1-\frac{1}{M} \geq \phi(x) \geq \frac{1}{M} \quad \forall x \in \mathbb{R}, \\
J^{2}(x)+\dot{J}^{2}(x) \geq \frac{1}{M}, \quad S^{2}(x)+\dot{S}^{2}(x)>\frac{1}{M} \quad \forall x \in \mathbb{R} .
\end{gathered}
$$

We let $Z=\{\xi>0 \mid J(\xi)=0$ or $S(\xi)=0\}=\left\{\xi_{i}\right\}_{i=1}^{\infty}$, where $\xi_{i}<\xi_{i+1}$ for all $i$. Since $J(\xi)=0<|\dot{J}(\xi)|$ implies $|S(\xi)|=\infty$, the roots of $J=0$ and $S=0$ are distinct. We choose $\delta>0$ such that $\xi_{i+1}-\xi_{i}>6 \delta$ for all $i \geq 1$. Note that $\delta$ depends only on $M$.

For $L \in(0, \infty) \cup\{\infty\}$, we consider

$$
\varepsilon^{2} \ddot{u}=f(u, x) \quad \text { for } \quad x \in(0, L), \quad \dot{u}(0)=0=\dot{u}(L) .
$$

In the sequel, an oscillation refers to a critical point of $\dot{u}$ (roots to $u=\phi$ ). There are two types of oscillations: layers and spikes. A spike contains two oscillations. If boundary oscillations at $\xi_{0}:=0$ are required, we shall assume that $\xi_{1}>6 \delta$. Similarly, if boundary oscillations at $L$ are required, we assume that either $L \in Z$ or $\operatorname{dist}(L, Z) \geq 6 \delta$. We now state our existence theorem.

Theorem 4. Suppose that $N>0$ is an integer and $\delta>0$ is a small constant. Then there is an $\varepsilon_{0}=\varepsilon_{0}(N, M, \delta)>0$ such that if $0<\varepsilon<\varepsilon_{0}, k$ is either a positive integer or $k=\infty,\left\{\sigma_{i}\right\}_{i=0}^{k+1}$ is an arbitrary sequence of the integers from $\{0, \cdots, N\}$, and $L \in\left(\xi_{k}+2 \delta, \infty\right) \cup\{\infty\}$ is an arbitrary constant, then there is a solution of (21) with a cluster of exactly $\sigma_{i}$ oscillations in $\left(\xi_{i}-\sqrt{\varepsilon}, \xi_{i}+\sqrt{\varepsilon}\right)$ for 
$i=1, \cdots, k$, provided that this combination of oscillations is allowed by Theorem 1. Further if $\xi_{1} \geq 6 \delta$, there will be $\sigma_{0}$ boundary oscillations at 0 and, if $L<\infty$ and either $L \in Z$ or $\operatorname{dist}(L, Z) \geq 6 \delta$, there will be $\sigma_{k+1}$ boundary oscillations at $L$, depending on which of these is allowed by Theorem 1. There can be no other oscillations in $(0, L)$.

Remark 4.1. If $J\left(\xi_{i}\right)=0<\sigma_{i}$, then $\sigma_{i}$ is odd and the corresponding oscillations in $\left(\xi_{i}-\sqrt{\varepsilon}, \xi_{i}+\sqrt{\varepsilon}\right)$ are layers. If $S\left(\xi_{i}\right)=0<\sigma_{i}$, then $\sigma_{i}$ is even and the oscillations are spikes. Similarly, the boundary oscillations can be layers or spikes. A half spike at 0 is a boundary spike with $u(0)$ close to $\phi^{*}(0)$.

Remark 4.2. The theorem extends to solutions on $\mathbb{R}$, where $\{\xi\}$ and $\{\sigma\}$ are replaced by double-sided sequences $\left\{\xi_{i}\right\}_{i=-\infty}^{\infty}$ and $\{\sigma\}_{i=-\infty}^{\infty}$, respectively. The assertion follow by two steps: (i) For each $T>0$, apply Theorem 4 for solutions on the interval $[-T, \infty)$ to obtain a solution $u^{T}$ with the desired number of oscillations on $(-T, \infty)$. (ii) Along a sequence of $T_{j} \rightarrow \infty,\left(u^{T_{j}}(0), \dot{u}^{T_{j}}(0)\right)$ will have a limit. Taking this limit as the initial value, we then obtain the desired solution.

\section{A Shooting ARGument}

5.1. The shooting argument. We use ideas from [A, $\mathrm{AH}, \mathrm{HM}$ by considering the initial value problem

$$
\varepsilon^{2} \ddot{u}=f(u, x), \quad u(0)=\alpha, \quad \dot{u}(0)=0,
$$

where $\alpha$ is the shooting parameter. Since the desired solutions take values in $(0,1)$, $f$ for large $|u|$ can be modified to be uniformly bounded. Hence, (22) admits a unique solution for all $x \in \mathbb{R}$. If necessary, we write the solution as $u(\cdot, \alpha)$.

A critical point of $u$ is a point at which $\dot{u}=0$; it is called non-degenerate if $\ddot{u} \neq 0$ at the point. Similarly, since $\ddot{u}=0 \Leftrightarrow u=\phi$, a critical point of $\dot{u}$ is a point at which $u=\phi$ (i.e., $\ddot{u}=0$ ); it is called non-degenerate if at the point $\dot{u} \neq \dot{\phi}$. Note that if $\hat{\tau}$ is a non-degenerate critical point of $u(\cdot, \hat{\alpha})$, then by the Implicit Function Theorem, $\dot{u}(\tau, \alpha)=0$ has a unique smooth solution $\tau=\tau(\alpha)$ with $\hat{\tau}=\tau(\hat{\alpha})$ for all $\alpha$ close to $\hat{\alpha}$. A similar property holds also for solutions to $u(z, \alpha)-\phi(z)=0$.

We let

$$
\mu=\frac{1}{2} \inf _{x \geq 0} \min \left\{\left|\phi(x)-\phi_{0}(x)\right|,\left|\phi(x)-\phi^{*}(x)\right|\right\} .
$$

Lemma 5.1. Suppose $N>0$. Then there is an $\varepsilon_{0}>0$ such that if $0<\varepsilon<\varepsilon_{0}$ and $u$ is a solution of (22) with $\alpha \in(0, \phi(0)-\mu) \cup(\phi(0)+\mu, 1)$ and with at most $N$ critical points in any subinterval of $[0, X]$ of length less than $\delta$, then all critical points of $u$ and $\dot{u}$ in $[0, X]$ are non-degenerate and interlace. In addition, if $\tau_{m}$ is the last critical point of $u$ in $[0, X]$, then the first critical point $z_{m+1 / 2}$ of $\dot{u}$ in $\left(\tau_{m}, \infty\right)$ exists and is also non-degenerate.

Proof. From the formula

$$
\frac{d}{d x}\left(\varepsilon^{2} \dot{u}^{2}-2 F(u, x)\right)=-2 F_{x}(u, x)
$$

we see that the change in $F(u, x)$ between any two critical points of $u$ in $[0, X]$ tends to zero as $X \rightarrow 0$. This means that we can choose $\delta_{1}>0$ (independent of $\varepsilon$ ) so small that if $X \leq \delta_{1}$, then the conclusion of the lemma follows. If $X>\delta_{1}$, then the result is implied by Lemma 2.1 . 
In the sequel, $\tau_{0}=0$ and the $i$ th critical point of $u(\cdot, \alpha)$ in $(0, \infty)$, if it exists, is denoted by $\tau_{i}(\alpha)$, whereas the $i$ th critical point of $\dot{u}$ is denoted by $z_{i-1 / 2}(\alpha)$.

A crucial result in proving existence is the following extension of Lemma 4.1

Lemma 5.2. Assume that $u$ solves $\varepsilon^{2} \ddot{u}=f(u, x)$ on $\mathbb{R}$ and $z$ and $\tau$ are points such that

$$
\dot{u}(\tau)=0, \quad \dot{u}<0 \quad \text { on }(\tau, z], \quad \tau+\delta<z, \quad u(z)=\phi(z), \quad \operatorname{dist}(z, Z) \geq \delta .
$$

Then the following hold:

(a) If $J(z)>0$ and $S(z)<0$, then there are at least $N$ critical points of $u$ in $[z, z+\sqrt{\varepsilon}]$.

(b) If $J(z)>0$ and $S(z)>0$, then $u$ has exactly one critical point (indeed the only minimum) in $(\tau, \infty)$, and it exceeds 1 after a finite $x>z$.

(c) If $J(z)<0$, then $\dot{u}<0$ in $(\tau, \infty)$ and $u$ becomes negative after a finite $x>z$.

Proof. The condition $\operatorname{dist}(z, Z) \geq \delta$ implies that $J(z)$ and $S(z)$ are bounded away from 0 , uniformly in $\varepsilon$. We set

$$
K=\sqrt{\left\|f_{u}\right\|_{L^{\infty}}}, \quad \nu:=\inf _{x \in \mathbb{R},(1+\phi(x)) / 2<s<1} \sqrt{\frac{f(s, x)}{s-1}}>0 .
$$

Following the proof of (8) in Lemma 2.3 , we obtain

$$
\varepsilon^{2} \dot{u}^{2}(x)=[2+O(\varepsilon)] G(u(x), u(\tau), x)
$$

for all $x \in[\tau, z]$. Since $z-\tau \geq \delta$, a calculation similar to that for (9) then gives $u(\tau)=1-O\left(e^{-\delta \nu / \varepsilon}\right)$. Hence, $\varepsilon^{2} \dot{u}^{2}(z)=2 G(\phi(z), 1, z)+O(\varepsilon)$. Thus, by a continuous dependence argument,

$$
u(z+\varepsilon y)=U(y)+O(\varepsilon) e^{K|y|} \quad \forall y \in \mathbb{R},
$$

where $U$ is the unique solution to

$$
U^{\prime \prime}(y)=f(U(y), z) \quad \forall y \in \mathbb{R}, \quad U(0)=\phi(z), \quad U^{\prime}(0)=-\sqrt{2 G(\phi(z), 1, z)} .
$$

Note that $U^{\prime 2}=2 G(U, 1, z)=2 \int_{1}^{U} f(s, z) d s$ on $\mathbb{R}, U^{\prime}<0$ on $(-\infty, 0]$, and $U(-\infty)=1$.

Now suppose $J(z)<0$. Then $\phi_{0}(z)=0<\phi(z)<\phi^{*}(z)<1$ and $G(v, 1, z)=$ $\int_{1}^{v} f(s, z) d s=-J(z)+\int_{0}^{v} f(s, z) d s \geq|J(z)|$ for all $v<\phi(z)$. Thus, for $T=$ $\int_{0}^{\phi(z)} \frac{1}{\sqrt{2 G(s, 1, z)}} d s, U(T)=0$ and $U^{\prime}<0$ on $(-\infty, T]$. It then follows from (23) that for some $\tau_{1}=z+\varepsilon[T+O(\varepsilon)], u\left(\tau_{1}\right)=0$ and $\dot{u}<0$ in $\left(\tau, \tau_{1}\right]$. This proves the assertion (c).

Next, we consider the case $J(z)>0$. In this case, we have $0<\phi^{*}(z)<\phi(z)<$ $1=\phi_{0}(z)$ and $F(v, z)=G(v, 1, z)=\int_{1}^{v} f(s, z) d s$ for all $v$. In the rest of the proof, we use $F(v, x)=\int_{1}^{v} f(s, x) d s$.

Setting $T_{1}=\int_{\phi^{*}(z)}^{\phi(z)} \frac{1}{\sqrt{2 F(s, z)}} d s$, we have $U\left(T_{1}\right)=\phi^{*}(z), U^{\prime}\left(T_{1}\right)=0, U^{\prime}<0$ in $\left(-\infty, T_{1}\right)$, and $U$ is even about $y=T_{1}$. From (23), there exists $\tau_{1}=z+\varepsilon\left[T_{1}+O(\varepsilon)\right]$ such that

$$
\dot{u}<0 \text { in }\left(\tau, \tau_{1}\right), \quad \dot{u}\left(\tau_{1}\right)=0, \quad u\left(\tau_{1}\right)=\phi^{*}(z)+O(\varepsilon), \quad \ddot{u}\left(\tau_{1}\right)>0 .
$$

Now we define

$$
\tau_{2}=\sup \left\{x>\tau_{1} \mid u-1<0<\dot{u} \text { in }\left(\tau_{1}, x\right)\right\} \in\left(\tau_{1}, \infty\right) \cup\{\infty\} .
$$


Then either $\dot{u}\left(\tau_{2}\right)=0$ or $u\left(\tau_{2}\right)=1$. Since $U(y)=1-O\left(e^{-\nu|y|}\right)$ and $U^{\prime}(y)>e^{-K y}$ for $y \gg 1$, we see from (23) that $\tau_{2}>z+\frac{1}{2 K+1} \varepsilon|\ln \varepsilon|$.

Now let $T=\sqrt{|\log \varepsilon|}$. Integrating $\frac{d}{d x}\left(\frac{1}{2} \varepsilon^{2} \dot{u}^{2}-F(u, x)\right)=-F_{x}$ over $\left(\tau, \tau_{2}\right)$ we obtain

$$
\frac{1}{2} \varepsilon^{2} \dot{u}^{2}\left(\tau_{2}\right)-F\left(u\left(\tau_{2}\right), \tau_{2}\right)=-F(u(\tau), \tau)-\left\{\int_{\tau}^{z-\varepsilon T}+\int_{z-\varepsilon T}^{z+\varepsilon T}+\int_{z+\varepsilon T}^{\tau_{2}}\right\} F_{x}(u(x), x) d x .
$$

Recall that $F(u(\tau), \tau)=O\left(e^{-\nu \delta / \varepsilon}\right)$. Also, using (23) we have

$$
\begin{aligned}
\int_{z-\varepsilon T}^{z+\varepsilon T} F_{x} d x & =\varepsilon \int_{-T}^{T}\left\{F_{x}(U(y), z)+O(1)(u-U)+O(\varepsilon y)\right\} d y \\
& =\varepsilon\left\{\int_{-T}^{T} F_{x}(U, z) d y+O(\varepsilon) e^{K T}\right\} \\
& =\varepsilon\left\{-2 S(z)+O\left(e^{-\nu T}\right)+O\left(\varepsilon e^{K T}\right)\right\}
\end{aligned}
$$

since $\int_{|y|>T}\left|F_{x}(U, z)\right| d y=O\left(e^{-\nu T}\right)$ and $\int_{\mathbb{R}} F_{x}(U(y), z) d y=2 \int_{\phi^{*}(z)}^{\phi_{0}(z)} \frac{F_{x}(s, z)}{\sqrt{2 F(s, z)}} d s=$ $-2 S(z)$.

In the interval $\left[z+\varepsilon T, \tau_{2}\right)$, we have $-\frac{d}{d x}\left(\varepsilon^{2} \dot{u}^{2}\right)=-2 \dot{u} f(u, x) \geq 2 \nu^{2}(1-u) \dot{u}$. Integrating over $\left[x, \tau_{2}\right]$ we then obtain, for $x \in\left[z+\varepsilon T, \tau_{2}\right)$,

$$
\varepsilon^{2} \dot{u}^{2}(x) \geq \varepsilon^{2} \dot{u}^{2}\left(\tau_{2}\right)+2 \nu^{2} \int_{u(x)}^{u\left(\tau_{2}\right)}(1-u) d u \geq \nu^{2}\left\{(1-u(x))^{2}-\left(1-u\left(\tau_{2}\right)\right)^{2}\right\} .
$$

Thus, $\varepsilon \dot{u}(x) \geq \nu \sqrt{(1-u(x))^{2}-\left(1-u\left(\tau_{2}\right)\right)^{2}}$. Consequently, since $\left|F_{x}(v, x)\right|=$ $O(1)(1-v)^{2}$, we have the estimate

$$
\begin{aligned}
\int_{z+\varepsilon T}^{\tau_{2}}\left|F_{x}\right| d x & =\int_{u(z+\varepsilon T)}^{u\left(\tau_{2}\right)} \frac{\varepsilon\left|F_{x}\right|}{\sqrt{\varepsilon^{2} \dot{u}^{2}}} d u \\
& \leq O(\varepsilon) \int_{u(z+\varepsilon T)}^{u\left(\tau_{2}\right)} \frac{(1-s)^{2}}{\sqrt{(1-s)^{2}-\left(1-u\left(\tau_{2}\right)\right)^{2}}} d s \\
& =O(\varepsilon)[1-u(z+\varepsilon T)]^{2}=O(\varepsilon)\left\{e^{-\nu T}+\varepsilon e^{K T}\right\} .
\end{aligned}
$$

In a similar manner, we can estimate $\int_{\tau}^{z-\varepsilon T}\left|F_{x}\right| d x$. Hence,

$$
\frac{1}{2} \varepsilon^{2} \dot{u}^{2}\left(\tau_{2}\right)-F\left(u\left(\tau_{2}\right), \tau_{2}\right)=\varepsilon\left\{2 S(z)+O\left(e^{-\nu \delta / \varepsilon}\right)+O\left(e^{-\nu T}\right)+\varepsilon e^{K T}\right\} .
$$

Now suppose, in addition to $J(z)>0$, that $S(z)>0$. Then the right-hand side of (24) is positive. As either $\dot{u}\left(\tau_{2}\right)=0$ or $F\left(u\left(\tau_{2}\right), \tau_{2}\right)=0$, we conclude that $\tau_{2}<\infty, \dot{u}\left(\tau_{2}\right)>0$, and $u\left(\tau_{2}\right)=1$. This proves assertion (b) of the lemma.

Finally we consider the case $J(z)>0>S(z)$. Then the right-hand side of (24) is negative. From the definition of $\tau_{2}$, we then conclude that $\tau_{2}<\infty, \dot{u}\left(\tau_{2}\right)=0$ and $u\left(\tau_{2}\right)<1$. Once we know the existence of $\tau_{2}$, we can then use the estimates (16) and (15) to conclude that $F\left(u\left(\tau_{2}\right), \tau_{2}\right)=-2 \varepsilon S(z)+O\left(\varepsilon^{2}|\ln \varepsilon|\right)$ and $\tau_{2}=z+O(\varepsilon|\ln \varepsilon|)$. Following the same argument, we can show that for each $i=3, \cdots, N$, there is a $\tau_{i}=z+O(\varepsilon|\ln \varepsilon|)$ such that $\dot{u}\left(\tau_{i}\right)=0$ and $F\left(u\left(\tau_{i}\right), \tau_{i}\right)=-i \varepsilon S(z)+O\left(\varepsilon^{2}|\ln \varepsilon|\right)$. This proves assertion (a), and also completes the proof of the lemma. 


\section{An induCtion ARgument}

Set $\xi_{0}=0$. For each $k \geq 0$ such that $\sigma_{k}>0$, we shall prove by induction the following.

Induction hypothesis. There exists an open interval $I_{k}$ such that for each $\alpha \in I_{k}$, $u(\cdot, \alpha)$ has at least $m:=\sum_{i=0}^{k} \sigma_{i}$ critical points in $(0, \infty), \tau_{m}(\alpha)>\xi_{k}+2 \delta$, and for each $i=0, \cdots, k$, there are exactly $\sigma_{i}$ critical points of $\dot{u}(\cdot, \alpha)$ in a $\sqrt{\varepsilon}$ neighborhood of $\xi_{i}$. In addition, $\tau\left(I_{k}\right):=\left\{\tau_{m}(\alpha) \mid \alpha \in I_{k}\right\}=\left(\xi_{k}+2 \delta, \infty\right)$. Furthermore, using $\bar{I}$ to denote the closure of a set $I$, we have $\bar{I}_{k} \subset I_{l}$ for all integer $l \in[0, k-1]$ such that $\sigma_{l}>0$.

Here the $\sqrt{\varepsilon}$ neighborhood of $\xi_{0}$ means the interval $[0, \sqrt{\varepsilon}]$. Note that by Lemma 5.1. the hypothesis implies that all $\tau_{i}(\alpha)$ and $z_{i+1 / 2}$ for $i=0, \cdots, m$ are continuous functions of $\alpha \in I_{k}$. In addition, $\tau_{m}(\alpha)$ approaches $\infty$ as $\alpha$ approaches one of the boundary points of $I_{k}$ and approaches $\tau_{m}=\xi_{k}+2 \delta$ at the other boundary point. Also, taking $\alpha \in \bar{I}_{k}$ such that $L=\tau_{m}(\alpha)$ we obtain the assertion of the theorem, without the boundary oscillation at $L$.

To start the induction, we need to distinguish two different cases: (i) $\sigma_{0}>0$, so the first cluster of oscillations is at the left boundary; and (ii) $\sigma_{0}=0$, so that the first cluster of oscillations is either interior or at the right boundary.

6.1. Left boundary oscillations. We start the proof of Theorem 4 by constructing the desired number, $\sigma:=\sigma_{0}>0$, of boundary oscillations at $x=0$. Working on $1-u$ if necessary, we assume that 0 is a local maximum. Set $\alpha_{0}=\phi(0)+\mu$ and define

$$
\alpha^{+}=\inf \left\{\alpha>\alpha_{0} \mid \text { There are fewer than } \sigma \text { critical points of } u(\cdot, \alpha) \text { in }(0, \infty)\right\} \text {. }
$$

Then $\alpha^{+} \leq 1$ since $\dot{u}>0$ on $(0, \infty)$ for every $\alpha>1$. When $\alpha=\alpha_{0}$, an easy phase plane argument shows that for sufficiently small $\varepsilon$ the solution $u$ of (22) has at least $N$ oscillations in $[0, \delta]$. Hence, $\alpha^{+} \in\left(\alpha_{0}, 1\right]$.

If $\alpha \in\left[\alpha_{0}, \alpha^{+}\right), u(\cdot, \alpha)$ has at least $\sigma$ critical points in $(0, \infty)$. Hence, by Lemma 5.1. $\tau_{i}(\cdot)$ and $z_{i+1 / 2}(\cdot)$, for all $i=0,1, \cdots, \sigma$, are continuous functions on $\left[\alpha_{0}, \alpha^{+}\right)$. Note that $\tau_{\sigma}\left(\alpha_{0}\right)<\delta$ and $\lim _{\alpha \nearrow \alpha^{+}} \tau_{\sigma}(\alpha)=\infty$.

Set

$$
\alpha^{-}=\max \left\{\alpha \in\left[\alpha_{0}, \alpha^{+}\right) \mid \tau_{\sigma}(\alpha)=2 \delta\right\}, \quad I_{0}=\left(\alpha^{-}, \alpha^{+}\right)
$$

Then $\tau\left(I_{0}\right):=\left\{\tau_{\sigma}(\alpha) \mid \alpha \in I_{0}\right\}=(2 \delta, \infty)$ and $\tau_{\sigma}\left(\alpha^{-}\right)=2 \delta$.

We claim that $z_{\sigma-1 / 2}(\alpha) \leq \sqrt{\varepsilon}$ for all $\alpha \in I_{0}$ so that the induction hypothesis for $k=0$ holds (in the case $\sigma_{0}>0$ ). By Theorem 11, it suffices to show that $z_{\sigma-1 / 2}(\alpha)<\delta$ for all $\alpha \in\left[\alpha_{0}, \alpha^{+}\right)$. Suppose this is not true. Then by continuity there is an $\hat{\alpha} \in\left(\alpha_{0}, \alpha^{+}\right)$such that $z_{\sigma-1 / 2}(\hat{\alpha})=\delta$. Since $J S \neq 0$ in $(0,6 \delta]$, applying Theorem 1 on interval $[a, b]=\left[0, \tau_{\sigma}(\hat{\alpha})\right]$, we then conclude that $\tau_{\sigma}(\hat{\alpha})<\delta+\sqrt{\varepsilon}<2 \delta$. Hence, $u(\cdot, \hat{\alpha})$ admits boundary spikes at $b$.

From Theorem 1(i), the fact that the desired solution has $\sigma$ boundary oscillations implies that $\tau_{\sigma}$ is a local maxima if $\phi_{0}(0+)=1$ and is a local minimum if $\phi_{0}(0+)=$ 0 . As $\phi_{0}(0+)=\phi_{0}(x)$ for all $x \in(0,2 \delta]$, we see that the boundary spike at $b$ cannot be a half spike. Thus $\sigma \geq 2$. Consequently, by Theorem 1(iii) we have $J S<0$ in $[b-\delta, b]$. On the other hand, the existence of a boundary cluster of $\sigma_{0}$ spikes at $\xi_{0}=0$ implies $J S>0$ in $(0, \delta]$. As $b<2 \delta$, we obtain a contradiction. This contradiction shows that the claim is true. 
6.2. The first interior oscillation without boundary oscillations. In this section we consider the case $\sigma_{0}=0$. For convenience, we delete from the list $\left\{\xi_{i}\right\}$ those $\xi_{i}$ for which $\sigma_{i}=0$. Then $\sigma_{i}>0$ for all $i \geq 1$. Note that $\left\{\xi_{i}\right\}_{i=1}^{\infty}$ becomes a subset of $Z$.

We now prove the induction for $k=1$ with $\sigma_{0}=0$. We set $\sigma:=\sigma_{1}>0$. Again, we assume 0 is a local maximum for the desired solution. By Lemma 5.1 $z_{1 / 2}(\cdot)$ exists and is continuous on $\left[\alpha_{0}, 1\right)$. Note that $\lim _{\alpha / 1} z_{1 / 2}(\alpha)=\infty$ and $z_{1 / 2}\left(\alpha_{0}\right)<\tau_{1}\left(\alpha_{0}\right)=O(\varepsilon)$. We define

$$
\begin{gathered}
\beta^{-}=\max \left\{\alpha \in\left(\alpha_{0}, 1\right) \mid z_{1 / 2}(\alpha)=\xi_{1}-\delta\right\}, \\
\beta^{+}=\min \left\{\alpha>\beta^{-} \mid z_{1 / 2}(\alpha)=\xi_{1}+\delta\right\} .
\end{gathered}
$$

Then $z_{1 / 2}(\alpha) \in\left(\xi_{1}-\delta, \xi_{1}+\delta\right)$ for all $\alpha \in\left(\beta_{1}, \beta_{2}\right), z_{1 / 2}\left(\beta^{ \pm}\right)=\xi_{1} \pm \delta$.

Since $\xi_{1} \in Z$, there are only three possibilities:

(i) $J\left(\xi_{1}\right)<0$.

(ii) Either $J\left(\xi_{1}\right)=0>\dot{J}\left(\xi_{1}\right)$ or $\left\{J\left(\xi_{1}\right)>0\right.$ and $\left.S\left(\xi_{1}\right)=0<\dot{S}\left(\xi_{1}\right)\right\}$.

(iii) Either $J\left(\xi_{1}\right)=0<\dot{J}\left(\xi_{1}\right)$ or $\left\{J\left(\xi_{1}\right)>0\right.$ and $\left.S\left(\xi_{1}\right)=0>\dot{S}\left(\xi_{1}\right)\right\}$.

We claim that (i) is impossible. Indeed, suppose it were true. Then $\phi_{0}=0$ in $\left[\xi_{1}-2 \delta, \xi_{1}+2 \delta\right]$, so that there can only be upward spikes near $\xi_{1}$. Since the solution we are looking for has no other boundary or interior oscillations before $\xi_{1}, 0=\tau_{0}$ must be a local minimum, contradicting our assumption that 0 is a local maximum.

Next we consider case (ii). Then in $\left[\xi_{1}-2 \delta, \xi_{1}\right), J>0$ and $\phi_{0}=1$. Using (77) if necessary we see that $J S<0$ in $\left[\xi_{1}-2 \delta, \xi_{1}\right)$ and $J S>0$ in $\left(\xi_{1}, \xi_{1}+2 \delta\right]$. Hence, Theorem 1 allows either any odd number of layers near $\xi_{1}$ or an arbitrary integer number of spikes near $\xi_{1}$.

Applying Lemma 5.2(a) for $\tau=0, z=\xi_{1}-\delta$, and $u=u\left(\cdot, \beta^{-}\right)$, we see that $u\left(\cdot, \beta^{-}\right)$has at least $N$ oscillations in $\left[\xi_{1}-\delta, \xi_{1}\right)$.

Note that $\sigma \geq 1$ if $J\left(\xi_{1}\right)=0$ and $\sigma \geq 2$ if $S\left(\xi_{1}\right)=0$. For $u=u\left(\cdot, \beta^{+}\right), \tau=0$, and $z=\xi_{1}+\delta$, we can apply Lemma $\left[5.2(\mathrm{~b})\right.$ in the case $S\left(\xi_{1}\right)=0$ and part (c) in the case $J\left(\xi_{1}\right)=0$ to conclude that $u\left(\cdot, \beta^{+}\right)$has fewer than $\sigma$ critical points in $(0, \infty)$.

We define

$$
\alpha^{+}=\inf \left\{\alpha>\beta^{-} \mid \text {There are fewer than } \sigma \text { critical points of } u(\cdot, \alpha) \text { in }(0, \infty)\right\} \text {. }
$$

Then $\alpha^{+} \in\left(\beta^{-}, \beta^{+}\right)$. Also, for each $\alpha \in\left[\beta^{-}, \alpha^{+}\right), u(\cdot, \alpha)$ has at least $\sigma$ critical points in $(0, \infty)$ and $\tau_{\sigma}(\cdot)$ is a continuous function over $\left[\beta^{-}, \alpha^{+}\right)$. Further, $\lim _{\alpha / \alpha^{+}} \tau_{\sigma}(\alpha)=\infty$. Define

$$
\alpha^{-}=\max \left\{\alpha<\alpha^{+} \mid \tau_{\sigma}(\alpha)=\xi_{1}+2 \delta\right\}, \quad I_{1}=\left(\alpha^{-}, \alpha^{+}\right) .
$$

Then $\tau_{\sigma}\left(\alpha^{-}\right)=\xi_{1}+2 \delta$ and $\left\{\tau_{\sigma}(\alpha) \mid \alpha \in I_{1}\right\}=\left(\xi_{1}+\delta, \infty\right)$.

Since $I_{1} \subset\left(\beta^{-}, \beta^{+}\right), z_{1 / 2}(\alpha)>\xi_{1}-\delta$ for all $\alpha \in I_{1}$. Since $J S>0$ in $\left(\xi_{1}, \xi_{1}+2 \delta\right]$, following a similar argument as for the case $\sigma_{0}>0$ we can show that $z_{\sigma-1 / 2}(\alpha)<$ $\xi_{1}+\delta$ for all $\alpha \in I_{1}$. An application of Theorem 1 for $u(\cdot, \alpha)$ in $\left(0, \tau_{\sigma}(\alpha)\right)$ then gives us $\left|z_{i-1 / 2}(\alpha)-\xi_{1}\right|<\sqrt{\varepsilon}$ for all $i=1, \cdots, \sigma$. This validates the induction hypothesis for $k=1$ for the case (ii).

Finally we consider case (iii). Since $0=\tau_{0}$ is a local maximum and $\xi_{1}$ is the first desired place for an oscillation cluster, Theorem 1 implies that only a single layer ( $\sigma=1$ when $\left.J\left(\xi_{1}\right)=0\right)$ or a single spike $\left(\sigma=2\right.$ when $\left.S\left(\xi_{1}\right)=0\right)$ is possible. 
We define

$\alpha^{-}=\sup \left\{\alpha<\beta^{+} \mid\right.$There are fewer than $\sigma$ critical points of $u(\cdot, \alpha)$ in $\left.(0, \infty)\right\}$.

With $\tau=0, z=\xi_{1}-\delta$, and $u=u\left(\cdot, \beta^{-}\right)$, we can apply Lemma 5.2 (b) for the case $S\left(\xi_{1}\right)=0$ and (c) for the case $J\left(\xi_{1}\right)=0$ to conclude that $u\left(\cdot, \beta^{-}\right)$has fewer than $\sigma$ critical points in $(0, \infty)$. Hence, $\alpha^{-}>\beta^{-}$.

Next, we show that $\alpha^{-}<\beta^{+}$. For this, we set $\tau=0, z=\xi_{1}+\delta$ and consider $u\left(\cdot, \beta^{+}\right.$). If $J\left(\xi_{1}\right)=0$ (so that $\sigma=1$ ), we see from (iii) that $J(z)>0$ and from (77) that $S(z)>0$, so that Lemma 5.2(b) implies that $u\left(\cdot, \beta^{+}\right)$has a critical point. If $S\left(\xi_{1}\right)=0$, we see from (iii) that $J(z)>0$ and $S(z)<0$, and Lemma 5.2 (a) implies that $u\left(\cdot, \beta^{+}\right)$has at least $N$ critical points in $[z, z+\delta)$. In conclusion $\alpha^{-}<\beta^{+}$.

Thus $\alpha^{-} \in\left(\beta^{-}, \beta^{+}\right)$, and $\tau_{\sigma}(\alpha)$ exists and is a continuous function on $\left(\alpha^{-}, \beta^{+}\right]$. In addition $\lim _{\alpha \backslash \alpha^{-}} \tau_{\sigma}(\alpha)=\infty$. Set

$$
\alpha^{+}=\min \left\{\alpha>\alpha^{-} \mid \tau_{\sigma}(\alpha)=\xi_{1}+2 \delta\right\}, \quad I_{1}=\left(\alpha^{-}, \alpha^{+}\right) .
$$

Then $\tau_{\sigma}\left(\alpha^{+}\right)=\xi_{1}+2 \delta$ and $\tau_{\sigma}\left(I_{1}\right)=\left(\xi_{1}+2 \delta, \infty\right)$.

Since $I_{1} \subset\left(\beta^{-}, \beta^{+}\right),\left|z_{1 / 2}-\xi_{1}\right| \leq \delta$ for all $\alpha \in I_{1}$. If $\sigma=2$, then $\tau_{1}$ is the peak of a spike, so $z_{3 / 2}-z_{1 / 2}=O(\varepsilon)$. After applying Theorem 1, we then conclude that $z_{i-1 / 2}(\alpha)$ is within an $\sqrt{\varepsilon}$ neighborhood of $\xi_{1}$ for $i=1$ if $\sigma=1$ and for $i=1,2$ if $\sigma=2$.

6.3. The induction from $k=\ell$ to $k=\ell+1$. Let $\ell \geq 0$ be an arbitrary integer. Assume that the induction hypothesis holds for $k=\ell$. We now show that it also holds for $k=\ell+1$. As mentioned earlier, we delete from $\left\{\xi_{i}\right\}$ those $\xi_{i}$ for which $\sigma_{i}=0$. Then $\sigma_{\ell}>0$. We write $m=\sum_{i=0}^{\ell} \sigma_{i}$, and $\sigma=\sigma_{\ell+1}$. By working on $1-u$ if necessary, we assume that $\tau_{m}$ is a local maximum of the desired solution.

Let $\hat{\alpha} \in \partial I_{\ell}$ be that endpoint of the interval $I_{\ell}$ satisfying $\tau_{m}(\hat{\alpha})=T:=$ $\xi_{\ell}+2 \delta$. Let $\hat{u}=u(\cdot, \hat{\alpha})$. Since $\hat{u}$ attains a local maximum at $T=\tau_{m}(\alpha)$, $z_{m+1 / 2}(\hat{\alpha})$ is finite. We now estimate $z_{m+1 / 2}(\hat{\alpha})$. We have $\sigma_{\ell}>0$ and $\mid z_{m-1 / 2}(\hat{\alpha})-$ $\xi_{\ell} \mid<\sqrt{\varepsilon}$. By (9) with $i=m, z_{m+1 / 2}(\hat{\alpha})=\xi_{\ell}+4 \delta+O\left(\delta^{2}\right)$. As $z_{m+1 / 2}(\alpha)>$ $\tau_{m}(\alpha) \rightarrow \infty$ as $\alpha$ approaches the other boundary point of $I_{\ell}$, there exists an interval $\left(\beta^{-}, \beta^{+}\right) \subset I_{\ell}$ such that $z_{m+1 / 2}(\alpha) \in\left(\xi_{\ell+1}-\delta, \xi_{\ell+1}+\delta\right)$ for all $\alpha \in\left(\beta^{-}, \beta^{+}\right)$, and $\min \left\{z_{m+1 / 2}\left(\beta^{-}\right), z_{m+1 / 2}\left(\beta^{+}\right)\right\}=\xi_{\ell+1}-\delta$ and $\max \left\{z_{m+1 / 2}\left(\beta^{-}\right), z_{m+1 / 2}\left(\beta^{+}\right)\right\}=$ $\xi_{\ell+1}+\delta$.

From here, we can follow a similar argument as in the preceding subsection to show that the hypothesis for $k=\ell+1$ is valid, thereby completing the induction.

6.4. Oscillations at the right boundary. Finally, we attach $\sigma \geq 1$ oscillations at the right boundary $L$. For simplicity, we assume that either $L \in Z$ or $\operatorname{dist}(L, Z) \geq$ $6 \delta$.

If the desired solution has no cluster of oscillations except at the right boundary, then by reversing the $x$-axis, we can use the proof for the case $\sigma_{0}>0$ to finish the proof. Hence, we assume that there are other clusters of oscillations.

We assume that $\xi_{k}$ is the last point of cluster of oscillation before $L$. Then $\xi_{k} \leq L-6 \delta$. Set $m=\sum_{i=0}^{k} \rho_{k}$. By working on $1-u$ if necessary, we can assume that $\tau_{m}$ is a point of local maximum of the solution we are looking for. In order to attach at least a boundary oscillation at $L$, it is necessary, by Theorem 10 to have $\phi_{0}(x)=1$ for all $x \in[L-\delta, L)$, i.e., $J(x)>0$ in $[L-\delta, L)$. Following a similar argument as in the preceding subsection, we can find $\left(\beta^{-}, \beta^{+}\right) \in I_{k}$ such that $z_{m+1 / 2}(\cdot)$ maps $\left(\beta^{-}, \beta^{+}\right)$onto $(L-\delta, L+\delta)$. For definiteness, we assume that 
$z_{m+1 / 2}\left(\beta^{\mp}\right)=L \mp \delta$ (the other situation $z_{m+1 / 2}\left(\beta^{\mp}\right)=L \pm \delta$ can be similarly treated). There are two possibilities: (i) $S(L-\delta)<0$ and (ii) $S(L-\delta)>0$.

Suppose (i) $S(L-\delta)<0$. Since $J>0$ in $[L-\delta, L)$ and $\tau=\tau_{m}\left(\beta^{-}\right)=\xi_{k}+O(\sqrt{\varepsilon})$ is a local maximum of $u\left(\cdot, \beta^{-}\right)$, we apply Lemma 5.2 (a) with $z=z_{m+1 / 2}\left(\beta^{-}\right)=L-\delta$ to conclude that $u\left(\cdot, \beta^{-}\right)$has at least $N$ critical points in $[z, z+\sqrt{\varepsilon}$. Hence, $\tau_{m+\sigma}\left(\beta^{-}\right)<L$. Increasing $\alpha$ we then conclude there exists $\alpha^{*} \in\left(\beta^{-}, \beta^{+}\right)$such that $\tau_{m+\sigma}\left(\alpha^{*}\right)=L$. As $\alpha^{*} \in\left(\beta^{-}, \beta^{+}\right), z_{m+1 / 2}\left(\alpha^{*}\right)>L-\delta$ so that $z_{m+i-1 / 2} \in(L-\delta, L)$ for all $i=1, \cdots, \sigma$. Applying Theorem 1 to $u\left(\cdot, \alpha^{*}\right)$, we see that it is the solution we are looking for.

Next suppose (ii) $S(L-\delta)>0$. Then $J S>0$ in $[L-\delta, L)$ so that, by Theorem 1 (iii), the boundary oscillation attached is a half spike. Now consider $u\left(\cdot, \beta^{-}\right)$. Since $\phi^{*}(L-\delta) \in(0, \phi(L-\delta))$, we can derive from Lemma 5.2 (b) that $\tau_{m+1}\left(\beta^{-}\right)=$ $z_{m+1 / 2}\left(\beta^{-}\right)+O(\varepsilon)$ exists and $u\left(\tau_{m+1}(\beta), \beta^{-}\right)=\phi^{*}(L-\delta)+O(\varepsilon)$ is the peak of a spike. Gradually increasing $\alpha$ from $\beta^{-}$to $\beta^{+}$, we then see that there is an $\alpha^{*}$ such that $\tau_{m+1}\left(\alpha^{*}\right)=L$. As $\alpha^{*} \in\left(\beta^{-}, \beta^{+}\right) \subset I_{k}$, we can apply Theorem 1 to show that $u\left(\cdot, \alpha^{*}\right)$ is the solution we desired. This completes the proof of Theorem 4 in the case $k<\infty$.

Finally, if $k$ is $\infty$ in the theorem, then $u(\cdot, \alpha)$ with $\alpha \in \bigcap_{i=1}^{\infty} \bar{I}_{i}$ is the desired solution. This completes the proof of Theorem 4 .

\section{Part III: The Morse index}

\section{The Morse IndeX AND METhod OF STUdy}

7.1. The Morse index. We now study the eigenvalue problem, for $(\lambda, \psi)$,

$$
-\varepsilon^{2} \ddot{\psi}+f_{u}(u, x) \psi=\lambda \psi \text { in } \mathrm{I}, \quad \dot{\psi}=0 \text { on } \partial \mathrm{I},
$$

where we assume that $\mathrm{I}=(a, b)$ is a bounded interval. Since all eigenvalues are real and simple, we denote them, in order from small to large, by $\lambda_{1}, \lambda_{2}, \cdots$.

Definition 2. The Morse index of a solution $u$ to (1) is defined to be the number of non-positive eigenvalues to (25).

Definition 3. A stable layer is an interval $\left(\tau_{l}, \tau_{l+1}\right)$ with an interior cluster of a single transition layer satisfying $\dot{u}_{l+1 / 2} \dot{J}\left(z_{l+1 / 2}\right)<0$, or equivalently, $0 \leq l \leq n-1$,

$$
z_{l-1 / 2}+\sqrt{\varepsilon}<z_{l+1 / 2}<z_{l+3 / 2}-\sqrt{\varepsilon}, \quad \dot{u}_{l+1 / 2} \dot{J}\left(z_{l+1 / 2}\right)<0 .
$$

A semi-stable spike is an interval $\left(\tau_{i-1}, \tau_{i+1}\right)$ with an interior cluster of a single spike satisfying $J\left(\tau_{i}\right) \dot{S}\left(\tau_{i}\right)<0$, or equivalently, $1 \leq i \leq n-1$,

$$
z_{i+1 / 2}-z_{i-1 / 2} \leq \sqrt{\varepsilon}<\min \left\{z_{i-1 / 2}-z_{i-3 / 2}, z_{i+3 / 2}-z_{i+1 / 2}\right\}, \quad J\left(\tau_{i}\right) \dot{S}\left(\tau_{i}\right)<0 .
$$

Theorem 5. Assume (2), (4) and (5). Let $u \in \mathcal{S}_{N, \varepsilon}$ with $\varepsilon$ sufficiently small. Then the Morse index associated with $u$ is equal to

$$
n-n_{s l}-n_{s s},
$$

where $n$ is the total number of oscillations, $n_{s l}$ is the total number of stable layers and $n_{s s}$ is the total numbers of semi-stable spikes. 
7.2. A criterion. We will use the following lemma to study the Morse index for our problem.

Lemma 7.1. Let $w$ be the solution to

$$
\varepsilon^{2} \ddot{w}=f_{u} w \text { in } \mathrm{I}, \quad w(a)=1, \quad \dot{w}(a)=0 .
$$

(1) If $\dot{w}(b)>0$ and there are no roots of $w=0$ in $(a, b)$, then $\lambda_{1}>0$.

(2) If $\dot{w}(b) \neq 0$ and for some $k \geq 1$, either $\{w=0$ has $k-1$ roots in $(a, b)$ and $w(b) \dot{w}(b) \leq 0\}$ or $\{w=0$ has $k$ roots in $(a, b)$ and $w(b) \dot{w}(b)>0\}$, then $\lambda_{k}<0<\lambda_{k+1}$.

(3) If $\dot{w}(b)=0$ and $w=0$ has $k-1$ roots in $(a, b)$, then $\lambda_{k}=0$.

Proof. Let $\psi(\cdot, \lambda)$ be the solution to $\varepsilon^{2} \ddot{\psi}=\left(f_{u}-\lambda\right) \psi$ with initial values $\psi(a, \lambda)=1$ and $\dot{\psi}(a, \lambda)=0$. Referring to the phase plane for $(\psi, \varepsilon \dot{\psi})$ we let $\theta(x, \lambda)=\arctan \frac{\varepsilon \dot{\psi}}{\psi}$. Then

$$
\varepsilon \dot{\theta}=\left(f_{u}-\lambda\right) \cos ^{2} \theta-\sin ^{2} \theta \text { in I, } \quad \theta(a, \lambda)=0 .
$$

It is easy to check that $\left.\dot{\theta}\right|_{\theta=\frac{\pi}{2}+\ell \pi}<0$ for all integers $\ell$ and that $\frac{\partial}{\partial \lambda} \theta<0$ for all $x \in \overline{\mathrm{I}}$ and $\lambda \in \mathbb{R}$. Hence, for each integer $\ell \geq 1, \theta\left(b, \lambda_{\ell}\right)+(\ell-1) \pi=0, \theta(b, \lambda)+(\ell-1) \pi<0$ if $\lambda_{\ell}<\lambda$, and $\theta(b, \lambda)+(\ell-1) \pi>0$ if $\lambda<\lambda_{\ell}$. Note that $\theta(x, 0)=\arctan \frac{\varepsilon \dot{w}}{w}$.

Now in case (1), we have $\theta(b, 0)>0$ so that $0<\lambda_{1}$. In case (2), we can see that $\theta(b, 0)+(k-1) \pi \in(-\pi, 0)$, so that $\lambda_{k}<0<\lambda_{k+1}$. In case (3), there holds $\theta(b, 0)+(k-1) \pi=0$ so $\lambda_{k}=0$.

7.3. Fundamental solution matrices. To study the solution $w$ to (26), we will often regard (26) as a system of two first order odes. Hence, we introduce vector notation

$$
\mathbf{w}=\left[\begin{array}{c}
w \\
\dot{w}
\end{array}\right], \quad \tilde{\mathbf{w}}=\left[\begin{array}{c}
w \\
\varepsilon \dot{w}
\end{array}\right]=\mathbf{P} \mathbf{w}, \quad \mathbf{P}=\left[\begin{array}{ll}
1 & 0 \\
0 & \varepsilon
\end{array}\right] .
$$

Definition 4. A $2 \times 2$ matrix function $\Psi(x, y)$ is called a fundamental solution matrix if

$$
\dot{\Psi}(\cdot, y)=\mathbf{A} \Psi,\left.\quad \Psi\right|_{x=y}=\mathbf{I}, \quad \text { where } \quad \mathbf{A}:=\left[\begin{array}{cc}
0 & 1 \\
\varepsilon^{-2} f_{u} & 0
\end{array}\right] .
$$

Note that for all $x, \tau \in[a, b]$,

$$
\mathbf{w}(x)=\Psi(x, \tau) \mathbf{w}(\tau), \quad \tilde{\mathbf{w}}(x)=\mathbf{P} \Psi(x, \tau) \mathbf{P}^{-1} \tilde{\mathbf{w}}(\tau) .
$$

To find $\Psi(x, a)$, we will decompose $(a, b)$ into sub-intervals and use the following properties for fundamental solution matrices:

$$
\Psi(x, y)=\Psi^{-1}(y, x)=\Psi(x, \tau) \Psi(\tau, y) \quad \forall x, y, \tau \in \overline{\mathrm{I}} .
$$

In our application, $\tau$ will be a critical point of $u$. Hence, we define two linearly independent solutions $\omega, \omega_{1}$, piecewise, as the fundamental solution set in each interval $\left[z_{i-1 / 2}+0, z_{i+1 / 2}-0\right]$ :

$$
\left\{\begin{array}{l}
\varepsilon^{2} \ddot{\omega}_{1}=f_{u} \omega_{1}, \quad \varepsilon^{2} \ddot{\omega}=f_{u} \omega \quad \text { in } \mathrm{I}_{i}:=\left[z_{i-1 / 2}+0, z_{i+1 / 2}-0\right], \quad \forall i . \\
\omega_{1}\left(\tau_{i}\right)=\dot{\omega}\left(\tau_{i}\right)=1, \quad \dot{\omega}_{1}\left(\tau_{i}\right)=\omega\left(\tau_{i}\right)=0
\end{array}\right.
$$

One of the keys here is to study the jumps of $\frac{\dot{\omega}}{\omega}$ and $\frac{\omega_{1}}{\omega}$ across $z_{i-1 / 2}$ for all $i$. 
Since $\omega_{1} \dot{\omega}-\dot{\omega}_{1} \omega \equiv 1$ and $\Psi\left(\tau_{i}, x\right)=\Psi^{-1}\left(x, \tau_{i}\right)$,

$$
\begin{aligned}
& \Psi\left(x, \tau_{i}\right)=\left[\begin{array}{cc}
\omega_{1} & \omega \\
\dot{\omega} & \dot{\omega}
\end{array}\right]=\omega\left\{\left[\begin{array}{c}
1 \\
\frac{\dot{\omega}}{\omega}
\end{array}\right]\left[\begin{array}{ll}
\frac{\omega_{1}}{\omega} & 1
\end{array}\right]-\frac{1}{\omega^{2}}\left[\begin{array}{ll}
0 & 0 \\
1 & 0
\end{array}\right]\right\}, \\
& \Psi\left(\tau_{i}, x\right)=\left[\begin{array}{cc}
\dot{\omega} & -\omega \\
-\dot{\omega}_{1} & \omega_{1}
\end{array}\right]=\omega\left\{\frac{1}{\omega^{2}}\left[\begin{array}{ll}
0 & 0 \\
1 & 0
\end{array}\right]-\left[\begin{array}{c}
1 \\
-\frac{\omega_{1}}{\omega}
\end{array}\right]\left[\begin{array}{ll}
-\frac{\dot{\omega}}{\omega} & 1
\end{array}\right]\right\} .
\end{aligned}
$$

As one will see below, $\omega$ can be well approximated by $\dot{u} / \ddot{u}\left(\tau_{i}\right)$. Also both $\frac{\dot{\omega}}{\omega}$ and $\frac{\varepsilon \omega_{1}}{\omega}$ can be accurately estimated and are of order one at $x=z_{i \pm 1 / 2}$. Hence, we introduce

$$
\begin{array}{rr}
\rho(x)=\frac{\dot{\omega}(x)}{\omega(x)}, & \gamma(x)=\frac{\varepsilon \omega_{1}(x)}{\omega(x)}, \quad \mathbf{e}_{1}=\left[\begin{array}{l}
1 \\
0
\end{array}\right], \quad \mathbf{e}_{2}=\left[\begin{array}{l}
0 \\
1
\end{array}\right], \\
\mathbf{n}=\left[\begin{array}{l}
1 \\
\rho
\end{array}\right], \quad \mathbf{n}^{\perp}=\left[\begin{array}{c}
-\rho \\
1
\end{array}\right], \quad \mathbf{t}=\left[\begin{array}{l}
\gamma \\
1
\end{array}\right], \quad \mathbf{t}^{\perp}=\left[\begin{array}{c}
1 \\
-\gamma
\end{array}\right] .
\end{array}
$$

We use the vector notation $\mathbf{a} \otimes \mathbf{b}=\mathbf{a b}^{T}$, where $\mathbf{a}$ and $\mathbf{b}$ are column vectors and ${ }^{T}$ denotes transpose. Thus $\mathbf{a} \otimes \mathbf{b}$ is a $2 \times 2$ matrix. The formula $(\mathbf{a} \otimes \mathbf{b})(\mathbf{c} \otimes \mathbf{d})=$ $\mathbf{b}^{T} \mathbf{c}(\mathbf{a} \otimes \mathbf{b})$ will be useful. We can write

$$
\begin{aligned}
& \Psi\left(x, \tau_{i}\right) \mathbf{P}^{-1}=\frac{\omega}{\varepsilon}\left\{\mathbf{n} \otimes \mathbf{t}-\frac{\varepsilon}{\omega^{2}} \mathbf{e}_{2} \otimes \mathbf{e}_{1}\right\}, \\
& \mathbf{P} \Psi\left(\tau_{i}, x\right)=\omega\left\{\frac{\varepsilon}{\omega^{2}} \mathbf{e}_{2} \otimes \mathbf{e}_{1}-\mathbf{t}^{\perp} \otimes \mathbf{n}^{\perp}\right\} .
\end{aligned}
$$

To study transition layers, we will need the matrix $\mathbf{P} \Psi\left(\tau_{i+1}, \tau_{i}\right) \mathbf{P}^{-1}$. In computing this we make use of the formulas $\mathbf{e}_{1}^{\top} \cdot \mathbf{n}_{-}=1,\left(\mathbf{n}_{+}^{\perp}\right)^{\top} \cdot \mathbf{n}_{-}=\rho_{-}-\rho_{+}$, $\left(\mathbf{n}_{+}^{\perp}\right)^{\top} \cdot \mathbf{e}_{2}=1$ and $\mathbf{e}_{1}^{\top} \cdot \mathbf{e}_{2}=0$. Letting $\omega_{ \pm}=\omega\left(z_{i+1 / 2} \pm 0\right)$, we find that

$$
\begin{aligned}
\mathbf{P} \Psi\left(\tau_{i+1}, \tau_{i}\right) \mathbf{P}^{-1} & =\mathbf{P} \Psi\left(\tau_{i+1}, z_{i+1 / 2}\right) \Psi\left(z_{i+1 / 2}, \tau_{i}\right) \mathbf{P}^{-1} \\
& =\frac{\omega_{+} \omega_{-}}{\varepsilon}\left\{\mathbf{t}_{+}^{\perp} \otimes \mathbf{t}_{-} \llbracket \rho \rrbracket_{-}^{+}+\mathbf{t}_{+}^{\perp} \otimes \mathbf{e}_{1} \frac{\varepsilon}{\omega_{-}^{2}}+\frac{\varepsilon}{\omega_{+}^{2}} \mathbf{e}_{2} \otimes \mathbf{t}_{-}\right\},
\end{aligned}
$$

where $\llbracket \rho \rrbracket_{-}^{+}=\rho_{+}-\rho_{-}$stands for the jump of $\rho$ across $z_{i \pm 1 / 2}$.

For spikes, we will need $\mathbf{P} \Psi\left(\tau_{i+1}, \tau_{i-1}\right) \mathbf{P}^{-1}$, where $\tau_{i}$ is the peak. Let $\omega_{R \pm}=$ $\omega\left(z_{i+1 / 2} \pm 0\right)$ and $\omega_{L \pm}=\omega\left(z_{i-1 / 2} \pm 0\right)$. Then

$$
\begin{aligned}
\mathbf{P \Psi}\left(\tau_{i+1}, \tau_{i-1}\right) \mathbf{P}^{-1} & =M_{i}\left\{\mathbf{t}_{R+}^{\perp} \otimes\left(a_{11} \mathbf{t}_{L-}+a_{12} \mathbf{e}_{1}\right)+\mathbf{e}_{2} \otimes\left(a_{21} \mathbf{t}_{L-}+a_{22} \mathbf{e}_{1}\right)\right\}, \\
M_{i} & =\frac{\omega_{R+} \omega_{R-} \omega_{L+} \omega_{L-}}{\varepsilon^{2}}, \\
a_{11} & =\llbracket \rho \rrbracket_{R-}^{R+} \llbracket \gamma \rrbracket_{L+}^{R-} \llbracket \rho \rrbracket_{L-}^{L+}+\frac{\varepsilon}{\omega_{L+}^{2}} \llbracket \rho \rrbracket_{R-}^{R+}+\frac{\varepsilon}{\omega_{R-}^{2}} \llbracket \rho \rrbracket_{L-}^{L+}, \\
a_{12} & =\frac{\varepsilon}{\omega_{L-}^{2}}\left\{\frac{\varepsilon}{\omega_{R-}^{2}}+\llbracket \rho \rrbracket_{R-}^{R+} \llbracket \gamma \rrbracket_{L+}^{R-}\right\}, \\
a_{21} & =\frac{\varepsilon}{\omega_{R+}^{2}}\left\{\frac{\varepsilon}{\omega_{L+}^{2}}+\llbracket \rho \rrbracket_{L-}^{L+} \llbracket \gamma \rrbracket_{L+}^{R-}\right\}, \\
a_{22} & =\frac{\varepsilon^{2}}{\omega_{L-}^{2} \omega_{R+}^{2}} \llbracket \gamma \rrbracket_{L+}^{R-} .
\end{aligned}
$$

As we will see, at the first spike of an interior cluster $a_{12}=O\left(e^{-1 / \sqrt{\varepsilon}}\right)$, so the sign of $w$ at $\tau_{i+1}$ is determined by $a_{11}$, the most difficult function to estimate. In the end, we will show that $a_{11} \propto \mathrm{H}\left(J\left(\tau_{i}\right)\right) \dot{S}\left(\tau_{i}\right)\left(g_{1} \propto g_{2}\right.$ means that $\frac{1}{C} \leq \frac{g_{1}}{g_{2}} \leq C$ for some positive constant $C$ independent of $\varepsilon$ ).

At a generic critical point $\tau_{i}$ of $u$, there are the following possibilities:

(i) $f_{i}^{2} \propto 1$ and $F_{i}=O(\varepsilon)\left(\right.$ or $\left.O\left(\varepsilon^{2}|\ln \varepsilon|\right)\right)$, i.e., $\tau_{i}$ is the peak of a spike; 
(ii) $f_{i}^{2} \propto \varepsilon|\ln \varepsilon|$, i.e., $\tau_{i}$ is a valley within a cluster of transition layers;

(iii) $f_{i}^{2} \propto \varepsilon$, i.e., $\tau_{i}$ is the valley of neighboring boundary cluster of spikes near $S \neq 0$.

(iv) $f_{i}^{2} \propto \varepsilon^{2}|\ln \varepsilon|$, i.e., $\tau_{i}$ is the valley of neighboring spikes in a cluster near $S=0$

(v) $f_{i}^{2}=O\left(e^{-1 / \sqrt{\varepsilon}}\right)$, i.e., $\tau_{i}$ is away from transition layers and spikes.

\section{The FUndamental SOlUtion SeT}

In this section, we study the fundamental solution set $\left(\omega_{1}, \omega\right)$ defined in (27).

\subsection{The function $\omega$.}

Lemma 8.1. Let $\omega$ be defined as in (27). Then for every $i$ there is a $C^{2}$ positive function $c$ on $\left[z_{i-1 / 2}+0, z_{i+1 / 2}-0\right]$ such that in this interval,

$$
\begin{aligned}
\omega(x)=\frac{\dot{u}(x)}{\ddot{u}\left(\tau_{i}\right)} c(x)=\frac{\varepsilon^{2} \dot{u}(x)}{f_{i}} c(x), \quad c(x)=\frac{\alpha\left(\tau_{i}\right)}{\alpha(x)}\{1+O(\varepsilon)\}, \\
\begin{aligned}
\varepsilon^{2} \dot{u}^{2}(x) \frac{\dot{c}(x)}{c(x)}= & -G_{x}\left(u(x), u_{i}, x\right) \\
& +\int_{\tau_{i}}^{x}\left\{G_{x x}\left(u(t), u_{i}, t\right)-\frac{G_{x}^{2}\left(u(t), u_{i}, t\right)}{\varepsilon^{2} \dot{u}^{2}(t)}\right\} d t+O\left(\varepsilon^{2}\right) \varepsilon^{2} \dot{u}^{2}(x) .
\end{aligned}
\end{aligned}
$$

Proof. For definiteness, we consider the case when $\tau_{i}$ is a local minimum and $x \in$ $\left[\tau_{i}, z_{i+1 / 2}\right]$. We divide the proof into several steps.

Step 1. Write $\omega(x)=\frac{\dot{u}(x)}{\ddot{u}\left(\tau_{i}\right)} c(x)$, differentiate twice and use (27) to obtain $\varepsilon^{2} \dot{u} \ddot{c}+2 \varepsilon^{2} \ddot{u}=-f_{x} c$. Use of L'hôpital's rule shows that $c \in C^{2}$ and $c\left(\tau_{i}\right)=1$. Since $\varepsilon^{2} \ddot{u}=f_{u} \dot{u}+f_{x}$ we find that

$$
\varepsilon^{2} \dot{u}^{2}(x) \dot{c}(x)=-\int_{\tau_{i}}^{x} c(t) \dot{u}(t) f_{x}(u(t), t) d t .
$$

Since $\left|f_{x}\right|=O(u), \int_{\tau_{i}}^{x} \dot{u}\left|f_{x}\right| d t=O(1)\left(u^{2}-u_{i}^{2}\right)=O(1) \varepsilon^{2} \dot{u}^{2}$. Thus, $|\dot{c}(x)| \leq$ $O(1) \max _{t \in\left[\tau_{i}, x\right]}|c(t)|$. A Gronwall's inequality then gives us $\max _{t \in\left[\tau_{i}, z_{i+1 / 2}\right]}\{|c(t)|+$ $|\dot{c}(t)|\}=O(1)$.

Step 2. Write $\dot{u}(t) f_{x}(u(t), t)=\frac{d}{d t} G_{x}\left(u(t), u_{i}, t\right)-G_{x x}\left(u(t), u_{i}, t\right)$. Integration by parts gives

$$
\varepsilon^{2} \dot{u}^{2} \dot{c}=-c G_{x}+\int_{\tau_{i}}^{x}\left\{c G_{x x}+\dot{c} G_{x}\right\} d t,
$$

where $G=G\left(u(t), u_{i}, t\right)$. Divide by $\varepsilon^{2} \dot{u}^{2}$, substitute the result for $\dot{c}$ in the integral, and we get

$$
\begin{aligned}
\varepsilon^{2} \dot{u}^{2} \dot{c} & =-c G_{x}+\int_{\tau_{i}}^{x} c\left\{G_{x x}-\frac{G_{x}^{2}}{\varepsilon^{2} \dot{u}^{2}}\right\} d t+R_{1} \\
& =c\left\{-G_{x}+\int_{\tau_{i}}^{x}\left\{G_{x x}-\frac{G_{x}^{2}}{\varepsilon^{2} \dot{u}^{2}}\right\} d t\right\}+R_{1}+R_{2}
\end{aligned}
$$


where

$$
\begin{aligned}
& R_{1}=\int_{\tau_{i}}^{x} \frac{G_{x}}{\varepsilon^{2} \dot{u}^{2}} \int_{\tau_{i}}^{t}\left[c G_{x x}+\dot{c} G_{x}\right] d \xi d t \\
& R_{2}=-\int_{\tau_{i}}^{x} \dot{c} \int_{\tau_{i}}^{t}\left\{G_{x x}-\frac{G_{x}^{2}}{\varepsilon^{2} \dot{u}^{2}}\right\} d \xi d t .
\end{aligned}
$$

Using the boundedness of $c$ and $\dot{c}$, the fact that

$$
\left|\frac{\partial^{k}}{\partial x^{k}} G\left(u, u_{i}, x\right)\right|=\left|\int_{u_{i}}^{u} \frac{\partial^{k}}{\partial x^{k}} f(s, x) d s\right|=O(1)\left(u^{2}-u_{i}^{2}\right)=O(1) \varepsilon^{2} \dot{u}^{2}
$$

and that $|d x|=\frac{O(\varepsilon) d u}{\sqrt{u^{2}-u_{i}^{2}}}$, we then see that

$$
\begin{aligned}
\left|R_{1}(x)\right| & \leq O\left(\varepsilon^{2}\right) \int_{u_{i}}^{u(x)} \frac{v^{2}-u_{i}^{2}}{\left(v^{2}-u_{i}^{2}\right)^{3 / 2}} d v \int_{u_{i}}^{v} \frac{s^{2}-u_{i}^{2}}{\left(s^{2}-u_{i}^{2}\right)^{1 / 2}} d s \\
& =O\left(\varepsilon^{2}\right)\left(u^{2}-u_{i}^{2}\right)=O\left(\varepsilon^{2}\right) \varepsilon^{2} \dot{u}^{2} .
\end{aligned}
$$

Similarly we have $\left|R_{2}\right|=O\left(\varepsilon^{2}\right) \varepsilon^{2} \dot{u}^{2}$.

Step 3. By the same estimate as for $R_{1}$, the integral in (32) is bounded by $O(\varepsilon) \varepsilon^{2} \dot{u}^{2}$. From the estimates above on $G_{x}$ and $G_{x x}$ we obtain from (33) that

$$
\dot{c}=c\left\{-\frac{G_{x}}{\varepsilon^{2} \dot{u}^{2}}+O(\varepsilon)\right\}+O\left(\varepsilon^{2}\right) .
$$

Note that, for $x$ sufficiently close to $\tau_{i}, u>0$ is sufficiently small, and so

$$
G_{x}=\int_{u_{i}}^{u}\left(f_{x u}(0, x) s+O\left(s^{2}\right)\right) d s=\left\{\frac{1}{2} f_{x u}(0, x)+O(u)\right\}\left(u^{2}-u_{i}^{2}\right)
$$

and

$$
\varepsilon^{2} \dot{u}^{2}=2[1+O(\varepsilon)] G=\left[f_{u}(0, x)+O(\varepsilon)+O(u)\right]\left(u^{2}-u_{i}^{2}\right),
$$

and so

$$
\frac{G_{x}}{\varepsilon^{2} \dot{u}^{2}}=\frac{f_{x u}(0, x)}{2 f_{u}(0, x)}+O(\varepsilon)+O(u) .
$$

Since $\frac{\left|G_{x}\right|}{\varepsilon^{2} \dot{u}^{2}}=O(1)$ and $\frac{\left|f_{x u}(0, x)\right|}{\left|f_{u}(0, x)\right|}=O(1)$ on $\left[\tau_{i}, z_{i+1 / 2}\right]$, we see that this estimate holds on this entire interval. It then follows that

$$
\dot{c}=c\left\{-\frac{f_{x u}(0, x)}{2 f_{u}(0, x)}+O(\varepsilon)+O(u)\right\}+O\left(\varepsilon^{2}\right) .
$$

Solving this "linear" equation we then obtain (31) since $\alpha(x)=\sqrt{f_{u}(0, x)}, c\left(\tau_{i}\right)=$ $1, \int_{\tau_{i}}^{x} u d t=O(\varepsilon)$ and $\exp \left(-\int_{\tau_{i}}^{x} \frac{f_{u x}(0, t)}{2 f_{u}(0, t)} d t\right)=\sqrt{\frac{f_{u}\left(0, \tau_{i}\right)}{f_{u}(0, x)}}$.

Since $\alpha$ has a positive lower bound, it follows from (31) that $c$ also has a positive lower bound. Then dividing (33) by $c$ we get (32). This completes the proof.

\subsection{The function $w_{1}$.}

Lemma 8.2. Let $\omega_{1}$ be the solution to (27). Then the following hold:

(i) If $f_{i}:=f\left(u_{i}, \tau_{i}\right)=o(1)$, then $w_{1}>0$ in $\left[z_{i-1 / 2}, z_{i+1 / 2}\right]$ and

$$
\left.\frac{\varepsilon \omega_{1}}{\omega}\right|_{x=z_{i \pm 1 / 2} \mp 0}= \pm \alpha_{i}+O(\varepsilon)+O\left(f_{i}\right) .
$$


(ii) If $z_{i+1 / 2}-z_{i-1 / 2}=O(\varepsilon)$ and $F_{i}=O(\varepsilon)$, i.e., $\tau_{i}$ is the peak of a spike, then

$$
\left.\frac{\varepsilon \omega_{1}}{\omega}\right|_{x=z_{i \pm 1 / 2} \mp 0}= \pm\left|f_{i}\right|\left\{\frac{1}{\sqrt{2 F\left(\phi_{i}, \tau_{i}\right)}}-\int_{\phi_{i}}^{\phi_{i}^{*}} \frac{f\left(s, \tau_{i}\right)-f\left(\phi_{i}^{*}, \tau_{i}\right)}{\left(2 F\left(s, \tau_{i}\right)\right)^{3 / 2}} d s\right\}+O(\varepsilon) .
$$

Proof. We first derive an integral equation for $\omega_{1}$ on $\left[z_{i-1 / 2}, z_{i+1 / 2}\right]$. Since $\dot{\omega}_{1} \omega-$ $\dot{\omega} \omega_{1}=-1, \dot{\omega}\left(\tau_{i}\right)=1$ and $\omega(x) \neq 0$ for $x \neq \tau_{i}$, we have $\frac{d}{d x}\left(\omega_{1} / \omega\right)=-1 / \omega^{2}$ for $x \neq \tau_{i}$, and so

$$
\frac{d}{d x} \frac{\omega_{1}-1}{\omega}=\frac{d}{d x}\left(\frac{\omega_{1}}{\omega}\right)-\frac{d}{d x}\left(\frac{1}{\omega}\right)=\frac{\dot{\omega}-1}{\omega^{2}}=\frac{\dot{\omega}-\dot{\omega}\left(\tau_{i}\right)}{\omega^{2}} .
$$

Hence, for $\tau, x \in\left[z_{i-1 / 2}, \tau_{i}\right)$ or $\tau, x \in\left(\tau_{i}, z_{i+1 / 2}\right]$,

$$
\frac{\omega_{1}(x)-1}{\omega(x)}=\frac{\omega_{1}(\tau)-1}{\omega(\tau)}+\int_{\tau}^{x} \frac{\dot{\omega}(t)-\dot{\omega}\left(\tau_{i}\right)}{\omega^{2}(t)} d t
$$

Note that

$\lim _{\tau \rightarrow \tau_{i}} \frac{\omega_{1}(\tau)-1}{\omega(\tau)}=\lim _{\tau \rightarrow \tau_{i}} \frac{\dot{\omega}_{1}(\tau)}{\dot{\omega}(\tau)}=0, \quad \lim _{\tau \rightarrow \tau_{i}} \frac{\dot{\omega}(\tau)-1}{\omega^{2}(\tau)}=\lim _{\tau \rightarrow \tau_{i}} \frac{\ddot{\omega}(\tau)}{2 \omega(\tau) \dot{\omega}(\tau)}=\frac{f_{u}\left(u_{i}, \tau_{i}\right)}{2 \varepsilon^{2}}$.

Then, sending $\tau \rightarrow \tau_{i}$ in the above equation leads to, for $x \in\left[z_{i-1 / 2}, z_{i+1 / 2}\right]$,

$$
\omega_{1}(x)=1+\omega(x) \int_{\tau_{i}}^{x} \frac{\dot{\omega}(t)-\dot{\omega}\left(\tau_{i}\right)}{\omega^{2}(t)} d t .
$$

For definiteness, we consider the case when $\tau_{i}$ is a local minimum and $x \in$ $\left(\tau_{i}, z_{i+1 / 2}\right)$. Then $\dot{\omega}(t)-\dot{\omega}\left(\tau_{i}\right)=\int_{\tau_{i}}^{t} \ddot{\omega}(\eta) d \eta=\varepsilon^{-2} \int_{\tau_{i}}^{t} f_{u} \omega d \eta$. Using $\omega=\frac{\varepsilon^{2} \dot{u}}{f_{i}} c$, $c(\eta)=1+O\left(\varepsilon+\eta-\tau_{i}\right)$, and $f_{u}(u, \eta)=f_{u}\left(u, \tau_{i}\right)+O\left(\eta-\tau_{i}\right)$, we then have

$$
\begin{aligned}
\dot{\omega}(t)-\dot{\omega}\left(\tau_{i}\right) & =\frac{1}{f_{i}} \int_{\tau_{i}}^{t}\left\{\dot{u} f_{u}\left(u, \tau_{i}\right)+\dot{u} O\left(\varepsilon+\eta-\tau_{i}\right)\right\} d \eta \\
& =\frac{1}{f_{i}}\left\{f\left(u, \tau_{i}\right)-f_{i}+O\left(\varepsilon+t-\tau_{i}\right)\left(u-u_{i}\right)\right\} \\
\int_{\tau_{i}}^{x} \frac{\dot{\omega}-\dot{\omega}\left(\tau_{i}\right)}{\omega^{2}} d t & =\frac{\mathrm{H}(\dot{u}) f_{i}}{\varepsilon} \int_{u_{i}}^{u(x)} \frac{f\left(u, \tau_{i}\right)-f_{i}+O\left(\varepsilon+t-\tau_{i}\right)\left(u-u_{i}\right)}{[1+O(\varepsilon)]\left[2 G\left(u, u_{i}, t\right)\right]^{3 / 2}} d u(t) .
\end{aligned}
$$

Case (i): $f_{i}=o(1)$. We use $G\left(u, u_{i}, t\right)^{-1}=G\left(u, u_{i}, \tau_{i}\right)^{-1}\left[1+O\left(\left|t-\tau_{i}\right|\right)\right]$ and

$$
\begin{aligned}
{\left[2 G\left(u, u_{i}, \tau_{i}\right)\right]^{-3 / 2} } & =\left\{f_{u}\left(0, \tau_{i}\right)\left(u^{2}-u_{i}^{2}\right)\right\}^{-3 / 2}\left\{1-\frac{f_{u u}\left(0, \tau_{i}\right)}{2 f_{u}\left(0, \tau_{i}\right)} \frac{u^{2}+u u_{i}+u_{i}^{2}}{u+u_{i}}+O\left(u^{2}\right)\right\}, \\
f\left(u, \tau_{i}\right)-f_{i} & =f_{u}\left(0, \tau_{i}\right)\left(u-u_{i}\right)\left\{1+\frac{f_{u u}\left(0, \tau_{i}\right)}{2 f_{u}\left(0, \tau_{i}\right)}\left(u+u_{i}\right)+O\left(u^{2}\right)\right\} .
\end{aligned}
$$

Also using $\left|t-\tau_{i}\right|=O(\varepsilon) \ln \left(u / u_{i}\right)$ we obtain

$$
\begin{aligned}
\int_{\tau_{i}}^{x} \frac{\dot{\omega}-\dot{\omega}\left(\tau_{i}\right)}{\omega^{2}} d t & =\frac{\mathrm{H}(\dot{u}) f_{i}}{\varepsilon} \int_{u_{i}}^{u(x)} \frac{1+\frac{f_{u u}\left(0, \tau_{i}\right)}{2 f_{u}\left(0, \tau_{i}\right.} \frac{u u_{i}}{u+u_{i}}+O\left(u^{2}\right)+O(\varepsilon) \ln u / u_{i}}{\sqrt{f_{u}\left(0, \tau_{i}\right)}\left(u+u_{i}\right)\left(u^{2}-u_{i}^{2}\right)^{1 / 2}} d u \\
& =\frac{\mathrm{H}(\dot{u}) f_{i}}{\varepsilon \alpha_{i} u_{i}} \int_{1}^{u / u_{i}} \frac{1+O(1) \frac{s}{1+s} u_{i}+O(1) s^{2} u_{i}^{2}+O(\varepsilon) \ln s}{(1+s) \sqrt{s^{2}-1}} d s \\
& =\frac{\mathrm{H}(\dot{u}) f_{i}}{\varepsilon \alpha_{i} u_{i}}\left\{\int_{1}^{u / u_{i}} \frac{d s}{(1+s) \sqrt{s^{2}-1}} d s+O(\varepsilon)+O\left(u_{i}\right)\right\} .
\end{aligned}
$$


Since $\int_{1}^{\infty} \frac{1}{(1+s) \sqrt{s^{2}-1}} d s=1$, we then obtain

$\left.\frac{\varepsilon \omega_{1}}{\omega}\right|_{x=z_{i \pm 1 / 2}}=\frac{[1+O(\varepsilon)] f_{i}}{\varepsilon \dot{u}}+\frac{\mathrm{H}(\dot{u}) f_{i}}{\alpha_{i} u_{i}}\left\{1+O\left(f_{i}\right)+O(\varepsilon)\right\}= \pm \alpha_{i}+O\left(f_{i}\right)+O(\varepsilon)$

since $f_{i}=\alpha_{i}^{2} u_{i}+O\left(u_{i}^{2}\right)$. The assertion (34) thus follows.

Case (ii): $F_{i}=O(\varepsilon)$ and $z_{i+1 / 2}-z_{i-1 / 2}=O(\varepsilon)$. Then since $G\left(\cdot, u_{i}, t\right)$ vanishes linearly at $u_{i}$,

$$
\frac{\varepsilon \omega_{1}}{\omega}=\frac{[1+O(\varepsilon)] f_{i}}{\varepsilon \dot{u}}+\mathrm{H}(\dot{u}) f_{i} \int_{u_{i}}^{u(x)} \frac{f\left(u, \tau_{i}\right)-f_{i}}{\left[2 G\left(u, u_{i}, t\right)\right]^{3 / 2}} d u(t)+O(\varepsilon) .
$$

Using $G\left(s, u_{i}, t\right)=[1+O(\varepsilon)] G\left(s, u_{i}, \tau_{i}\right), u_{i}-\phi_{i}^{*}=O(\varepsilon)$, the change of variables $\hat{s}=s+\phi_{i}^{*}-u_{i}$ and the fact that $G\left(s, u_{i}, \tau_{i}\right)=[1+O(\varepsilon)] G\left(\hat{s}, \phi_{i}^{*}, \tau_{i}\right) \propto \hat{s}-\phi_{i}^{*}$ and $f\left(s, \tau_{i}\right)-f\left(u_{i}, \tau_{i}\right)=f\left(\hat{s}, \tau_{i}\right)-f\left(\phi_{i}^{*}, \tau_{i}\right)+O(\varepsilon)\left(\hat{s}-\phi_{i}^{*}\right)$, we then have

$$
\int_{u_{i}}^{u(x)} \frac{f\left(u, \tau_{i}\right)-f_{i}}{\left[2 G\left(u, u_{i}, t\right)\right]^{3 / 2}} d u(t)=\int_{\phi_{i}^{*}}^{u(x)+\phi_{i}^{*}-u_{i}} \frac{f\left(\hat{s}, \tau_{i}\right)-f\left(\phi_{i}^{*}, \tau_{i}\right)}{\left[2 G\left(\hat{s}, \phi_{i}^{*}, \tau_{i}\right)\right]^{3 / 2}} d \hat{s}+O(\varepsilon) .
$$

Finally, noting that $F\left(s, \tau_{i}\right)=G\left(s, \phi_{i}^{*}, \tau_{i}\right)$ and $\varepsilon \dot{u}=\mathrm{H}(\dot{u})[1+O(\varepsilon)] \sqrt{2 G\left(u, u_{i}, x\right)}=$ $\mathrm{H}(\dot{u})[1+O(\varepsilon)] \sqrt{2 F\left(u, \tau_{i}\right)}$ if $x=z_{i \pm 1 / 2}$, and also $\mathrm{H}\left(\dot{u}_{i+1 / 2}\right)=\mathrm{H}\left(f_{i}\right)$, we then obtain the assertion (35).

The case when $u\left(\tau_{i}\right)$ is a local maximum can also be similarly treated.

\subsection{Basic estimates on the number of roots of $w=0$.}

Lemma 8.3. Let $\psi$ be a solution to $\varepsilon^{2} \ddot{\psi}=f_{u} \psi$.

(i) For each $i$, there exist at most two roots of $\psi=0$ in $\left[\tau_{i}, \tau_{i+1}\right]$.

(ii) If $f_{i}=o(1)$ and $\psi\left(\tau_{i}\right) \dot{\psi}\left(\tau_{i}\right) \geq 0$, then $\psi=0$ has at most one root in $\left(\tau_{i}, \tau_{i+1}\right]$.

(iii) If $f_{i}^{2} \propto 1$, then $\psi=0$ has at least one root in $\left(\tau_{i-1}, \tau_{i+1}\right)$, and at most three roots in $\left[\tau_{i-1}, \tau_{i+1}\right]$.

Proof. (i) Note that $\omega \neq 0$ in $\left(\tau_{i}, z_{i+1 / 2}-0\right]$ and $\left[z_{i+1 / 2}+0, \tau_{i+1}\right)$. Hence, by the Liouville theorem, $\psi=0$ can have at most two roots in $\left[\tau_{i}, \tau_{i+1}\right]$ for all $i$.

(ii) When $f_{i}=o(1)$, we have, for all $x \in\left(\tau_{i}, z_{i+1 / 2}\right], \omega(x)>0, \omega_{1}(x)>0$ and $\psi(x)=\psi\left(\tau_{i}\right) \omega_{1}(x)+\dot{\psi}\left(\tau_{i}\right) \omega(x) \neq 0$. The proof of (i) shows that $\psi=0$ can have at most one root in $\left(z_{i+1 / 2}, \tau_{i+1}\right]$. Thus, $\psi=0$ has at most one root in $\left(\tau_{i}, \tau_{i+1}\right]$.

(iii) First consider the case when $\psi\left(\tau_{i-1}\right)=0$ and $\dot{\psi}\left(\tau_{i-1}\right)=\varepsilon \ddot{u}\left(\tau_{i-1}\right)$. Then $\frac{\psi}{\varepsilon \dot{u}}=c=(1+O(\varepsilon)) \frac{\alpha\left(\tau_{i-1}\right)}{\alpha(x)}$ and $\varepsilon \dot{\psi}=O(\varepsilon)$ at $z_{i-1 / 2}$. As the length of the interval $\left[z_{i-1 / 2}, z_{i+1 / 2}\right]$ is of order $\varepsilon$, we use a stretched variable $y=\left(x-\tau_{i}\right) / \varepsilon$ and set $V(y)=\frac{\varepsilon \dot{u}_{i-1 / 2}}{\psi\left(z_{i-1 / 2}\right)} \psi(x)$ and $V_{1}(y)=\varepsilon \dot{u}(x)$. We find that $V^{\prime \prime}(y)=f_{u}(u(x), x) V(y)$ and $V_{1}^{\prime \prime}(y)=f_{u}(u(x), x) V_{1}(y)+O(\varepsilon)$. Then, a regular perturbation argument shows that $V(y)=V_{1}(y)+O(\varepsilon)$ for $y \in\left[\left(z_{i-1 / 2}-\tau_{i}\right) / \varepsilon,\left(z_{i+1 / 2}-\tau_{i}\right) / \varepsilon\right]$, and so $\psi=\frac{\psi\left(z_{i-1 / 2}\right)}{\varepsilon \dot{u}_{i-1 / 2}}[\varepsilon \dot{u}+O(\varepsilon)]$ in $\left[z_{i-1 / 2}, z_{i+1 / 2}\right]$. This implies that in the interval $\left(\tau_{i-1}, z_{i+1 / 2}\right], \psi=0$ has exactly one root at $\xi=\tau_{i}+O\left(\varepsilon^{2}\right)$. As $\psi=0$ can have at most one root in $\left[z_{i+1 / 2}, \tau_{i+1}\right]$, we see that $\psi=0$ has at most two roots in $\left(\tau_{i-1}, \tau_{i+1}\right]$.

Now suppose that $\psi$ is a generic solution. Then, by the Liouville theorem, $\psi=0$ can have at most three roots in $\left[\tau_{i-1}, \tau_{i+1}\right]$, and there is exactly one root in $\left(\tau_{i-1}, \xi\right]$. This completes the proof. 


\section{The Morse indeX For LAYERED SOLUtions}

We first consider a cluster of layers. For convenience, if $\left(\tau_{-k}, \tau_{k}\right)$ (or $\left.\left(\tau_{l}, \tau_{2 n-l}\right)\right)$ is an interval with a boundary cluster, we say that $\left(\tau_{0}, \tau_{k}\right)\left(\right.$ or $\left.\left(\tau_{l}, \tau_{n}\right)\right)$ is an interval with a boundary cluster.

Theorem 6. Assume that $\left(\tau_{l}, \tau_{k}\right) \subset(a, b)$ is an interval with a cluster of layers. Let $w$ be the solution to (26) and assume that $w\left(\tau_{l}\right) \dot{w}\left(\tau_{l}\right) \geq 0$. Then, $w\left(\tau_{k}\right) \dot{w}\left(\tau_{k}\right)>0$ except for $k=n$. Also, the following hold:

(i) If $\left(\tau_{l}, \tau_{k}\right)$ is a boundary cluster at a, or an interior cluster with multiple layers, or an interior cluster with a single layer with $\dot{u}_{l+1 / 2} \dot{J}\left(z_{l+1 / 2}\right)>0$, then $w=0$ has exactly $k-l$ roots in $\left(\tau_{l}, \tau_{k}\right)$ and

$$
\frac{\varepsilon \dot{w}\left(\tau_{k}\right)}{w\left(\tau_{k}\right)}=\alpha_{k}+o(1)
$$

(ii) If $\left(\tau_{l}, \tau_{k}\right)$ with $k=l+1$ is an interval with an interior cluster of a single layer with $\dot{J}\left(z_{l+1 / 2}\right) \dot{u}_{l+1 / 2}<0$, then $w=0$ has no root in $\left(\tau_{l}, \tau_{l+1}\right)$ and (36) holds.

(iii) If $\left(\tau_{l}, \tau_{k}\right)=\left(\tau_{l}, b\right)$ is a boundary cluster at $b$, then one of the following alternative holds:

either $w=0$ has $n-l$ roots in $\left(\tau_{l}, b\right)$ and $w(b) \dot{w}(b)>0$

or $w=0$ has $n-l-1$ roots in $\left(\tau_{l}, b\right)$ and $w(b) \dot{w}(b) \leq 0, \dot{w}(b) \neq 0$.

9.1. The matrix $\mathbf{P} \Psi\left(\tau_{i+1}, \tau_{i}\right) \mathbf{P}^{-1}$. Assume that for $j=i$ and $i+1, f_{j}^{2}=\mu_{j} \varepsilon|\ln \varepsilon|$, where either $\mu_{j} \propto 1$ or $\mu_{j}=O\left(e^{-1 / \sqrt{\varepsilon}}\right)$. We find an asymptotic expansion for $\mathbf{P} \Psi\left(\tau_{i+1}, \tau_{i}\right) \mathbf{P}^{-1}$ given by (29).

First of all, by (34) and the definition of $\mathbf{t}$ and $\mathbf{t}^{\perp}$ in (28),

$$
\mathbf{t}_{+}^{\perp}=\left[\begin{array}{c}
1 \\
\alpha_{i+1}
\end{array}\right]+O(\sqrt{\varepsilon|\ln \varepsilon|}), \quad \mathbf{t}_{-}=\left[\begin{array}{c}
\alpha_{i} \\
1
\end{array}\right]+O(\sqrt{\varepsilon|\ln \varepsilon|}) .
$$

Note that for $t_{+}^{\perp}$, we need $\frac{\varepsilon \omega_{1}}{\omega}$ at $z_{(i+1)-\frac{1}{2}}+0$, so that the minus sign is chosen on the right side of (34).

Next, at $x=z_{i+1 / 2}, \ddot{u}=0$ so that $\rho=\frac{\dot{\omega}}{\omega}=\frac{\dot{c}}{c}$. Thus by (32) where the integral is $O(\varepsilon)$, and (31),

$$
\begin{aligned}
\varepsilon^{2} \dot{u}_{i+1 / 2}^{2} \llbracket \rho \rrbracket_{-}^{+} & =G_{x}\left(\phi_{i+1 / 2}, u_{i}, z_{i+1 / 2}\right)-G_{x}\left(\phi_{i+1 / 2}, u_{i+1}, z_{i+1 / 2}\right)+O(\varepsilon) \\
& =\int_{u_{i}}^{u_{i+1}} f_{x}\left(s, z_{i+1 / 2}\right) d s+O(\varepsilon) \\
& =\mathrm{H}\left(\dot{u}_{i+1 / 2}\right) \dot{J}\left(z_{i+1 / 2}\right)+O\left(u_{i}^{2}\right)+O\left(u_{i-1}^{2}\right)+O(\varepsilon) \\
& =\mathrm{H}\left(\dot{u}_{i+1 / 2}\right) \dot{J}\left(z_{i+1 / 2}\right)+O(\varepsilon|\ln \varepsilon|), \\
\frac{\varepsilon^{3} \dot{u}_{i+1 / 2}^{2}}{\omega_{-}^{2}} & =\frac{\varepsilon^{3} \dot{u}_{i+1 / 2}^{2} f_{i}^{2}}{\varepsilon^{4} \dot{u}_{i+1 / 2}^{2} c^{2}\left(z_{i+1 / 2}-0\right)}=\mu_{i}|\ln \varepsilon|+O\left(\varepsilon|\ln \varepsilon|^{2}\right), \\
\frac{\varepsilon^{3} \dot{u}_{i+1 / 2}^{2}}{\omega_{+}^{2}} & =\frac{\varepsilon^{3} \dot{u}_{i+1 / 2}^{2} f_{i}^{2}}{\varepsilon^{4} \dot{u}_{i+1 / 2}^{2} c^{2}\left(z_{i+1 / 2}+0\right)}=\mu_{i+1}|\ln \varepsilon|+O\left(\varepsilon|\ln \varepsilon|^{2}\right),
\end{aligned}
$$

where we have used the fact that $c\left(z_{i+1 / 2}\right)=1+O\left(\tau_{j}-z_{i+1 / 2}\right)(j=i$ or $i+1)$ and either $\left|\tau_{j}-z_{i+1 / 2}\right|=O(\varepsilon|\ln \varepsilon|)$ or $f_{j}=O\left(e^{-1 / \sqrt{\varepsilon}}\right)$. 
Hence, it follows from (29) that

$$
\begin{aligned}
\mathbf{P} \Psi\left(\tau_{i+1}, \tau_{i}\right) \mathbf{P}^{-1}=- & r\left\{\left[\begin{array}{c}
1 \\
\alpha_{i+1}
\end{array}\right]\left[\begin{array}{ll}
\mu_{i} & 0
\end{array}\right]+\left[\begin{array}{c}
0 \\
\mu_{i+1}
\end{array}\right]\left[\begin{array}{ll}
\alpha_{i} & 1
\end{array}\right]\right. \\
& \left.+\frac{\mathrm{H}\left(\dot{u}_{i+1 / 2}\right) \dot{J}\left(z_{i+1 / 2}\right)}{|\ln \varepsilon|}\left[\begin{array}{c}
1 \\
\alpha_{i+1}
\end{array}\right]\left[\begin{array}{ll}
\alpha_{i} & 1
\end{array}\right]+O(\sqrt{\varepsilon|\ln \varepsilon|})\right\},
\end{aligned}
$$

where

$$
r=-\frac{\omega_{-} \omega_{+}|\ln \varepsilon|}{\varepsilon^{3} \dot{u}_{i+1 / 2}^{2}} .
$$

Since $\omega_{-} \omega_{+}=\frac{\varepsilon^{4} \dot{u}_{i+1 / 2}^{2}}{f_{i} f_{i+1}} c\left(z_{i+1 / 2}-0\right) c\left(z_{i+1 / 2}+0\right)<0$, we have $r>0$.

9.2. Boundary layers at $a$. Suppose that $\left(\tau_{0}, \tau_{k}\right)$ is an interval with a cluster of boundary layers. Then $\mu_{i} \propto 1$ for $i=0, \cdots, k-1$ and $\mu_{k}=O\left(e^{-1 / \sqrt{\varepsilon}}\right)$. Using $w(a)=1, \dot{w}(a)=0$ and (38), we inductively derive that

$$
\tilde{\mathbf{w}}\left(\tau_{i}\right)=(-1)^{i}\left|w\left(\tau_{i}\right)\right|\left[\begin{array}{c}
1 \\
\nu_{i}+o(1)
\end{array}\right]
$$

for $i=0, \cdots, k$, where $\nu_{i}$ is inductively calculated by

$$
\nu_{0}=0, \quad \nu_{i+1}=\alpha_{i+1}+\frac{\mu_{i+1}}{\mu_{i}}\left(\alpha_{i}+\nu_{i}\right)>0 \quad \forall i=0, \cdots, k-1 .
$$

In particular, $\nu_{k}=\alpha_{k}+O\left(e^{-\sqrt{\varepsilon}}\right)$. Therefore, we conclude that:

(i) $(-1)^{i} w\left(\tau_{i}\right)>0,(-1)^{i} \dot{w}\left(\tau_{i}\right)>0$ for all $i=1, \cdots, k$,

(ii) $w=0$ has exactly one root in each $\left(\tau_{i}, \tau_{i+1}\right)$ for $i=0, \cdots, k-1$, and

(iii) $w=0$ has $k$ roots in $\left(a, \tau_{k}\right]$ and (36) holds.

9.3. Interior single layer. Next we consider the case when $\left(\tau_{l}, \tau_{k}\right)$ with $k=l+1$ is an interval with an interior cluster of a single layer. Then $\mu_{l}, \mu_{l+1}=O\left(e^{-1 / \sqrt{\varepsilon}}\right)$. Using (38) and $w\left(\tau_{l}\right) \dot{w}\left(\tau_{l}\right) \geq 0$ (so that $\mathrm{H}\left(\alpha_{l} w_{l}+\varepsilon \dot{w}_{l}\right)=\mathrm{H}\left(w_{l}+\dot{w}_{l}\right)$ ) we obtain

$$
\begin{aligned}
\tilde{\mathbf{w}}\left(\tau_{l+1}\right) & =\mathbf{P} \Psi\left(\tau_{l+1}, \tau_{l}\right) \mathbf{P}^{-1} \tilde{\mathbf{w}}\left(\tau_{l}\right) \\
& =-r \frac{\mathrm{H}\left(\dot{u}_{l+1 / 2}\right) \dot{J}\left(z_{l+1 / 2}\right)}{|\ln \varepsilon|}\left(\alpha_{l} w_{l}+\varepsilon \dot{w}_{l}\right)(1+o(1))\left[\begin{array}{c}
1 \\
\alpha_{l+1}+o(1)
\end{array}\right] \\
& =(-1)^{s}\left|w\left(\tau_{l+1}\right)\right|\left[\begin{array}{c}
1 \\
\alpha_{l+1}+o(1)
\end{array}\right] \mathrm{H}\left(w_{l}+\dot{w}_{l}\right),
\end{aligned}
$$

where $s=0$ if $\dot{u}_{l+1 / 2} \dot{J}\left(z_{l+1 / 2}\right)<0$ and $s=1$ if $\dot{u}_{l+1 / 2} \dot{J}\left(z_{l+1 / 2}\right)>0$. Hence, (36) holds, and $w=0$ has no root in $\left(\tau_{l}, \tau_{l+1}\right]$ if $\dot{u}_{l+1 / 2} \dot{J}\left(z_{l+1 / 2}\right)<0$, and $w=0$ has exactly one root in $\left(\tau_{l}, \tau_{l+1}\right)$ if $\dot{u}_{l+1 / 2} \dot{J}\left(z_{l+1 / 2}\right)>0$.

9.4. Interior multiple layers. Now we consider the case that $\left(\tau_{l}, \tau_{k}\right)$ is an interval with an interior cluster of multiple layers. Then $k-l$ is odd and $>1$, $\dot{u}_{l+1 / 2} \dot{J}\left(z_{l+1 / 2}\right)>0, \mu_{l}=O\left(e^{-1 / \sqrt{\varepsilon}}\right), \mu_{k}=O\left(e^{-1 / \sqrt{\varepsilon}}\right)$, and $\mu_{i} \propto 1$ for $i=$ $l+1, \cdots, k-1$. For simplicity, we assume that $w\left(\tau_{l}\right) \geq 0$ and $\dot{w}\left(\tau_{l}\right) \geq 0$. Using (38) and a mathematical induction we derive that, for $i=l+1, \cdots, k$,

$$
\tilde{\mathbf{w}}\left(\tau_{i}\right)=(-1)^{i-l}\left|\varepsilon \dot{w}\left(\tau_{i}\right)\right|\left[\begin{array}{c}
a_{i} \\
1
\end{array}\right]
$$


where, for $m=1,2, \cdots, \frac{k-l-1}{2}$,

$$
\begin{aligned}
a_{l+2 m-1} & =\frac{\left|\dot{J}\left(z_{l+1 / 2}\right)\right|}{\mu_{l+2 m-1}|\ln \varepsilon|}+O\left(\frac{1}{|\ln \varepsilon|^{2}}\right), \\
a_{l+2 m} & =-\frac{2\left|\dot{J}\left(z_{l+1 / 2}\right)\right|^{2}}{\mu_{l+2 m}|\ln \varepsilon|^{2}} \sum_{s=1}^{m} \frac{\alpha_{l+2 s-1}}{\mu_{l+2 s-1}}+O\left(\frac{1}{|\ln \varepsilon|^{3}}\right),
\end{aligned}
$$

while $a_{k}=\frac{1}{\alpha_{k}}+O\left(\frac{1}{|\ln \varepsilon|}\right)$.

In the first step we verify (39) for $i=l+1$. For convenience, let $d_{i+1 / 2}=$ $\mathrm{H}\left(\dot{u}_{i+1 / 2}\right) \dot{J}\left(z_{i+1 / 2}\right)$. Because $\mu_{l}=O\left(e^{-1 / \sqrt{\varepsilon}}\right)$, (38) gives

$$
\tilde{\mathbf{w}}_{l+1}=\left[\begin{array}{c}
w_{l+1} \\
\varepsilon \dot{w}_{l+1}
\end{array}\right]=-r\left[\begin{array}{c}
\left(\frac{d_{l+1 / 2}}{|\ln \varepsilon|}+O(\sqrt{\varepsilon|\ln \varepsilon|})\right)\left(\alpha_{l} w_{l}+\varepsilon \dot{w}_{l}\right) \\
\left(\mu_{l+1}+\frac{d_{l+1 / 2}}{|\ln \varepsilon|} \alpha_{l+1}+O(\sqrt{\varepsilon|\ln \varepsilon|})\right)\left(\alpha_{l} w_{l}+\varepsilon \dot{w}_{l}\right)
\end{array}\right],
$$

and so

$$
\begin{aligned}
\frac{w_{l+1}}{\varepsilon \dot{w}_{l+1}} & =\frac{\frac{d_{l+1 / 2}}{|\ln \varepsilon|}+O(\sqrt{\varepsilon|\ln \varepsilon|})}{\mu_{l+1}+\alpha_{l+1} \frac{d_{l+1 / 2}}{|\ln \varepsilon|}+O(\sqrt{\varepsilon|\ln \varepsilon|})} \\
& =\frac{d_{l+1 / 2}}{\mu_{l+1}|\ln \varepsilon|}\left\{1-\frac{d_{l+1 / 2}}{|\ln \varepsilon|} \frac{\alpha_{l+1}}{\mu_{l+1}}+O\left(\frac{1}{|\ln \varepsilon|^{2}}\right)\right\},
\end{aligned}
$$

which together with $d_{l+1 / 2}=\left|\dot{J}\left(z_{l+1 / 2}\right)\right|$ in particular proves (39) for $i=l+1$.

For the general induction step we must include the terms involving both $\mu_{i}$ and $\mu_{i+1}$ in the computation. We make the induction hypothesis that (39) holds for $\tilde{\mathbf{w}}\left(\tau_{i}\right)$ so that $w_{i}=a_{i} \varepsilon \dot{w}_{i} \neq 0$. From (38) we obtain

$$
\begin{aligned}
& \frac{w_{i+1}}{\varepsilon \dot{w}_{i+1}} \\
& =\frac{\left(\mu_{i}+\frac{d_{i+1 / 2}}{|\ln \varepsilon|} \alpha_{i}+O(\sqrt{\varepsilon|\ln \varepsilon|})\right) a_{i}+\frac{d_{i+1 / 2}}{|\ln \varepsilon|}+O(\sqrt{\varepsilon|\ln \varepsilon|})}{\left(\alpha_{i+1} \mu_{i}+\mu_{i+1} \alpha_{i}+\frac{d_{i+1 / 2}}{|\ln \varepsilon|} \alpha_{i} \alpha_{i+1}+O(\sqrt{\varepsilon|\ln \varepsilon|})\right) a_{i}+\left(\mu_{i+1}+\frac{d_{i+1 / 2}}{|\ln \varepsilon|} \alpha_{i+1}+O(\sqrt{\varepsilon|\ln \varepsilon|})\right)} .
\end{aligned}
$$

But from the induction hypothesis, $a_{i}=O\left(\frac{1}{|\ln \varepsilon|}\right)$ if $i-l$ is odd and $a_{i}=O\left(\frac{1}{|\ln \varepsilon|^{2}}\right)$ if $i-l$ is even. This allows us to simplify the ratio $\frac{w_{i+1}}{\varepsilon \dot{w}_{i+1}}$. For example, consider the step when $i=l+1$. In this case we obtain

$$
\frac{w_{l+2}}{\varepsilon \dot{w}_{l+2}}=\frac{\mu_{l+1} a_{l+1}+\frac{d_{l+3 / 2}}{|\ln \varepsilon|}+\frac{d_{l+3 / 2}}{|\ln \varepsilon|} \alpha_{l+1} a_{l+1}+O(\sqrt{\varepsilon|\ln \varepsilon|})}{\mu_{l+2}+O\left(\frac{1}{|\ln \varepsilon|}\right)} .
$$

Substituting from (41) gives cancellation of the terms in the numerator of order $\frac{1}{\ln \varepsilon}$ and leads to the desired expression for $a_{2}$. We omit the remaining steps of this induction. We remark that in order to get $a_{l+2 m}$, we need the term of order $\frac{1}{|\ln \varepsilon|^{2}}$ in $a_{l+2 m-1}$ though, for simplicity, we do not give it explicitly in (40).

Now using $\mu_{k}=O\left(e^{-1 / \sqrt{\varepsilon}}\right)$ and the oddness of $k-l$, and (38) for the last layer in the cluster, we get (39) for $i=k$ with $a_{k}=\frac{1}{\alpha_{k}}+O\left(\frac{1}{|\ln \varepsilon|}\right)$, which implies (36).

We now show that $w=0$ has exactly $k-l$ roots in $\left(\tau_{l}, \tau_{k}\right)$. 
First, we see from the expression of $a_{i}$ and $w\left(\tau_{i}\right)$ that $w\left(\tau_{i}\right)<0$ and $(-1)^{i-l} \dot{w}\left(\tau_{i}\right)$ $>0$ for all $i=l, \cdots, k$. Consequently, there is exactly one root of $w=0$ in $\left(\tau_{l}, \tau_{l+1}\right)$ and the number of roots of $w=0$ in $\left(\tau_{i}, \tau_{i+1}\right)$ is even for all $i=l+1, \cdots, k-1$.

When $i-l \in[1, k-l]$ is odd, we have $w\left(\tau_{i}\right) \dot{w}\left(\tau_{i}\right)>0$, so that $w=0$ has at most one root in $\left[\tau_{i}, \tau_{i+1}\right]$, and consequently, there are no roots in $\left[\tau_{i}, \tau_{i+1}\right]$.

When $i-l \in[2, k-l-1]$ is even, we can use

$$
w\left(z_{i+1 / 2}\right)=\dot{w}\left(\tau_{i}\right) \omega\left(z_{i+1 / 2}-0\right)\left\{1+\left.\frac{w\left(\tau_{i}\right)}{\varepsilon \dot{w}\left(\tau_{i}\right)} \frac{\varepsilon \omega_{1}}{\omega}\right|_{x=z_{i+1 / 2}-0}\right\},
$$

along with the fact that $0>\frac{w\left(\tau_{i}\right)}{\varepsilon \dot{w}\left(\tau_{i}\right)}=a_{i}=O\left(\frac{1}{|\ln \varepsilon|^{2}}\right),\left.\frac{\varepsilon \omega_{1}}{\omega}\right|_{x=z_{i+1 / 2}-0}=O(1)$, and $\omega\left(z_{i+1 / 2}-0\right)>0$ to conclude $w\left(z_{i+1 / 2}\right)>0$ so that $w=0$ has exactly one root in $\left(\tau_{i}, z_{i+1 / 2}\right)$. Hence, $w=0$ has two roots in $\left(\tau_{i}, \tau_{i+1}\right)$.

In conclusion, $w=0$ has $k-l$ roots in $\left(\tau_{l}, \tau_{k}\right]$, and (36) holds.

9.5. Boundary layers at $b$. Finally we consider the case when $\left(\tau_{l}, \tau_{n}\right)$ is an interval with a boundary cluster of layers. Then $\mu_{l}=O\left(e^{-1 / \sqrt{\varepsilon}}\right)$ and $\mu_{i} \propto 1$ for $i=l+1, \cdots, n$. Following the same calculation as for multiple interior layers, we have (39) (if $w\left(\tau_{l}\right) \geq 0$ and $\dot{w}\left(\tau_{l}\right) \geq 0$ ) for all $i=l+1, \cdots, k$ with $a_{i}$ given by (40)). Hence, we have the following conclusion:

(1) Suppose that $n-l$ is odd. Then $w=0$ has exactly $n-l$ roots in $\left(\tau_{l}, b\right)$ and $w(b) \dot{w}(b)>0$.

(2) Suppose that $n-l$ is even. Then $w=0$ has exactly $n-l-1 \operatorname{roots}$ in $\left(\tau_{l}, b\right)$ and $w(b) \dot{w}(b)<0$.

\section{The Morse Index For SPIKeS}

In this section, we prove the following:

Theorem 7. Let $\left(\tau_{l}, \tau_{k}\right)$ be an interval with a cluster of spikes. Let $w$ be the solution to (26), and assume that $w\left(\tau_{l}\right) \dot{w}\left(\tau_{l}\right) \geq 0$. Then $w\left(\tau_{k}\right) \dot{w}\left(\tau_{k}\right)>0$ with the only possible exception $k=n$. Also the following hold:

(i) If $\left(\tau_{l}, \tau_{k}\right)$ is a boundary cluster at a, an interior cluster of multiple spikes, or an interior cluster of single spike with $J\left(\tau_{l+1}\right) \dot{S}\left(\tau_{l+1}\right)>0$, then $w=0$ has $k-l$ roots in $\left(\tau_{l}, \tau_{k}\right)$ and (36) holds.

(ii) If $\left(\tau_{l}, \tau_{k}\right)$ with $k=l+2$ is an interior cluster of a single spike with

$$
J\left(\tau_{l+1}\right) \dot{S}\left(\tau_{l+1}\right)<0,
$$

then $w=0$ has exactly one root in $\left(\tau_{l}, \tau_{l+2}\right]$, and (36) holds.

(iii) If $\left(\tau_{l}, \tau_{k}\right)=\left(\tau_{l}, b\right)$ is a boundary cluster at b, then (37) holds.

Clearly, Theorem 5 follows from Lemma 7.1, and Theorems 6 and 7 .

To prove Theorem 7, we need some preparation.

10.1. The calculation of $a_{11}$. In subsequent sections we will compute the expression $\mathbf{P} \Psi\left(\tau_{i+1}, \tau_{i-1}\right) \mathbf{P}^{-1}$. From this, we need to estimate $a_{i j}(i, j=1,2)$. Especially, for interior spikes $\left(\tau_{l}, \tau_{k}\right)$, we find that the sign of $w$ at $\tau_{l+2 m}$ is determined by $a_{11}$, which will be shown to relate to $\dot{S}$. The following lemmas provide this information. Their proofs, however, will be given in the Appendix. 
Lemma 10.1. If $J(x) \neq 0$, then

$$
\begin{gathered}
\frac{d}{d x} S(x)=\int_{\phi_{0}}^{\phi^{*}} \frac{F_{x x}(s, x)}{\sqrt{2 F(s, x)}} d s-\int_{\phi_{0}}^{\phi} \frac{F_{x}^{2}(s, x)}{(2 F(s, x))^{3 / 2}} d s-\int_{\phi}^{\phi^{*}} \frac{F_{x}^{2}(s, x)-F_{x}^{2}\left(\phi^{*}, x\right)}{(2 F(s, x))^{3 / 2}} d s \\
-\frac{F_{x}^{2}\left(\phi^{*}, x\right)}{f\left(\phi^{*}, x\right)}\left\{\frac{1}{\sqrt{2 F(\phi, x)}}-\int_{\phi}^{\phi^{*}} \frac{f(s, x)-f\left(\phi^{*}, x\right)}{(2 F(s, x))^{3 / 2}} d s\right\}
\end{gathered}
$$

where the argument for $\phi_{0}, \phi^{*}$ and $\phi$ is $x$.

Lemma 10.2. Assume that $f_{i} \propto 1, F_{i}=O\left(\varepsilon^{2}|\ln \varepsilon|\right)$, and $f_{i \pm 1}^{2}=O\left(\varepsilon^{2}|\ln \varepsilon|\right)$. Then

$$
\left(\frac{\varepsilon^{2} \dot{u}_{i+1 / 2}^{2} \varepsilon^{2} \dot{u}_{i-1 / 2}^{2}}{f_{i}^{2}}\right) a_{11}=2 \mathrm{H}\left(J\left(\tau_{i}\right)\right) \dot{S}\left(\tau_{i}\right)+O(\varepsilon|\ln \varepsilon|) .
$$

Lemma 10.3. Assume that $f_{i} \propto 1$ and $f_{i \pm 1}^{2}=O(\varepsilon)$. Then $a_{11}=O(1)$.

10.2. First approximation for $\mathbf{P} \Psi\left(\tau_{i+1}, \tau_{i-1}\right) \mathbf{P}^{-1}$. For spikes, we can use the following approximation for fundamental solution matrices. In this lemma and subsequently, $r$ denotes a positive number, not necessarily the same at each occurrence.

Lemma 10.4. Assume that $f_{i}^{2} \propto 1$ and $f_{i \pm 1}^{2}=O(\varepsilon)$. Then for some positive $r$

$$
\begin{aligned}
& \mathbf{P} \Psi\left(\tau_{i}, \tau_{i-1}\right) \mathbf{P}^{-1}=-r\left\{\left[\begin{array}{l}
0 \\
1
\end{array}\right]\left[\begin{array}{ll}
\alpha_{i-1} & 1
\end{array}\right]+O(\sqrt{\varepsilon})\right\}, \\
& \mathbf{P} \Psi\left(\tau_{i+1}, \tau_{i}\right) \mathbf{P}^{-1}=-r\left\{\left[\begin{array}{c}
1 \\
\alpha_{i+1}
\end{array}\right]\left[\begin{array}{ll}
1 & 0
\end{array}\right]+O(\sqrt{\varepsilon})\right\} .
\end{aligned}
$$

If in addition we have $\frac{\varepsilon^{2}|\ln \varepsilon|}{f_{i-1}^{2}+f_{i+1}^{2}}=O(1)$, then

$$
\mathbf{P} \Psi\left(\tau_{i+1}, \tau_{i-1}\right) \mathbf{P}^{-1}=r\left\{\left[\begin{array}{c}
1 \\
\alpha_{i+1}
\end{array}\right]\left[\begin{array}{ll}
1 & 0
\end{array}\right] \mu+\hat{\mu}\left[\begin{array}{l}
0 \\
1
\end{array}\right]\left[\begin{array}{ll}
\alpha_{i-1} & 1
\end{array}\right]+O\left(\frac{1}{\mid \ln \varepsilon}\right)\right\},
$$

where $(\mu, \hat{\mu})=\left(1, \frac{f_{i+1}^{2}}{f_{i-1}^{2}}\right)$ if $\frac{f_{i+1}^{2}}{f_{i-1}^{2}}=O(1)$ and $(\mu, \hat{\mu})=\left(\frac{f_{i-1}^{2}}{f_{i+1}^{2}}, 1\right)$ if $\frac{f_{i-1}^{2}}{f_{i+1}^{2}}=O(1)$.

Proof. We use (29) and (30). The estimates for $\mathbf{t}_{R+}^{\perp}$ and $\mathbf{t}_{L-}$ are the same as for layers, since $f_{i \pm 1}^{2}=O(\varepsilon)$. For $\mathbf{P} \Psi\left(\tau_{i+1}, \tau_{i}\right) \mathbf{P}^{-1}$, we see from (31) that $\frac{\varepsilon}{\omega_{+}^{2}}=$ $O\left(\frac{f_{i+1}^{2}}{\varepsilon}\right)=O(1)$ and $\frac{\varepsilon}{\omega_{-}^{2}} \propto \frac{f_{i}^{2}}{\varepsilon} \propto \frac{1}{\varepsilon}$. Hence, the term involving $\frac{\varepsilon}{\omega_{-}^{2}}$ is the dominant term, and we have (43). The assertion (42) is similarly derived.

The assertion (44) follows from (30) by estimating the coefficients $a_{i j}$. For example, for $a_{12}$ since all the jumps $\llbracket \rho \rrbracket, \llbracket \gamma \rrbracket$ are $O(1)$, and

$$
\omega_{L-}=\frac{\varepsilon^{2} \dot{u}_{i-1 / 2}}{f_{i-1}} c\left(z_{i-1 / 2}-0\right) \propto \frac{\varepsilon}{f_{i-1}}, \quad \omega_{R-}=\frac{\varepsilon^{2} \dot{u}_{i+1 / 2}}{f_{i}} c\left(z_{i-1 / 2}+0\right) \propto \frac{\varepsilon}{f_{i}},
$$

we have

$$
a_{12}=\frac{\varepsilon^{2}}{\omega_{L-}^{2} \omega_{R-}^{2}}\left(1+O\left(\frac{\omega_{R-}^{2}}{\varepsilon}\right)\right)=\frac{\varepsilon^{2}}{\omega_{L-}^{2} \omega_{R-}^{2}}(1+O(\varepsilon)) \propto \frac{f_{i-1}^{2} f_{i}^{2}}{\varepsilon^{2}} \propto \frac{f_{i-1}^{2}}{\varepsilon^{2}} .
$$

Hence, if $\tau_{i-1} \neq \tau_{l}$, use the estimates for $F_{i-1}$ in Section 4 and the relation $f_{i-1}^{2} \propto$ $F_{i-1}$, to yield that either $f_{i-1}^{2} \propto \varepsilon$ or $f_{i-1}^{2} \propto \varepsilon^{2}|\ln \varepsilon|$. We then have $a_{12} \geq C|\ln \varepsilon|$. A similar estimate holds for $a_{21}$ if $\tau_{i+1} \neq \tau_{k}$. Since by Lemma $10.3 a_{11}=O(1)$, we see that $a_{12}$ and $a_{21}$ are the dominant terms, which leads to (44). 
10.3. Boundary spikes at $a$. We first consider the case when $\left(\tau_{0}, \tau_{k}\right)$ is an interval with a boundary, at $a$, cluster of spikes. We consider two cases: (i) $k$ is odd, (ii) $k$ is even.

Suppose that $k$ is even. Then $\tau_{2 m+1}$ for $m=0, \cdots, k / 2-1$ are peaks of the spikes. Also, $\frac{f_{2 m+2}^{2}}{f_{2 m}^{2}}=O(1)$ for $m=0, \cdots, k / 2-1$. Hence, using $w(a)=1, \dot{w}(a)=0$ and (44) we obtain

$$
\tilde{\mathbf{w}}\left(\tau_{2 m}\right)=\left|w\left(\tau_{2 m}\right)\right|\left[\begin{array}{c}
1 \\
\nu_{2 m}+o(1)
\end{array}\right], \quad m=0,1, \cdots, k / 2,
$$

where $\nu_{2 m}$ are iteratively defined by

$$
\nu_{0}=0, \quad \nu_{2 m+2}=\alpha_{2 m+2}+\frac{f_{2 m+2}^{2}}{f_{2 m}^{2}}\left(\alpha_{2 m}+\nu_{2 m}\right) .
$$

In particular, since $f_{k}=O\left(e^{-1 / \sqrt{\varepsilon}}\right)$, we have $\nu_{k}=\alpha_{k}+o(1)$. Thus, $w\left(\tau_{2 m}\right)>0$ for each $m=1, \cdots, k / 2$. This implies that $w=0$ has exactly two roots in each $\left(\tau_{2 m}, \tau_{2 m+2}\right)$. Hence, $w=0$ has $k$ roots in $\left(a, \tau_{k}\right)$, and (36) holds.

Next suppose that $k$ is odd. Then $\tau_{0}=a$ is a peak. Using $w(a)=1, \dot{w}(a)=0$ and (43) we see that $\tilde{\mathbf{w}}\left(\tau_{1}\right)=-\left|w\left(\tau_{1}\right)\right|\left[\begin{array}{c}1 \\ \alpha_{1}+o(1)\end{array}\right]$. This implies that $w=0$ has exactly one root in $\left(a, \tau_{1}\right)$. From $\tau_{1}$ to $\tau_{k}$, we follow the same argument as before to conclude that $w=0$ has exactly $k$ roots in $\left(a, \tau_{k}\right)$, and (36) holds.

10.4. Boundary spikes at $b$. Next we consider the case when $\left(\tau_{l}, \tau_{n}\right)$ is an interval with a boundary, at $b$, cluster of spikes. We have, by assumption, that $w\left(\tau_{l}\right) \dot{w}\left(\tau_{l}\right) \geq$ 0 . We want to show that (37) holds. By working with $-w$ if necessary, we can assume that $w\left(\tau_{l}\right) \geq 0$ and $\dot{w}\left(\tau_{l}\right) \geq 0$.

First we show that $(-1)^{i-l} \dot{w}\left(\tau_{i}\right)>0$ for all $i=l+1, \cdots, n$. We note that $f_{l}=O\left(e^{-1 / \sqrt{\varepsilon}}\right)$ and $\frac{f_{l+2 m}^{2}}{f_{l+2 m+2}^{2}}=O(1)$ for all $m=0, \cdots,\left[\frac{n-l-2}{2}\right]$. Hence, using (42) and (44), we inductively derive that $\tilde{\mathbf{w}}\left(\tau_{i}\right)=(-1)^{i-l}\left|\varepsilon \dot{w}\left(\tau_{i}\right)\right|\left[\begin{array}{c}o(1) \\ 1\end{array}\right]$ for all $i=l+1, \cdots, k$. Therefore, $(-1)^{i-l} \dot{w}\left(\tau_{i}\right)>0$ for all $i=l+1, \cdots, n$. In particular, $\dot{w}(b) \neq 0$.

To find the number of zeros of $w$ in $\left(\tau_{l}, b\right)$, we will compare the roots of $w=0$ with the roots of $\bar{w}=0$, where $\bar{w}$ solves

$$
\varepsilon^{2} \ddot{\bar{w}}=f_{u} \bar{w} \quad \text { in } \quad(a, b), \quad \bar{w}(b)=1, \quad \dot{\bar{w}}(b)=0 .
$$

By reversing the $x$ axes and using the argument for boundary spikes at $a$ in the previous subsection, we see that $\bar{w}=0$ has exactly $n-l$ roots in $\left(\tau_{l}, b\right)$. Hence, by the Liouville theorem, the number of roots of $w=0$ in $\left(\tau_{l}, b\right)$ is at least $n-l-1$ and at most $n-l+1$.

Now suppose that $w(b) \dot{w}(b)>0$. Then $(-1)^{n-l} w(b)>0$. It then follows that the number of roots in $\left(\tau_{l}, b\right)$ is $(n-l) \bmod (2)$. Thus, the number of roots is exactly $n-l$.

Next we consider the case $w(b) \dot{w}(b) \leq 0$. Then, by the Liouville theorem, one derives that the right-most root to $\bar{w}=0$ in $\left(\tau_{l}, b\right]$ is on the right-hand side of the right-most root of $w=0$ in $\left(\tau_{l}, b\right)$. Hence, the number of roots of $w=0$ in $\left(\tau_{l}, b\right)$ is at most $n-l$. Noting that either $(-1)^{n-l-1} w(b)>0$ or $w(b)=0$ and $(-1)^{n-l} \dot{w}(b)<0$, we conclude from the sign change of $w$ that the number of roots of $w=0$ in $\left(\tau_{l}, b\right)$ is $(n-l-1) \bmod (2)$. Hence, the number of roots of $w=0$ in $\left(\tau_{l}, b\right)$ is $n-l-1$. This completes the proof of (37). 
10.5. Interior spikes. Finally, we consider interior spikes. Hence, assume that $\left(\tau_{l}, \tau_{k}\right)$ is an interval with an interior cluster of spikes. Then $k-l$ is even, $f_{l+2 m}=$ $O\left(\varepsilon^{2}|\ln \varepsilon|\right)$ for $m=0, \cdots,(k-l) / 2$ and $f_{l+2 m+1} \propto 1$ for $m=0, \cdots,(k-l) / 2-1$.

Without loss of generality, we assume that $w\left(\tau_{l}\right) \geq 0$ and $\dot{w}\left(\tau_{l}\right) \geq 0$.

First suppose that we have a single spike. Then both $f_{l}$ and $f_{l+2}$ are of size $O\left(e^{-1 / \sqrt{\varepsilon}}\right)$, so that $\frac{1}{\omega_{L-}^{2}}, \frac{1}{\omega_{R+}^{2}}=O\left(e^{-1 / \sqrt{\varepsilon}}\right)$. It then follows that, for some $r>0$,

$$
\mathbf{P} \Psi\left(\tau_{l+2}, \tau_{l}\right) \mathbf{P}^{-1}=r\left\{a_{11}\left[\begin{array}{c}
1 \\
\alpha_{l+2}+o(\varepsilon)
\end{array}\right]\left[\begin{array}{ll}
\alpha_{l}+O(\varepsilon) & 1
\end{array}\right]+O\left(e^{-c / \sqrt{\varepsilon}}\right)\right\} .
$$

Since $a_{11} \propto \mathrm{H}\left(J\left(z^{*}\right)\right) \dot{S}\left(z^{*}\right)$, we then conclude that, for $k=l+2$,

$$
\tilde{\mathbf{w}}\left(\tau_{k}\right)=(-1)^{s}\left|w\left(\tau_{k}\right)\right|\left[\begin{array}{c}
1 \\
\alpha_{k}+o(1)
\end{array}\right] \mathrm{H}\left(w_{l}+\dot{w}_{l}\right),
$$

where $s=1$ if $J\left(z^{*}\right) \dot{S}\left(z^{*}\right)<0$ and $s=2$ if $J\left(z^{*}\right) \dot{S}\left(z^{*}\right)>0$. It follows that (36) holds. In the latter case, we conclude that $w=0$ has two roots in $\left(\tau_{l}, \tau_{l+2}\right)$. In the former case we know that there are either 1 or 3 roots, and we must show that there is only one.

For this purpose, we first calculate, from (42),

$$
\begin{aligned}
\tilde{\mathbf{w}}\left(\tau_{l+1}\right) & =\mathbf{P} \Psi\left(\tau_{l+1}, \tau_{l}\right) \mathbf{P}^{-1} \tilde{\mathbf{w}}\left(\tau_{l}\right)=-r\left\{\left[\begin{array}{cc}
0 & 0 \\
\alpha_{l} & 1
\end{array}\right]+O(\sqrt{\varepsilon})\right\}\left[\begin{array}{c}
w_{l} \\
\varepsilon \dot{w}_{l}
\end{array}\right] \\
& =-H\left(w_{l}+\varepsilon \dot{w}_{l}\right)\left|\varepsilon \dot{w}_{l+1}\right|\left[\begin{array}{c}
O(\sqrt{\varepsilon}) \\
1
\end{array}\right]
\end{aligned}
$$

and so

$$
\frac{\varepsilon \dot{w}_{l+1}}{w_{l+1}}=O(\sqrt{\varepsilon}) \text {. }
$$

Then, a technique similar to one used in Section 9.4 shows that $w_{l} w_{l+1 / 2} \geq 0$ and $w_{l+3 / 2} w_{l+2}>0$. It thus follows that $w=0$ has exactly one root in $\left(z_{l}, z_{l+2}\right]$.

Next we consider the case $k-l>2$. Then we must have $J\left(z^{*}\right) \dot{S}\left(z^{*}\right)>0$ so that $a_{11}>0$ and is of order 1 . Since $f_{l+2 m}^{2}=O\left(\varepsilon^{2}|\ln \varepsilon|\right)$, we see that $\frac{\varepsilon}{\omega_{L-}^{2}}, \frac{\varepsilon}{\omega_{R+}^{2}}=$ $O(\varepsilon|\ln \varepsilon|)$. Also, $\frac{\varepsilon}{\omega_{L+}^{2}}, \frac{\varepsilon}{\omega_{R-}^{2}} \propto \frac{1}{\varepsilon}$. Therefore, all the entries in the $2 \times 2$ matrix $\mathbf{P} \Psi\left(\tau_{l+2 m}, \tau_{l+2 m-2}\right) \mathbf{P}^{-1}$ are positive. This implies that $w\left(\tau_{2 m}\right)>0$ and $\dot{w}\left(\tau_{2 m}\right)>0$ for all $m=1, \cdots, k / 2$, and $w=0$ has two roots in $\left(\tau_{2 m-2}, \tau_{2 m}\right)$. Consequently, $w=0$ has $k-l$ roots in $\left(\tau_{l}, \tau_{k}\right)$.

Note in particular that for the last matrix $\mathbf{P} \Psi\left(\tau_{k}, \tau_{k-2}\right) \mathbf{P}^{-1}$, the quantities $a_{21}$ and $a_{22}$ are exponentially small so that

$$
\mathbf{P} \Psi\left(\tau_{k-2}, \tau_{k}\right) \mathbf{P}^{-1}=r\left\{\mathbf{t}_{R}^{\perp} \otimes\left(a_{11} \mathbf{t}+a_{12} \mathbf{e}_{1}\right)+O\left(e^{-c / \sqrt{\varepsilon}}\right)\right\} .
$$

It then follows that (36) holds. This completes the proof.

\section{An EXtension}

In this section, we extend our theory to (47) $f(u, x)=A(u, x)\left[u-\phi_{-}(x)\right][u-\phi(x)]\left[u-\phi_{+}(x)\right], \quad A>0, \phi_{-}<\phi<\phi_{+}$.

By a calculus of variation, for all small positive $\varepsilon>0$, (1) admits a unique minimal solution $u_{-}(x, \varepsilon)$ and a unique maximal solution $u_{+}(x, \varepsilon)$ which satisfy

$$
\left\|u_{ \pm}-\phi_{ \pm}\right\|_{C^{0}}+\varepsilon\left\|u_{ \pm}-\phi_{ \pm}\right\|_{C^{1}}+\varepsilon^{2}\left\|u_{ \pm}\right\|_{C^{2}}=O\left(\varepsilon^{2}\right) .
$$


Introduce the change of variables $(u, x) \rightarrow(\theta, y)$ by

$$
y=y_{\varepsilon}(x):=\int_{a}^{x} \frac{d z}{\left(u_{+}(z, \varepsilon)-u_{-}(z, \varepsilon)\right)^{2}}, \quad \theta=\frac{u-u_{-}}{u_{+}-u_{-}} .
$$

Then problem (11) for $u(x)$ is equivalent to the new problem, for $\theta(y)$,

$$
\varepsilon^{2} \theta^{\prime \prime}(y)=f_{\varepsilon}(\theta, y) \text { in }\left(0, b_{\varepsilon}\right), \quad \theta^{\prime}(0)=\theta^{\prime}\left(b_{\varepsilon}\right)=0,
$$

where ${ }^{\prime}=\frac{d}{d y}, b_{\varepsilon}=y_{\varepsilon}(b)$, and

$$
f_{\varepsilon}(\theta, y)=\left(u_{+}-u_{-}\right)^{3}\left\{f\left(\theta u_{+}+(1-\theta) u_{-}, x\right)-(1-\theta) f\left(u_{-}, x\right)-\theta f\left(u_{+}, x\right)\right\} .
$$

Since $u_{ \pm}=\phi_{ \pm}+O\left(\varepsilon^{2}\right), f\left(u^{ \pm}, x\right)=O\left(\varepsilon^{2}\right)$. It then follows that for all small positive $\varepsilon, f_{\varepsilon}(\cdot, y)=0$ has exactly three roots: $0, \phi_{\varepsilon}, 1$, where $\lim _{\varepsilon \backslash 0} \phi_{\varepsilon}=\frac{\phi-\phi_{-}}{\phi_{+-} \phi_{-}}$.

For $f_{\varepsilon}$, denote the corresponding functions defined in Section 1 by $J_{\varepsilon}, F_{\varepsilon}$ and $S_{\varepsilon}$. For $f$, we define

$$
\begin{aligned}
& J(x)=\int_{\phi_{-}(x)}^{\phi_{+}(x)} f(s, x) d s, \quad \phi_{0}(x)= \begin{cases}\phi_{+}(x) & \text { if } J(x) \geq 0, \\
\phi_{-}(x) & \text { if } J(x)<0,\end{cases} \\
& F(u, x)=\int_{\phi_{0}(x)}^{u} f(s, x) d s, \quad \phi^{*}(x) \in\left[\phi_{-}, \phi_{+}\right] \backslash\left\{\phi_{0}\right\}: F\left(\phi^{*}, x\right)=0, \\
& S(x)=\frac{d}{d x} \int_{\phi_{0}(x)}^{\phi^{*}(x)} \sqrt{2 F(s, x)} d x=\int_{\phi_{0}}^{\phi^{*}} \frac{F_{x}(s, x)}{\sqrt{2 F(s, x)}} d s \text { if } J(x) \neq 0 .
\end{aligned}
$$

Using (48), one can directly verify, as $\varepsilon \searrow 0$, the following limits in the $C^{1}$ topology:

$$
\begin{aligned}
f_{\varepsilon}(\theta, y) & \longrightarrow\left(\phi_{+}-\phi_{-}\right)^{3} f\left(\theta \phi_{+}+(1-\theta) \phi_{-}, x\right), \\
J_{\varepsilon}(y) & \longrightarrow J_{0}(y):=\left(\phi_{+}-\phi_{-}\right)^{2} J(x), \\
S_{\varepsilon}(y) & \longrightarrow S_{0}(y):=\left(\phi_{+}-\phi_{-}\right)^{2} S(x) \text { when } J(x) \neq 0,
\end{aligned}
$$

where $x$ and $y$ are related by

$$
y=\int_{a}^{x} \frac{d z}{\left(\phi_{+}(z)-\phi_{-}(z)\right)^{2}} .
$$

Note that $J_{0}(y)=0 \Leftrightarrow J(x)=0$ and $S_{0}(y)=0 \Leftrightarrow S(x)=0$. In addition,

$$
\begin{aligned}
& J^{2}+\dot{J}^{2}>0 \Leftrightarrow J_{0}^{2}+J_{0}^{\prime 2}>0 \Rightarrow \exists \varepsilon_{0}, \eta>0 \quad \ni \quad J_{\varepsilon}^{2}+J_{\varepsilon}^{\prime 2} \geq \eta \forall \varepsilon \in\left(0, \varepsilon_{0}\right], \\
& S^{2}+\dot{S}^{2}>0 \Leftrightarrow S_{0}^{2}+S_{0}^{\prime 2}>0 \Rightarrow \exists \varepsilon_{0}, \eta>0 \quad \ni \quad S_{\varepsilon}^{2}+S_{\varepsilon}^{\prime 2} \geq \eta \forall \varepsilon \in\left(0, \varepsilon_{0}\right] .
\end{aligned}
$$

These properties ensure that our results in the previous sections apply to (49). Since $Z_{J_{\varepsilon}}$ and $Z_{S_{\varepsilon}}$ are in an $O(\varepsilon)$ neighborhood of $Z_{J_{0}}$ and $Z_{S_{0}}$ respectively, we see that the conclusion for solutions to (49) can be stated with $Z_{J_{\varepsilon}}$ and $Z_{S_{\varepsilon}}$ being substituted by $Z_{J_{0}}$ and $Z_{S_{0}}$. Transferring back to the $x$ variable, we obtain the corresponding results; in particular, layers exist only near $Z_{J}$ and spikes only near $Z_{S}$ with $J$ and $S$ defined by (50).

Finally, we provide an example where

$$
f(u, x)=u^{3}-\lambda u+p(x) .
$$

It is easy to see that $f$ can be put into the form (47) provided that

$$
\lambda>3\left(\frac{1}{2}\|p\|_{L^{\infty}}\right)^{2 / 3} .
$$


In such a case, one can calculate, by (50), that

$$
J(x) \propto p(x), \quad S(x) \propto \dot{p}(x)(\text { when } p(x) \neq 0) .
$$

Thus, layers exist near the zeros of $p$, interior multiple spikes exist near nondegenerate negative maxima or positive minima of $p$, and there exist only interior single spikes near non-degenerate positive maxima and negative minima of $p$.

When $p(x)=\cos x$, these results suggest what was, in fact, proved in $[\mathrm{AH}$, namely that solutions exist with layers near odd multiples of $\frac{\pi}{2}$ and single spikes at multiples of $\pi$. There do not exist interior multiple spikes. The methods of this paper also serve to determine the Morse index of these solutions.

\section{APPENDIX}

Proof of Lemma 10.1. Since $F(s, x)$ vanishes quadratically around $s=\phi_{0}$ and linearly around $s=\phi^{*}$, all the integrals involved are convergent. Also, differentiating $F\left(\phi^{*}, x\right)=0$ gives us $F_{x}\left(\phi^{*}, x\right)+\phi_{x}^{*} f\left(\phi^{*}, x\right)=0$. For any fixed constant $a$ between $\phi_{0}$ and $\phi^{*}$, we can write

$$
S(x)=\int_{\phi_{0}}^{a} \frac{F_{x}}{\sqrt{2 F}} d s+\int_{a}^{\phi^{*}} \frac{F_{x}+f \phi_{x}^{*}}{\sqrt{2 F}} d s+\phi_{x}^{*} \sqrt{2 F(a, x)} .
$$

A direct differentiation then gives

$$
\begin{aligned}
\frac{d}{d x} S(x)= & \int_{\phi_{0}}^{\phi^{*}} \frac{F_{x x}}{\sqrt{2 F}} d s-\int_{\phi_{0}}^{a} \frac{F_{x}^{2}}{(2 F)^{3 / 2}} d s-\int_{a}^{\phi^{*}} \frac{\left(F_{x}+f \phi_{x}^{*}\right) F_{x}}{(2 F)^{3 / 2}} d s \\
& +\int_{a}^{\phi^{*}} \frac{f \phi_{x x}^{*}+f_{x} \phi_{x}^{*}}{\sqrt{2 F}} d s+\phi_{x x}^{*} \sqrt{2 F(a, x)}+\frac{\phi_{x}^{*} F_{x}(a, x)}{\sqrt{2 F(a, x)}} .
\end{aligned}
$$

Note that

$$
\begin{aligned}
\int_{a}^{\phi^{*}} \frac{f \phi_{x x}^{*}+f_{x} \phi_{x}^{*}}{\sqrt{2 F}} d s=- & \phi_{x x}^{*} \sqrt{2 F(a, x)}-\phi_{x}^{*} \frac{F_{x}(a, x)-F_{x}\left(\phi^{*}, x\right)}{\sqrt{2 F(a, x)}} \\
& +\int_{a}^{\phi^{*}} \frac{\phi_{x}^{*}\left(F_{x}-F_{x}\left(\phi^{*}, x\right)\right) f}{(2 F)^{3 / 2}} d s
\end{aligned}
$$

Thus,

$\frac{d}{d x} S(x)=\int_{\phi_{0}}^{\phi^{*}} \frac{F_{x x}}{\sqrt{2 F}} d s-\int_{\phi_{0}}^{a} \frac{F_{x}^{2}}{(2 F)^{3 / 2}} d s-\int_{a}^{\phi^{*}} \frac{F_{x}^{2}+f F_{x}\left(\phi^{*}, x\right) \phi_{x}^{*}}{(2 F)^{3 / 2}} d s+\frac{\phi_{x}^{*} F_{x}\left(\phi^{*}, x\right)}{\sqrt{2 F(a, x)}}$.

Here each unstated argument of $F_{x}, F_{x x}$, or $f$ is $(s, x)$. The lemma thus follows by replacing $\phi_{x}^{*}=-F_{x}\left(\phi^{*}, x\right) / f\left(\phi^{*}, x\right)$ and setting $a=\phi$.

Proof of Lemma 10.2. Without loss of generality, we assume that $\tau_{i}$ is a local maximum, so that $f_{i}<0, J\left(\tau_{i}\right)<0, \dot{u}_{i+1 / 2}<0$ and $\dot{u}_{i-1 / 2}>0$.

First, we calculate $\llbracket \rho \rrbracket_{R-}^{R+}=\llbracket \rho \rrbracket_{z_{i+1 / 2}-0}^{z_{i+1 / 2}+0}$. From (31), we see that $\rho=\frac{\dot{c}}{c}$ at $z_{i+1 / 2}$. Using $G(u, v, t)=F(u, t)-F(v, t)$, we have $-G_{x}\left(u, u_{i}, x\right)+\int_{\tau_{i}}^{x} G_{x x}\left(u, u_{i}, t\right) d t=$ $-F_{x}(u, x)+F_{x}\left(u_{i}, \tau_{i}\right)+\int_{\tau_{i}}^{x} F_{x x} d t$ and $-G\left(u, u_{i+1}, x\right)+\int_{\tau_{i+1}}^{x} G_{x x}\left(u, u_{i+1}, t\right) d t=$ 
$-F_{x}(u, x)+F_{x}\left(u_{i+1}, \tau_{i+1}\right)+\int_{\tau_{i+1}}^{x} F_{x x} d t$. Hence, from (32), we have

$$
\begin{aligned}
\varepsilon^{2} \dot{u}_{i+1 / 2}^{2} \llbracket \rho \rrbracket_{z_{i+1 / 2}-0}^{z_{i+1 / 2}+0}= & F_{x}\left(u_{i+1}, \tau_{i+1}\right)-F_{x}\left(u_{i}, \tau_{i}\right)+\int_{\tau_{i+1}}^{\tau_{i}} F_{x x}(u(t), t) d t \\
& -\int_{\tau_{i+1}}^{z_{i+1 / 2}} \frac{G_{x}^{2}\left(u, u_{i+1}, t\right)}{\varepsilon^{2} \dot{u}^{2}} d t-\int_{z_{i+1 / 2}}^{\tau_{i}} \frac{G_{x}^{2}\left(u, u_{i}, t\right)}{\varepsilon^{2} \dot{u}^{2}} d t+O\left(\varepsilon^{2}\right) .
\end{aligned}
$$

Note that $F_{x}\left(u_{i+1}, \tau_{i+1}\right)=O\left(f_{i+1}^{2}\right)=O\left(\varepsilon^{2}|\ln \varepsilon|\right)$. Also, denoting $F_{x}^{*}=F_{x}\left(\phi^{*}\left(\tau_{i}\right), \tau_{i}\right)$, then $F_{x}\left(u_{i}, \tau_{i}\right)=F_{x}^{*}+O\left(\varepsilon^{2}|\ln \varepsilon|\right)$ since $F$ vanishes linearly at $\phi^{*}$ and $F_{i}=$ $O\left(\varepsilon^{2}|\ln \varepsilon|\right)$ implies that $\phi^{*}\left(\tau_{i}\right)-u_{i}=O\left(\varepsilon^{2}|\ln \varepsilon|\right)$. Thus, using a technique similar to that used in the proof of Lemma 4.1 we then obtain

$$
\begin{aligned}
\varepsilon^{2} \dot{u}_{i+1 / 2}^{2} \llbracket \rho \rrbracket_{z_{i+1 / 2}-0}^{z_{i+1 / 2}+0}=- & F_{x}^{*}-\varepsilon \int_{0}^{\phi_{i}}\left(\frac{F_{x x}}{\sqrt{2 F}}-\frac{F_{x}^{2}}{(2 F)^{3 / 2}}\right) d s \\
& \quad-\varepsilon \int_{\phi_{i}}^{\phi_{i}^{*}}\left(\frac{F_{x x}}{\sqrt{2 F}}-\frac{\left(F_{x}-F_{x}^{*}\right)^{2}}{(2 F)^{3 / 2}}\right) d s+O\left(\varepsilon^{2}|\ln \varepsilon|\right) .
\end{aligned}
$$

Here and below each unstated argument of $F, F_{x}, F_{x x}$, or $f$ is $\left(s, \tau_{i}\right)$.

Note that we integrate $\frac{\dot{c}}{c}$ in (32) and use a technique similar to that used in showing (17) to get

$$
c\left(z_{i+1 / 2}-0\right)=1+\varepsilon c_{1}, \quad c_{1}=-\int_{\phi_{i}}^{\phi_{i}^{*}} \frac{F_{x}-F_{x}^{*}}{(2 F)^{3 / 2}} d s+O(\varepsilon|\ln \varepsilon|) .
$$

Then using $\omega_{R-}^{2}=\varepsilon^{2} \dot{u}_{i+1 / 2}^{2} \frac{\varepsilon^{2}}{f_{i}^{2}} c^{2}\left(z_{i+1 / 2}-0\right)$ we obtain

$$
\begin{aligned}
& \frac{f_{i}^{2} \omega_{R-}^{2}}{\varepsilon^{2}} \llbracket \rho \rrbracket_{R-}^{R+}=\left(1+2 \varepsilon c_{1}+\varepsilon^{2} c_{1}^{2}\right) \varepsilon^{2} \dot{u}_{i+1 / 2}^{2} \llbracket \rho \rrbracket_{z_{i+1 / 2}-0}^{z_{i+1 / 2+0}} \\
& =-F_{x}^{*}-\varepsilon \int_{0}^{\phi_{i}}\left(\frac{F_{x x}}{\sqrt{2 F}}-\frac{F_{x}^{2}}{(2 F)^{3 / 2}}\right) d s-\varepsilon \int_{\phi_{i}}^{\phi_{i}^{*}}\left(\frac{F_{x x}}{\sqrt{2 F}}-\frac{\left(F_{x}-F_{x}^{*}\right)^{2}}{(2 F)^{3 / 2}}\right) d s \\
& \quad+2 \varepsilon F_{x}^{*} \int_{\phi_{i}}^{\phi_{i}^{*}} \frac{F_{x}-F_{x}^{*}}{(2 F)^{3 / 2}} d x+O\left(\varepsilon^{2}|\ln \varepsilon|\right) \\
& =-F_{x}^{*}+\varepsilon \mathrm{H}\left(\dot{u}_{i+1 / 2}\right)\left\{\int_{\phi_{0}}^{\phi_{i}}\left(\frac{F_{x x}}{\sqrt{2 F}}-\frac{F_{x}^{2}}{(2 F)^{3 / 2}}\right) d s+\int_{\phi_{i}}^{\phi_{i}^{*}}\left(\frac{F_{x x}}{\sqrt{2 F}}-\frac{F_{x}^{2}-F_{x}^{* 2}}{(2 F)^{3} / 2}\right) d s\right\} \\
& \quad+O\left(\varepsilon^{2}|\ln \varepsilon|\right) .
\end{aligned}
$$

Similarly, we can calculate $\omega_{L+}^{2} \llbracket \rho \rrbracket_{L+}^{L-}=-\omega_{L+}^{2} \llbracket \rho \rrbracket_{L-}^{L+}$ and obtain the same expression. As $\mathrm{H}\left(\dot{u}_{i+1 / 2}\right)=-\mathrm{H}\left(\dot{u}_{i-1 / 2}\right)=\mathrm{H}\left(f_{i}\right)$, we then obtain

$$
\begin{aligned}
& \frac{f_{i}^{2}}{\varepsilon^{2}}\left\{\omega_{L+}^{2} \llbracket \rho \rrbracket_{L-}^{L+}+\omega_{R-}^{2} \llbracket \rho \rrbracket_{R-}^{R+}\right\} \\
& =2 \varepsilon \mathrm{H}\left(f_{i}\right)\left\{\int_{\phi_{0}}^{\phi_{i}}\left(\frac{F_{x x}}{\sqrt{2 F}}-\frac{F_{x}^{2}}{(2 F)^{3 / 2}}\right) d s+\int_{\phi_{i}}^{\phi_{i}^{*}}\left(\frac{F_{x x}}{\sqrt{2 F}}-\frac{F_{x}^{2}-F_{x}^{* 2}}{(2 F)^{3} / 2}\right) d s\right\} \\
& \quad+O\left(\varepsilon^{2}|\ln \varepsilon|\right) .
\end{aligned}
$$

Note that

$$
\omega_{L+}^{2} \llbracket \rho \rrbracket_{L-}^{L+}=\frac{\varepsilon^{2}}{f_{i}^{2}}\left\{F_{x}^{*}+O(\varepsilon)\right\}, \quad \omega_{R-}^{2} \llbracket \rho \rrbracket_{R-}^{R+}=\frac{\varepsilon^{2}}{f_{i}^{2}}\left\{-F_{x}^{*}+O(\varepsilon)\right\} .
$$


Also, by Lemma 8.2, we have

$$
\llbracket \gamma \rrbracket_{L+}^{R-}=2 f_{i} \mathrm{H}\left(f_{i}\right)\left\{\frac{1}{\sqrt{2 F\left(\phi_{i}, \tau_{i}\right)}}-\int_{\phi_{i}}^{\phi_{i}^{*}} \frac{f-f^{*}}{(2 F)^{3 / 2}} d s\right\}+O(\varepsilon|\ln \varepsilon|) .
$$

It then follows that

$$
\begin{aligned}
& \omega_{L+}^{2} \omega_{R-}^{2} a_{11}=\varepsilon \omega_{L+}^{2} \llbracket \rho \rrbracket_{L-}^{L+}+\varepsilon \omega_{R-}^{2} \llbracket \rho \rrbracket_{R-}^{R+}+\omega_{L+}^{2} \llbracket \rho \rrbracket_{L-}^{L+} \llbracket \gamma \rrbracket_{L+}^{R-} \omega_{R-}^{2} \llbracket \rho \rrbracket_{R-}^{R+} \\
= & \frac{2 \varepsilon^{4} \mathrm{H}\left(f_{i}\right)}{f_{i}^{2}}\left\{\int_{\phi_{0}}^{\phi}\left(\frac{F_{x x}}{\sqrt{2 F}}-\frac{F_{x}^{2}}{(2 F)^{3 / 2}}\right) d s+\int_{\phi_{i}}^{\phi_{i}^{*}}\left(\frac{F_{x x}}{\sqrt{2 F}}-\frac{F_{x}^{2}-F_{x}^{* 2}}{(2 F)^{3} / 2}\right) d s\right. \\
& \left.\quad-\frac{F_{x}^{* 2}}{f_{i}}\left\{\frac{1}{\sqrt{2 F\left(\phi_{i}, \tau_{i}\right)}}-\int_{\phi_{i}}^{\phi_{i}^{*}} \frac{f-f^{*}}{(2 F)^{3 / 2}} d s\right\}+O(\varepsilon|\ln \varepsilon|)\right\} \\
= & \frac{2 \varepsilon^{4} \mathrm{H}\left(f_{i}\right)}{f_{i}^{2}}\left\{\dot{S}\left(\tau_{i}\right)+O(\varepsilon|\ln \varepsilon|)\right\} .
\end{aligned}
$$

Finally, using

$$
\omega_{L+}^{2} \omega_{R-}^{2}=\frac{\varepsilon^{8} \dot{u}_{i+1 / 2}^{2} \dot{u}_{i-1 / 2}^{2}}{f_{i}^{4}}[1+O(\varepsilon)],
$$

we then obtain the assertion of the lemma.

Proof of Lemma 10.3. The proof follows the same lines as in the proof of the previous lemma, with the simplification that all the integrals for the coefficient of $\varepsilon$ be replaced by $O(1)$. In particular, $F_{x}\left(u_{i \pm 1}, \tau_{i \pm 1}\right)=O\left(f_{i \pm 1}^{2}\right)=O(\varepsilon)$. We omit the details.

\section{REFERENCES}

[A] S. Ai, A bvp on a singularly perturbed second order ode by Carrier, J. Math. Anal. Appl. 277 (2003), 405-422. MR1961235 (2004b:34043)

[AH] S. Ai and S. P. Hastings, A shooting approach to layers and chaos in a forced duffing equation, J. Diff. Eqns. 185 (2002), 389-436. MR.1935609 (2003i:70028)

[ABF] N. D. Alikakos, P. W. Bates, G. Fusco, Solutions to the nonautonomous bistable equation with specified Morse index. Part I. Existence, Trans. Amer. Math. Soc. 340 (1993) 641654. MR:1167183 (94b:34032)

[AMPP] S. Angenent, J. Mallet-Paret and L. A. Peletier, Stable transition layers in a semilinear boundary value problem, J. Diff. Eqns. 67 (1987), 212-242. MR0879694 (88d:34018)

[CP] J. Carr and B. Pego, Metastable patterns in solution of $u_{t}=\varepsilon^{2} u_{x x}+f(u)$, Comm. Pure Appl. Math. 42 (1989), 523-576. MR0997567 (90f:35091)

[FR1] B. Fiedler and C. Rocha, Heteroclinic orbits of semilinear parabolic equations, J. Diff. Eqns. 125 (1996), 239-281. MR.1376067 (96k:58200)

[FR2] B. Fiedler and C. Rocha, Orbit equivalence of global attractors of semilinear parabolic differential equations, Trans. AMS 35 (2000), 252-284. MR.1475682(2000c:35129)

[HASL] J. K. Hale and J. D. Salazar, Boundary layers in a semilinear parabolic problem, Tohoku Math J. (2) 51 (1999), 421-432. MR1707765(2000i:35010)

[HSG] J. K. Hale and J. D. Salazar-Gonzalez, Attractors of some reaction diffusion problems, SIAM J. Math Anal. 30 (1999), 963-984. MR.1709783 (2000g:35108)

[HS] J. K. Hale and K. Sakamoto, Existence and stability of transition layers, Japan Journal of Applied Mathematics 5 (1988), 367-405. MR0965871 (90a:35112)

[HM] S. P. Hastings and J. B. Mcleod, On the periodic solutions of a forced second-order equation, J. Nonlinear Sci. 1 (1991), 225-245. MR1118986 (93e:34060a)

[KU] H. Kurland, Monotone and oscillatory equilibrium solutions of a problem arising in population genetics, Contemp. Math., 17, Amer. Math. Soc., Providence, R.I., 1983, pp. 323-342. MR0706107 (84i:92050) 
[N] K. Nakashima, Multi-layered stationary solutions for a spatially inhomogeneous AllenCahn equation, J. Diff. Eqns. 191 (2003), 234-276. MR.1973289 (2004f:35097)

[RO] C. Rocha, On the singular problem for the scalar parabolic equation with variable diffusion, J. Math. Anal. Appl. 183 (1994), 413-428. MR1274148(95b:35118)

Department of Mathematical Sciences, University of Alabama at Huntsville, Huntsville, Alabama 35899

E-mail address: ais@email.uah.edu

Department of Mathematics, University of Pittsburgh, Pittsburgh, Pennsylvania 15260

E-mail address: xinfu@pitt.edu

Department of Mathematics, University of Pittsburgh, Pittsburgh, Pennsylvania 15260

E-mail address: sph@pitt.edu 